\title{
NMDQi Thermal-Hydraulic Optimization, Analysis, and Scoping Tool (TOAST)
}

\section{December 2020}

Abdullah G. Weiss

Idaho National Laboratory

Department of Nuclear Engineering, Texas A\&M University

Austen D. Fradeneck

Idaho National Laboratory

Geoffrey L. Beausoleil II

Idaho National Laboratory

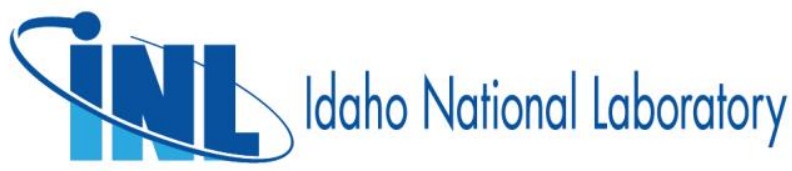




\section{DISCLAIMER}

This information was prepared as an account of work sponsored by an agency of the U.S. Government. Neither the U.S. Government nor any agency thereof, nor any of their employees, makes any warranty, expressed or implied, or assumes any legal liability or responsibility for the accuracy, completeness, or usefulness, of any information, apparatus, product, or process disclosed, or represents that its use would not infringe privately owned rights. References herein to any specific commercial product, process, or service by trade name, trademark, manufacturer, or otherwise, does not necessarily constitute or imply its endorsement, recommendation, or favoring by the U.S. Government or any agency thereof. The views and opinions of authors expressed herein do not necessarily state or reflect those of the U.S. Government or any agency thereof. 
INL/EXT-20-60711

Revision 3

\title{
NMDQi Thermal-Hydraulic Optimization, Analysis, and Scoping Tool (TOAST)
}

\author{
Abdullah G. Weiss \\ Idaho National Laboratory \\ Department of Nuclear Engineering, Texas A\&M University \\ Austen D. Fradeneck \\ Idaho National Laboratory \\ Geoffrey L. Beausoleil II \\ Idaho National Laboratory
}

December 2020

Idaho National Laboratory

Idaho Falls, Idaho 83415

http://www.inl.gov

Prepared for the

U.S. Department of Energy

Office of Nuclear Energy

Under DOE Idaho Operations Office

Contract DE-AC07-05ID14517 
Page intentionally left blank 


\begin{abstract}
The Thermal-hydraulic Optimization, Analysis, and Scoping Tool (TOAST) is developed to support the thermal-hydraulic aspects for the design of optimal irradiation vehicles for the Nuclear Materials Discovery and Qualification initiative (NMDQi). TOAST is currently a MATLAB-based tool that utilizes a simplified steady-state analytical model where a radial thermal resistance circuit is utilized to account for conduction through up to 2 capsules, a gas gap, a thermal bond, a sample cladding, and a sample, as well as the convection from a water coolant outside the capsules. The geometry is discretized axially along the user-defined height of a basket with a user-defined number of points/nodes, essentially turning TOAST into a semi-2D heat transfer analysis utility for cylindrical irradiation vehicles with practically any configuration, solving for temperatures radially at multiple axial locations. TOAST utilizes a Graphical User Interface (GUI) in-which a user can define the geometrical layout, the materials utilized for each component, the coolant characteristics, and the required solution. The user can define the geometry by inputting the diameters, thicknesses, and heights for each component, whereas the materials are defined via the user-provided constant or variable thermal conductivities. The user can select the coolant's inlet temperature, pressure, and the pressure drop across the height of the problem (which is used to calculate the velocity of the flow). Finally, the user can choose to solve for a sample heat generation rate limit by inputting temperature limits for the sample and capsules or can choose to purely solve for the axial temperature distributions of each component by inputting a pre-selected heat generation rate. Either way, TOAST provides the user with axial temperature distributions of all the components including the coolant, and the heat generation rate limit as well as the maximum outlet coolant temperature. The user can also choose to do one of 6 sensitivity analyses in TOAST. The sensitivity analyses yield plots of the sensitivity of the heat generation rate limit, the maximum coolant temperature, and the pressure limit for the annular components due to perturbations in 1-2 unknown variables based on the selected sensitivity analysis. Benchmarks between computations in TOAST and equivalent $2 \mathrm{D}$ axis symmetric ABAQUS models are presented, showing that TOAST results are within less than $3 \%$ of ABAQUS results in most cases, and a maximum of $8 \%$ difference in some cases. The benchmarks also revealed that this uncertainty is tied to the selection of an appropriate Nusselt number correlation and appropriate thermal conductivities, which the user can do from the GUI. Regardless, TOAST is demonstrated as a computationally efficient, highly accessible, and accurate utility for optimization and scoping $\mathrm{s}$ of studies different irradiation vehicles.
\end{abstract}


Page intentionally left blank 


\section{CONTENTS}

ABSTRACT iii

ACRONYMS viii

1. PREFACE

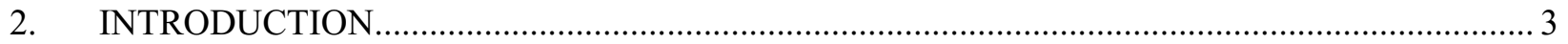

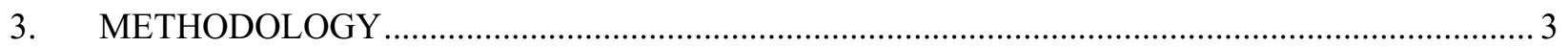

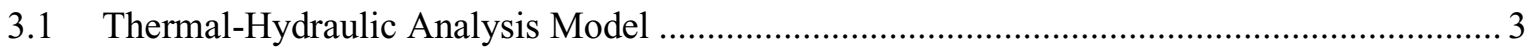

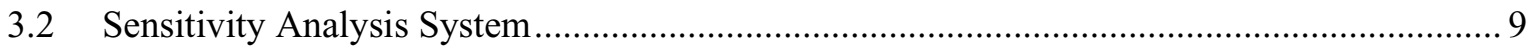

3.2.1 Sensitivity analysis with respect to the basket ID ( $\mathrm{Dbi}$........................................ 11

3.2.2 Sensitivity analysis with respect to the OD of capsule 1 (Dc1) ............................... 12

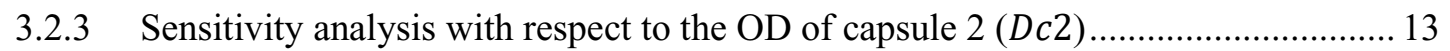

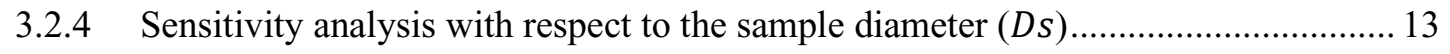

3.2.5 Sensitivity analysis with respect to the bond thickness (tbond) with a set sample temperature $(\mathrm{Ts}$, limit $)$...................................................................... 14

3.2.6 Sensitivity analysis with respect to the bond thickness (tbond) with a varied sample temperature limit (Ts, limit) ................................................................. 15

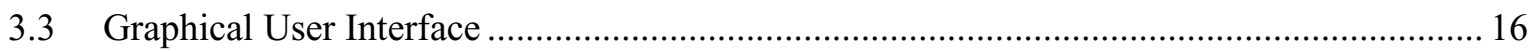

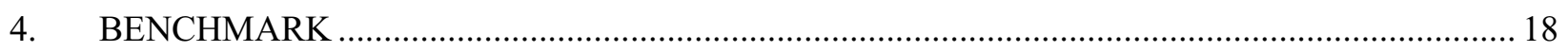

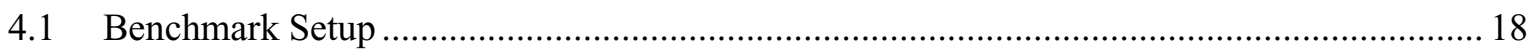

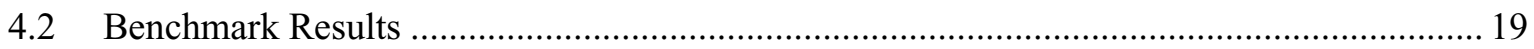

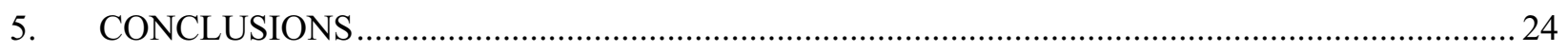

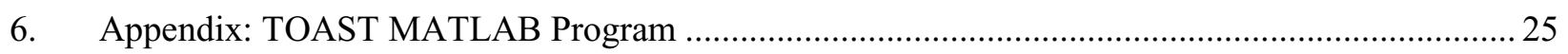

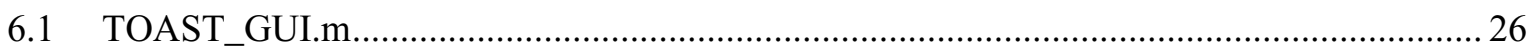

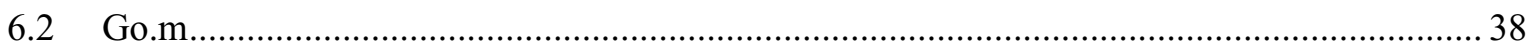

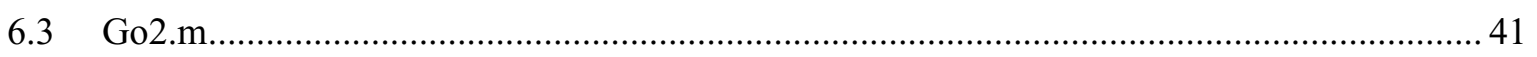

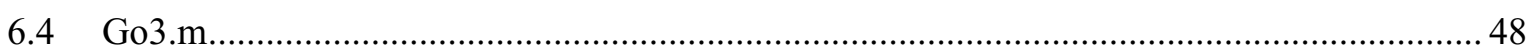

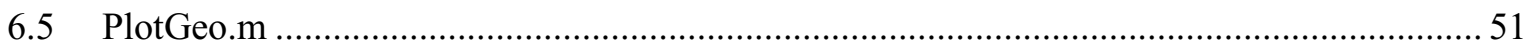

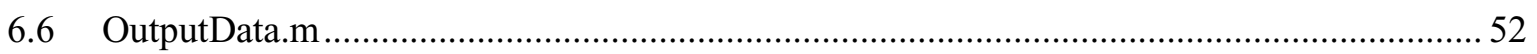

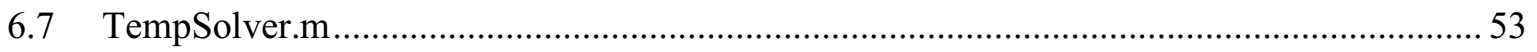

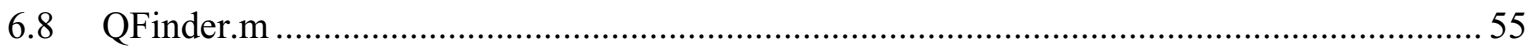

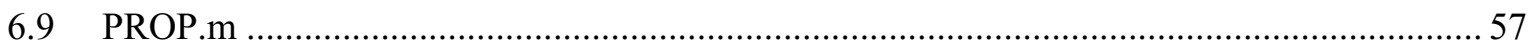

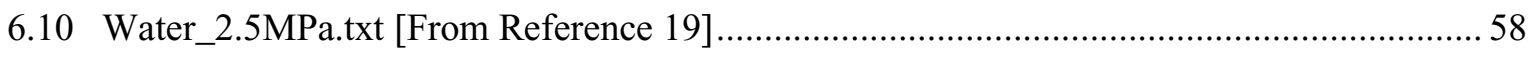

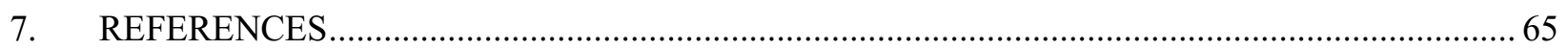




\section{FIGURES}

Figure 1. Nuclear reactor generations and a proposed timeline [2] .............................................. 1

Figure 2. Percent share of every energy source in the U.S. throughout the years [3] ............................. 2

Figure 3. A top view of the generalized layout setup for TOAST. ......................................................... 4

Figure 4. A side view of the simplified geometrical setup for TOAST ................................................. 4

Figure 5. The radial thermal resistance circuit used for each axial position modeled in TOAST............... 5

Figure 6. $K f$ evolution as a function of $R e$ using Churchill's equation for the friction factor $f$............... 9

Figure 7. The automated procedure for the sensitivity analysis with respect to $\mathrm{Dbi}$.............................. 12

Figure 8 . The automated procedure for the sensitivity analysis with respect to $D c 1$........................... 12

Figure 9. The automated procedure for the sensitivity analysis with respect to $D c 2$. ............................. 13

Figure 10. The automated procedure for the sensitivity analysis with respect to $D s$............................. 14

Figure 11. The automated procedure for the sensitivity analysis with respect to tbond........................... 14

Figure 12. The automated procedure for the sensitivity analysis with respect to tbond and a

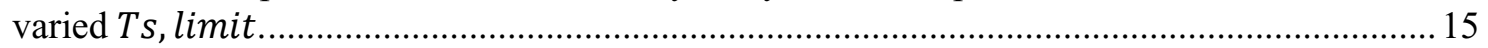

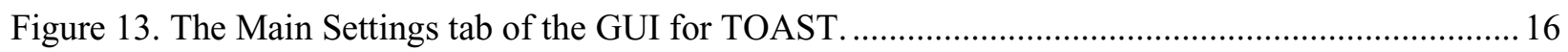

Figure 14.The Additional Settings tab of the GUI for TOAST. .............................................................. 17

\section{TABLES}

Table 1. Constant conductivity benchmarks.

Table 2. Percent difference between ABAQUS and TOAST for the constant conductivity benchmarks.

Table 3. Variable conductivity benchmarks.

Table 4. Percent difference between ABAQUS and TOAST for the variable conductivity benchmarks. 
Page intentionally left blank 


\section{ACRONYMS}

AFC

AFC-FAST

ANS

ASME

ATF

ATR

DNBR

FIR

GUI

INL

MOOSE

NMDQi

NRC

ODs

IDs

THAT

TOAST

$\mathrm{V} \& \mathrm{~V}$
Advanced Fuel Campaign

Advanced Fuels Campaign Fission Accelerated Steady-State Testing

American Nuclear Society

American Society of Mechanical Engineers

accident tolerant fuel

Advanced Test Reactor

departure from nucleate boiling ratio

flow instability ratio

graphical user interface

Idaho National Laboratory

Multiphysics Object-Oriented Simulation Environment

Nuclear Materials Discovery and Qualification initiative

Nuclear Regulatory Commission

outer diameters

inner diameters

Thermal-Hydraulic Analysis Team

Thermal-hydraulic Optimization, Analysis, and Scoping Tool

verification and validation 
Page intentionally left blank 


\section{NMDQi Thermal-Hydraulic Optimization, Analysis, and Scoping Tool (TOAST)}

\section{PREFACE}

In winter 2016, Dr. Andrew Klein, then president of the American Nuclear Society (ANS), launched a nuclear grand challenge effort, wherein more than 125 nuclear professionals and students conducted roundtable brainstorming sessions to come up with ideas for such challenges that must be resolved by 2030 [1]. By June 2017, all the ideas were consolidated into the following nine grand challenges via recommendations made by more than 300 professionals in multiple ANS divisions.

1. "Transform the way the nuclear technologies sector thinks about public engagement."

2. "Establish the scientific basis for modern low-dose radiation regulation."

3. "Close the nuclear fuel cycle."

4. "Ensure continuous availability of radioisotopes."

5. "Rejuvenate nuclear technology infrastructure and facilities."

6. "Accelerate development and qualification of advanced materials."

7. "Accelerate utilization of simulation and experimentation."

8. "Expedite licensing and deployment of advanced reactor designs."

9. "Expedite nuclear education updates and knowledge transfer."

It is important to note that the general reoccurring theme across most of the challenges is the need for establishing tools and capabilities to accelerate and expedite the development of new technologies and rejuvenate the existing nuclear technology infrastructure. The nuclear industry is potentially the slowest industry at adapting to changes and technological advancements, as is evidenced by the lengthy and costly licensing and qualification processes for anything related to the design and construction of a nuclear reactor. Other industries and markets react to technological advancements much differently, which is clearly demonstrated in the vast differences between an iPhone 4 (2010) and an iPhone 11 (2019) (per information found on www.gadgetsnow.com).

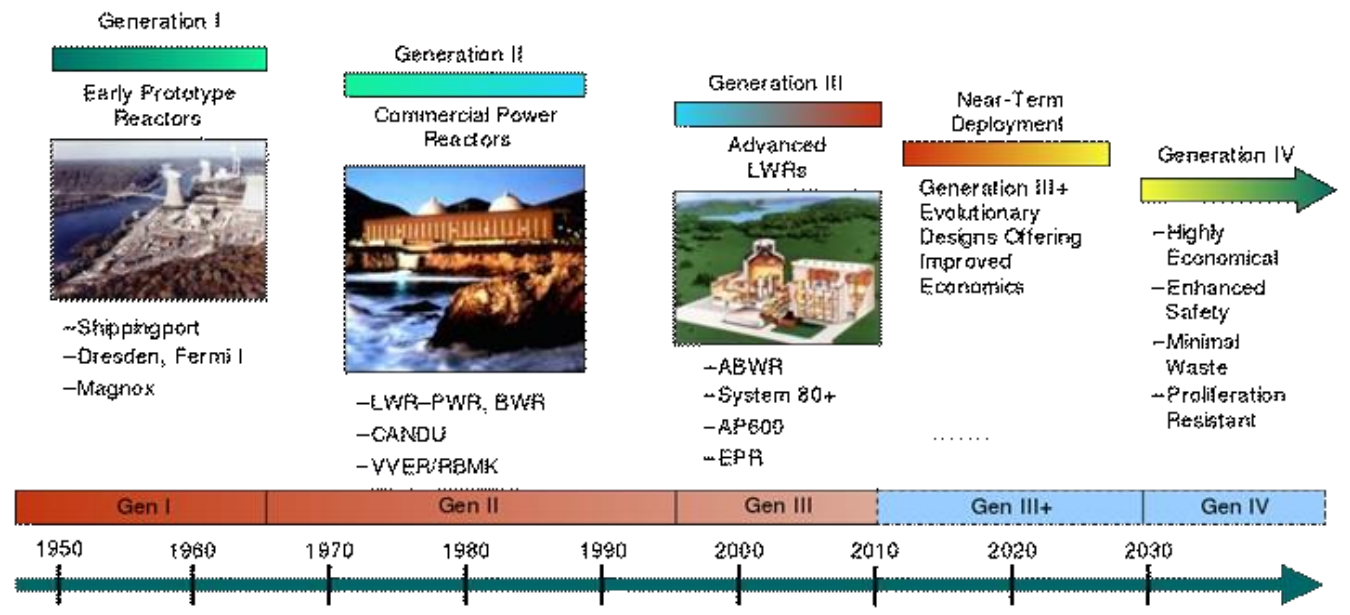

Figure 1. Nuclear reactor generations and a proposed timeline [2]. 
In this decade, the iPhone processor speed more than doubled and the RAM storage more than quadrupled all-while the launch price (accounting for inflation) remained approximately the same. In that same decade, advances in new nuclear infrastructure (reactors, powerplants) exponentially grew without many changes to the existing technology. The nuclear industry sported commercial nuclear reactors from Gen II (PWRs, BWRs, CANDUs) for decades without showing much intergenerational progress. It wasn't until the recent opening of the Barakah reactor unit in Abu Dhabi, UAE that a reactor from a "new generation" has surfaced in the market. The sluggishness and costliness in nuclear technological advancements is shown in the generic reactor generations chart (see Figure 1), where Gen III reactors seem like necessary safety upgrades to Gen II reactors and an intermediary generation was necessary to separate Gen III and the elusive Gen IV with, once again, the evolutions of Gen II reactors.

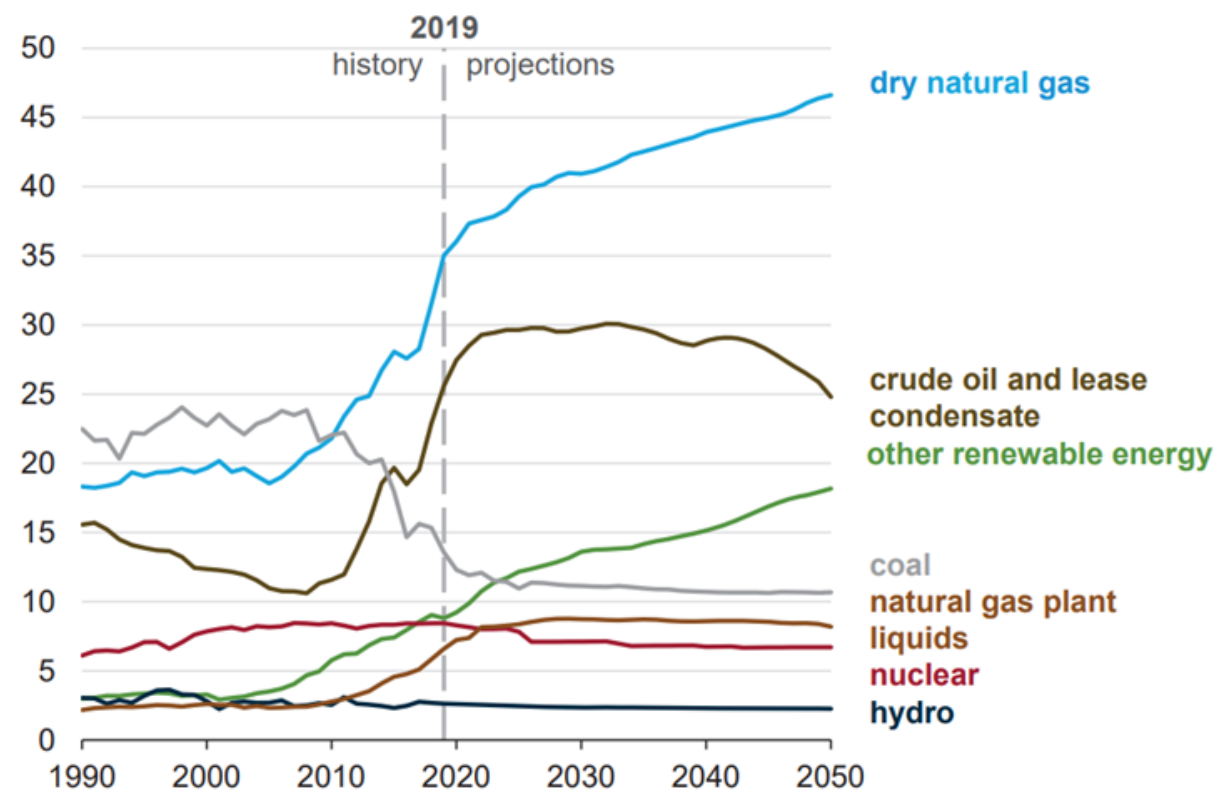

Figure 2. Percent share of every energy source in the U.S. throughout the years [3].

While natural gas, crude oil, and renewable energy production shares increased within this decade, nuclear almost flat-lined, with projections of its energy share decreasing as renewable energy subsidies increase. The gap left by decreases in the massive nuclear power capacity cannot be solely replaced by renewables, forcing an increased reliance on volatile natural gas, which in turn would contribute to a larger natural gas energy share. This increased natural gas reliance may be economical in the short-term, but long-term disadvantages would far outweigh those short-term economic advantages. For starters, an increase in natural gas energy production would lead to increased fracking and the production of more greenhouse gases, further contributing to global warming. Additionally, as the reliance on natural gas grows so will its demand, meaning that sooner-or-later the supply will suffer and the prices will increase, potentially offsetting any short-term economic advantages. For those reasons, it is important to keep nuclear power competitive in the energy market with advanced nuclear reactors that are more efficient and less costly than existing technologies. This is the primary goal of most of the listed nuclear challenges.

Multiple initiatives have since been developed to address those challenges, with efforts primarily directed to the verification and validation $(\mathrm{V} \& \mathrm{~V})$ of thermal-hydraulic computational fluid dynamics and system-level codes (Nuclear Regulatory Commission initiatives [4], American Society of Mechanical Engineers (ASME) V\&V Problems), and, of most interest to this report, an accelerated nuclear materials discovery and qualification process. To support all those initiatives, it is vital to develop more accessible tools, correlations, methodologies, and databases to accelerate and refine the current processes. 


\section{INTRODUCTION}

Due to the nature of the nuclear materials discovery and qualification process, multiple irradiation experiments are necessary to assist in qualification efforts. This leads to large arrays of iterative tests with various irradiation conditions. Naturally, this implies that each experiment conducted would consist of a unique and specific combination of dimensions, materials, incident neutron flux, and heat loads. Each experiment's characteristics are typically chosen using a set of computational studies prior to irradiation to ensure appropriate experiments are carried out safely $[5,6]$. This procedure implies multidecade development cycles to iterate through the variations of a material (fuel, clad, structural support, etc.) in order to qualify it $[7,8]$. Not only does this suggest extremely high financial and labor costs towards the qualification of advance nuclear materials, it also makes it less likely for innovative materials to be considered as the discovery process of such materials will further extend the development procedure. This suppresses innovation in the field of nuclear materials, as lengthy development processes come with an increased financial burden and decreased likelihood for implementation during our lifetimes, driving away potential investors and aspiring scientists and engineers. As such, it is no surprise that future nuclear technologies will require a shortened and more streamlined development and deployment timeline, which implies a shortened discovery and qualification process for nuclear materials [9].

To support this sentiment, the Nuclear Materials Discovery and Qualification Initiative (NMDQi) was recently established as an effort to develop the tools and capabilities for supporting the accelerated discovery and engineering qualification of new materials for use in advanced nuclear reactors [10,11]. The acceleration of thermal-hydraulic optimization studies for irradiation experiments, particularly at the Advanced Test Reactor (ATR) at Idaho National Laboratory (INL) [12], is of particular interest to the work outlined in this report, where a versatile thermal analysis tool is developed with sensitivity and parametric analysis capabilities for different experiment dimensions (fuel, clad, capsules, etc.). This tool serves as a dimensional and thermal-hydraulic optimization utility that can drive decisions about the sizing of any material in an irradiation environment with any set of thermal-hydraulic characteristics (temperature limits, coolant flow rates, linear heat generation rates, etc.) while maintaining computational efficiency (i.e., little computational power is required).

This report outlines the methodology by which the thermal-hydraulic analysis model is driven, how the sensitivity analysis system works, and the frameworks that allows the user to interface with the models and its results. Benchmark problems are also presented to provide an understanding of the tool's uncertainties and their sources as compared to results from similar models in the commercial software ABAQUS [13], which is typically used in thermal-hydraulic safety and programmatic analyses at INL.

\section{METHODOLOGY}

\subsection{Thermal-Hydraulic Analysis Model}

The Thermal-Hydraulic Optimization, Analysis, and Scoping Tool (TOAST) adopts a generalized geometry layout that closely reflects the Advanced Fuel Campaign (AFC) irradiation vehicle layout $[5,14]$ in an effort to remain computationally inexpensive and highly accessible. This general layout allows for a wide range of cylindrical irradiation vehicles, accommodating single-capsule and multicapsule setups. This geometrical layout is illustrated in Figure 3, where a basket contains the coolant channel (water for the purposes of this report). Within the coolant channel lies an outer capsule (Capsule 1) casing an inner capsule (Capsule 2) with a gas gap in between the two capsules. Capsule 2 holds a thermal bond of some thickness separating the capsule from the rodlet clad, which houses the sample. 


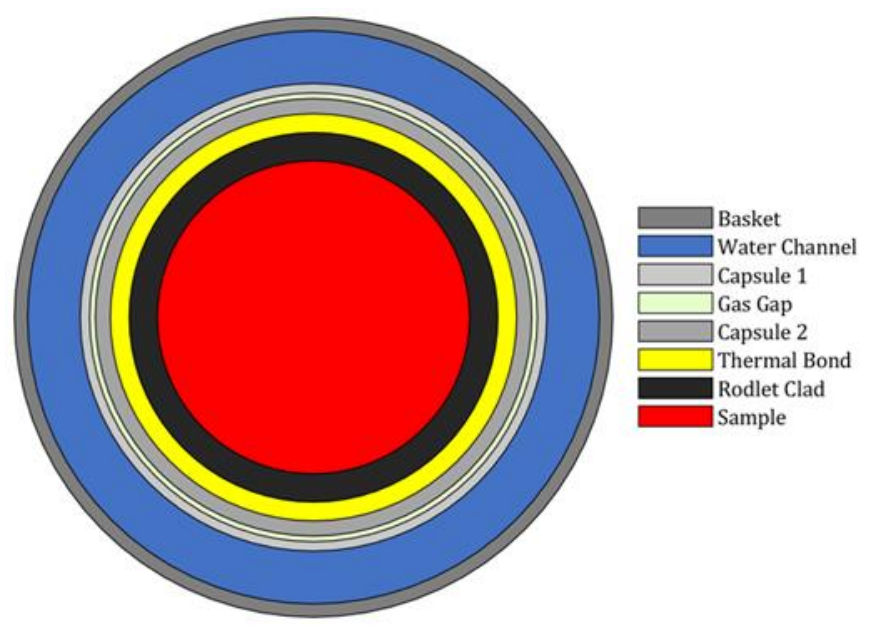

Figure 3. A top view of the generalized layout setup for TOAST.

The layout above is simplified by assuming symmetry in the axial and radial thermal profiles, yielding the simplified geometrical setup shown in Figure 4, where only a quarter of the geometry is found necessary with the aforementioned assumptions. It is implied that the user has control over the dimensions and thermal conductivities of the components in the irradiation vehicle (capsules, clad, and sample) as well as the flow rate (proxy of the pressure drop) and inlet temperature and pressure of the coolant. Note that end-caps are not accounted for in this model, but the actual coolant path is maintained. As such, unlike what Figure 4 suggests, no flow occurs directly above the sample and clad but instead occurs directly above the capsules.

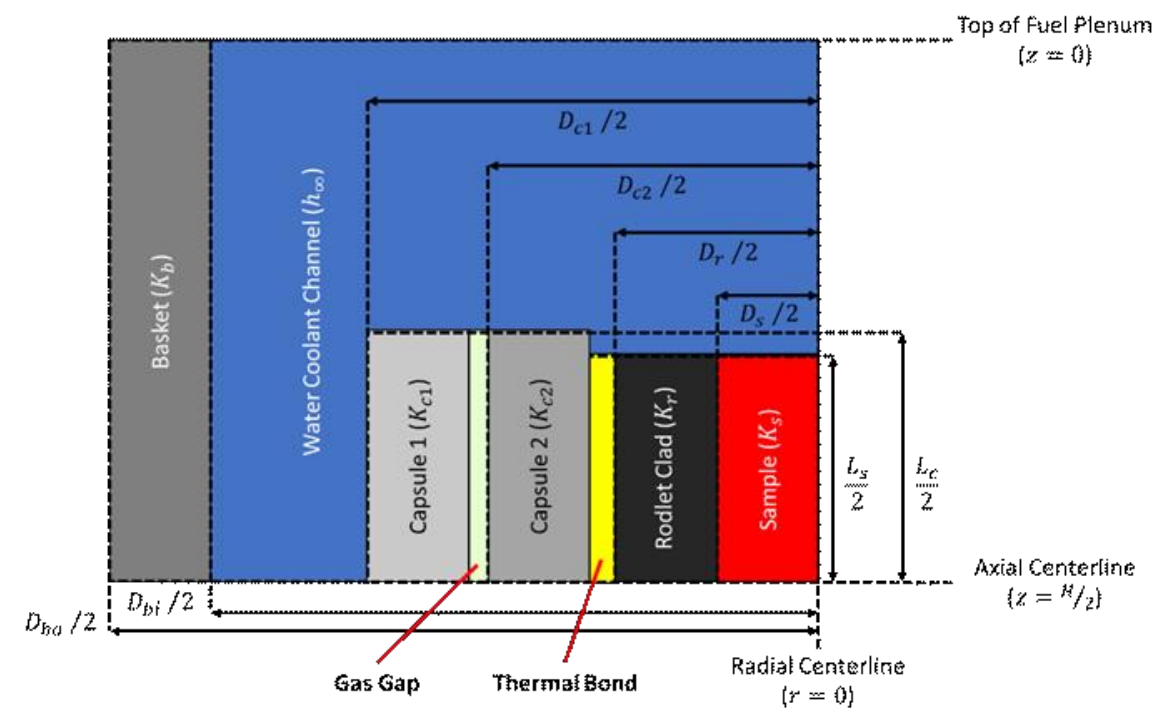

Figure 4. A side view of the simplified geometrical setup for TOAST. 
With the geometrical layout defined, an appropriate thermal analysis model can be developed. The model developed for TOAST is a semi-2D model that provides axial temperature distributions at specific radial positions by solving a radial heat transfer network at multiple axial locations. The selected radial positions are at the inner surface of each of the components and at the sample centerline, due to the assumption that the highest temperature for each component would be at its innermost radial position. As such, the radial positions are listed below, and they consist of the inner diameters (IDs) and outer diameters (ODs) of different components.

- Coolant temperature at the innermost location (OD of Capsule 1 when the capsule is reached or at the radial centerline otherwise)

- ID of Capsule 1

- $\quad$ ID of Capsule 2

- ID of the rodlet clad

- Radial centerline of the sample

The axial (height-wise) direction is uniformly discretized via a set of equally spaced points (e.g., nodes) spanning the entire height of the geometry (typically the height of the basket). The number of axial points is selected by the user in TOAST. In a sense, this setup corresponds to a $2 \mathrm{D}$ mesh that is discretized uniformly in the axial direction with a user-set number of points and exactly five points in the radial direction. In that sense, the mesh is very coarse compared to meshes that are generated in other finite element or volume codes, with thousands of nodes in all directions. It somewhat corresponds to modeling approaches implemented in system-level thermal-hydraulic codes, such as RELAP [15] and TRACE [16], or severe accident codes, such as MELCOR [17]. This radial coarseness in the spatial discretization is beneficial, as it allows for the inexpensive computation of the components' maximum temperatures, which is usually of high importance for scoping and optimization studies. This is possible by only considering the innermost points of each of the components, where the data predicts the maximum temperatures. A finer radial discretization is possible in a future version of TOAST; however, for the time-being, this simplified approach is more advantageous.

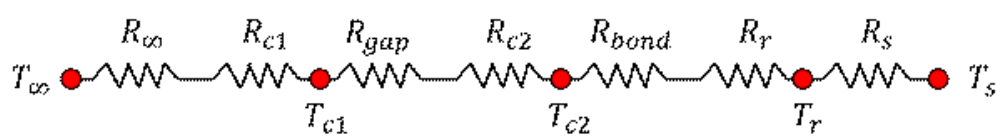

Figure 5. The radial thermal resistance circuit used for each axial position modeled in TOAST.

Corresponding to the simplified geometrical setup shown in Figure 4 and the aforementioned discretization system, a thermal resistance circuit can be setup for different axial positions to model the steady-state temperature profile at each radial position throughout the geometry. This leads to the circuit shown in Figure 5, where the coolant temperature $\left(T_{\infty}\right)$ is used to calculate the inner temperature of Capsule $1\left(T_{c 1}\right)$ using the thermal resistance due to convection through the coolant $\left(R_{\infty}\right)$ and conduction through Capsule $1\left(R_{c 1}\right) . T_{c 1}$ can then be used to calculate the inner temperature of Capsule $2\left(T_{c 2}\right)$ by accounting for the thermal resistance due to conduction through the gas gap $\left(R_{g a p}\right)$ and Capsule $2\left(R_{c 2}\right)$. Similarly, $T_{c 2}$ is used to calculate the rodlet clad inner temperature $\left(T_{r}\right)$ by accounting for thermal resistances due to conduction through the thermal bond $\left(R_{\text {bond }}\right)$ and through the rodlet clad $\left(R_{r}\right)$. Finally, the sample centerline temperature $\left(T_{s}\right)$ can be calculated using $T_{r}$ and the thermal resistance due to conduction through the sample $\left(R_{S}\right)$. Although the in-depth description of the circuit is seemingly unnecessary due to its intuitiveness, we will define the working variables of the problem when discussing the thermal resistance circuit and will further elaborate in later sections of this document. 
$T_{\infty}$ is calculated by considering the heat rate of the sample and assuming the coolant inhibits a cosine heating distribution of a similar format to that of the chopped cosine axial gamma heat distribution at different irradiation experiment positions in ATR [18]. The cosine heating distribution for the water is shown in Equation 1, where the sample heat rate $\left(q^{\prime}{ }_{\text {limit }}\right)$, spacing between axial discretization points $(\Delta z)$, the sample length $\left(L_{s}\right)$ are accounted for alongside a cosine fitting constant ( $c$, assumed to be 1 for this report), the axial position relative to the top of the basket $(z)$, the axial centerline relative to the top of the basket $\left(z_{\text {cent }}\right)$, and the maximum heat rate offset from the axial centerline $\left(x_{0}\right)$.

$q^{\prime}(z)=q_{\text {limit }}^{\prime} \Delta z \cos \left(c\left[\left|z-z_{\text {cent }}\right|-x_{0}\right]\right)$

The axial distribution of the coolant temperature can then be derived assuming a constant mass flow rate $(\dot{m})$ and specific heat $\left(c_{p}\right)$, yielding the discretization-friendly relation shown in Equation 2 . In Equation 2, the coolant temperature at each axial position $(z)$ is calculated by adding the sample heating contributions to the coolant temperature at the previous axial location $(z-1)$, such that $z=0$ represents the inlet boundary of the flow with a user-specified inlet temperature.

$T_{\infty}(z)=T_{\infty}(z-1)+\left(\frac{q^{\prime}{ }_{\text {limit }} \Delta z}{\dot{m} c_{p}} \sin \left(c\left[\left|z-z_{\text {cent }}\right|-x_{0}\right]\right)+\sin \left(c\left[z_{\text {cent }}-x_{0}\right]\right)\right)$

As for the $q_{\text {limit }}^{\prime}$, which drives the whole thermal analysis procedure, it is important to reiterate that the user has the choice to provide the sample linear heat generation rate $\left(q_{s}^{\prime}\right)$ or provide the temperature limits for the capsules and the sample. If the user provides $q_{s}^{\prime}, q^{\prime}$ limit is calculated using Equation $3 \mathrm{a}$ based on the user's preference for a constant or a cosine-shaped heat-rate profile in the axial direction. If the user provides temperature limits, three different heat rates are calculated, corresponding to each temperature limit, and the minimum of the three is the $q_{\text {limit }}^{\prime}$, as shown in Equation $3 \mathrm{~b} . T_{\infty, \max }$ corresponds to the maximum coolant temperature (typically at the outlet), and it is used as a conservative measure to ensure a safe $q_{\text {limit }}^{\prime}$ is chosen when doing the calculations for each of the heat rates. This maximum coolant temperature is found by iterating the thermal analysis procedure until a constant value for $T_{\infty, \max }$ is reached (to within 0.1 Kelvin).

$q_{\text {limit }}^{\prime}=\left\{\begin{array}{l}q_{s}^{\prime}, \quad \text { constant heat rate } \\ q_{s}^{\prime} \cos \left(c\left[\left|z-z_{\text {cent }}\right|-x_{0}\right]\right), \text { cosine heat rate profile }\end{array}\right.$

$Q_{\text {limit }}=\min \left(q_{c 1}^{\prime}, q_{c 2}^{\prime}, q_{s}^{\prime}\right),\left\{\begin{array}{c}q_{c 1}^{\prime}=\frac{T_{c 1, l i m i t}-T_{\infty, \max }}{R_{\infty}+R_{c 1}} \\ q_{c 2}^{\prime}=\frac{T_{c 2, \text { limit }}-T_{\infty, \text { max }}}{R_{\infty}+R_{c 1}+R_{g a p}+R_{c 2}} \\ q_{c 2}^{\prime}=\frac{T_{s, \text { limit }}-T_{\infty, \text { max }}}{R_{\infty}+R_{c 1}+R_{g a p}+R_{c 2}+R_{\text {bond }}+R_{r}+R_{S}}\end{array}\right.$

The thermal resistances for each of the components in the system and the coolant are found based on Equation 4a through Equation $4 \mathrm{~g}$, where the basket length $\left(L_{b}\right)$, capsules length $\left(L_{c a p}\right)$, and $L_{s}$ (sample and rodlet clad length) are used alongside the ID of the basket $\left(D_{b i}\right)$, the ODs of Capsules 1 and $2\left(D_{c 1}\right.$ and $D_{c 2}$ respectively), the ODs of the rodlet clad and sample ( $D_{r}$ and $D_{s}$, respectively), and the gas gap 
and thermal bond thicknesses ( $t_{\text {gap }}$ and $t_{\text {bond }}$, respectively). Additionally, the thermal conductivities of Capsule 1, the gas gap, Capsule 2, the thermal bond, the rodlet clad, and the sample are accounted for as well $\left(k_{c 1}, k_{\text {gap }}, k_{c 2}, k_{\text {bond }}, k_{r}\right.$, and $k_{s}$, respectively).

$$
\begin{aligned}
& R_{c 1}=\frac{\ln \left(D_{c 1} /\left[D_{c 2}+2 t_{\text {gap }}\right]\right)}{2 \pi k_{c 1}} \\
& R_{\text {gap }}=\frac{\ln \left(\left[D_{c 2}+2 t_{\text {gap }}\right] / D_{c 2}\right)}{2 \pi k_{\text {gap }}} \\
& R_{c 2}=\frac{\ln \left(D_{c 2} /\left[D_{r}+2 t_{\text {bond }}\right]\right)}{2 \pi k_{c 2}} \\
& R_{\text {bond }}=\frac{\ln \left(\left[D_{r}+2 t_{\text {bond }}\right] / D_{r}\right)}{2 \pi k_{\text {bond }}} \\
& R_{r}=\frac{\ln \left(D_{r} / D_{S}\right)}{2 \pi k_{r}} \\
& R_{S}=\frac{1}{4 \pi k_{s}} \\
& R_{\infty}=\frac{1}{h_{\infty} \pi D_{e q}}
\end{aligned}
$$

Each thermal conductivity is provided by the user as either a constant or as a variable function of temperature for the respective component (sample, Capsule 1, etc.). The user can provide the variable conductivities by providing the function constants for either a power function (Equation 5a) or as a polynomial function up to a $5^{\text {th }}$ order (Equation $5 \mathrm{~b}$ ). We assume that the conductivity (whether constant or variable) is in units of $\frac{W}{m-K}$. If variable conductivities are selected for the sample, the capsules, or the rodlet clad, those conductivities are calculated at each axial location using the maximum temperature of their respective component in the previous axial location. If variable conductivities are selected for the gas gap or the thermal bond, those are calculated at the average temperature of the two surrounding components, where the gas gap is surrounded by Capsule 1 and 2 and the thermal bond is surrounded by Capsule 2 and the rodlet clad.

$$
\begin{aligned}
& k_{\text {power }}(T)=A \cdot B^{C \cdot T} \\
& k_{\text {poly }}(T)=p_{0}+p_{1} T+p_{2} T^{2}+p_{3} T^{3}+p_{4} T^{4}+p_{5} T^{5}
\end{aligned}
$$

$R_{\infty}$ is the only thermal resistance in this model that is due to convection, which is why it requires a special treatment, particularly regarding its convective heat transfer coefficient $\left(h_{\infty}\right)$ and the noted equivalent diameter $\left(D_{e q}\right)$. $D_{e q}$ changes depending on the axial location of the coolant, as shown in Equation 6, while $h_{\infty}$ can be calculated by correlating it to a Nusselt number $(N u)$, the hydraulic diameter $\left(D_{\text {hyd }}\right)$, and the coolant conductivity $\left(k_{\infty}\right)$, as shown in Equation 7. $k_{\infty}$, for this report, is found for water at $2.5 \mathrm{MPa}$, and it changes as a function of the coolant temperature per data from the National Institute of Standards and Technology [19]. 


$$
\begin{aligned}
& D_{e q}=\left\{\begin{array}{cc}
D_{b i}, & \text { above capsule } 1^{\prime} \text { s top surface } \\
D_{b i}-\frac{D_{c 1} L_{c}}{L_{b}}, & \text { below capsule } 1^{\prime} \text { s top surface }
\end{array}\right. \\
& h_{\infty}=\frac{N u k_{\infty}}{D_{h y d}},\left\{\begin{array}{cc}
D_{b i}, & \text { above capsule } 1^{\prime} \text { s top surface } \\
D_{b i}-D_{c 1}, & \text { below capsule } 1^{\prime} \text { s top surface }
\end{array}\right.
\end{aligned}
$$

$N u$ is typically expressed as a function of the Reynolds number $(R e)$ and Prandtl number $(\operatorname{Pr})$ of the coolant, where the $R e$ is a characteristic of the flow, being a multiple of the coolant's density $\left(\rho_{\infty}\right)$, velocity $\left(U_{\infty}\right)$, and $D_{h y d}$ divided by the coolant's dynamic viscosity $\left(\mu_{\infty}\right)$. In this report, five $N u$ correlations are considered, including the Dittus-Boelter correlation in Equation 8 [20], the modified Dittus-Boelter correlation in Equation 9 [21], the Seider-Tate correlation in Equation 10 [22], the Gnielinski correlation in Equation 11 [23], and a custom correlation in Equation 12 developed by combining features in all the aforementioned correlations. Each $N u$ correlation is benchmarked against results from ABAQUS. The reoccurring $f$ in Equation 11 and Equation 12 is the friction factor of the flow in the coolant channel. It should be noted that the user can choose any of the Nusselt number correlations shown in Equation 8-12.

$$
\begin{aligned}
& N u=0.023 \operatorname{Re}^{0.8} \operatorname{Pr}^{0.4} \\
& N u=\left(1+0.912 \operatorname{Re}^{-0.1} \operatorname{Pr}^{0.4}\left[1-2.0043 e^{-D_{\text {hyd }} / D_{c 1}}\right]\right) \cdot 0.023 \operatorname{Re}^{0.8} \operatorname{Pr}^{0.4} \\
& N u=0.027 \operatorname{Re}^{0.8} \operatorname{Pr}^{1 / 3}\left(\frac{\mu_{\infty}}{\mu_{\text {wall }}}\right)^{0.14}, \text { assume } \mu_{\text {wall }}=\mu\left(T_{\infty}+200 \mathrm{~K}\right) \\
& N u=\frac{(f / 8)(\operatorname{Re}-1000) P r}{1+12.7(f / 8)^{0.5}\left(\operatorname{Pr}^{2 / 3}-1\right)} \\
& N u=0.027 \frac{\left(\operatorname{Re}^{0.9}\right)\left(\operatorname{Pr}^{0.7}-1\right)\left(\frac{\mu_{\infty}}{\mu_{\text {wall }}}\right)^{0.14}}{\left|1+\left(20(f / 8)^{0.3} \operatorname{Pr}^{0.25}\right)\right|}
\end{aligned}
$$

The convective nature of the heat transfer between the coolant and Capsule 1 requires the computation of the flow's velocity, as evidenced by the Re presence in every $N u$ correlation needed to calculate $h_{\infty}$. To accommodate this, TOAST asks the user to provide the pressure drop across the basket height $(\Delta P)$, which can then be used to calculate the flow's velocity using a procedure outlined in the INL thermal hydraulics analysis guidebook [24]. The approach utilizes a user-provided $\Delta P$ alongside a calculated friction loss coefficient $\left(K_{f}\right.$, Equation 13) and conservatively assumed contraction and expansion losses $\left(K_{c}=0.5\right.$ and $K_{e}=1$, respectively) to calculate the velocity of the flow by applying the conservation of energy neglecting potential energy (Equation 14).

$$
\begin{aligned}
& K_{f}=\frac{4 f L}{D}, f=\left[-4 \ln \left(\frac{0.27 \epsilon}{D}+\left(\frac{7}{R e}\right)^{0.9}\right)\right]^{-2} \\
& U=\sqrt{\frac{2 \Delta P}{\rho_{\infty}\left(K_{C}+K_{e}+K_{f}\right)}}
\end{aligned}
$$


Due to the approach adopted here, a Reynolds number is necessary in order to calculate the friction loss coefficient $K_{f}$, which presents a conundrum as the Reynolds number is an unknown being solved for. In order to assess that, an analysis was conducted to see how much $K_{f}$ changes with different Reynolds numbers, where the friction factor $(f)$ is calculated using Churchill's equation for a smooth cast-iron pipe $\left(\epsilon=0.26 \mathrm{~mm}\right.$ ) [25]. The results showed that $K_{f}$ reaches a steady state at $R e \geq 10^{4}$ (the expected flow conditions in the core); therefore, $K_{f}$ is always calculated at the conservative $R e$ of $4 \times 10^{6}$ in this study (Figure 6).

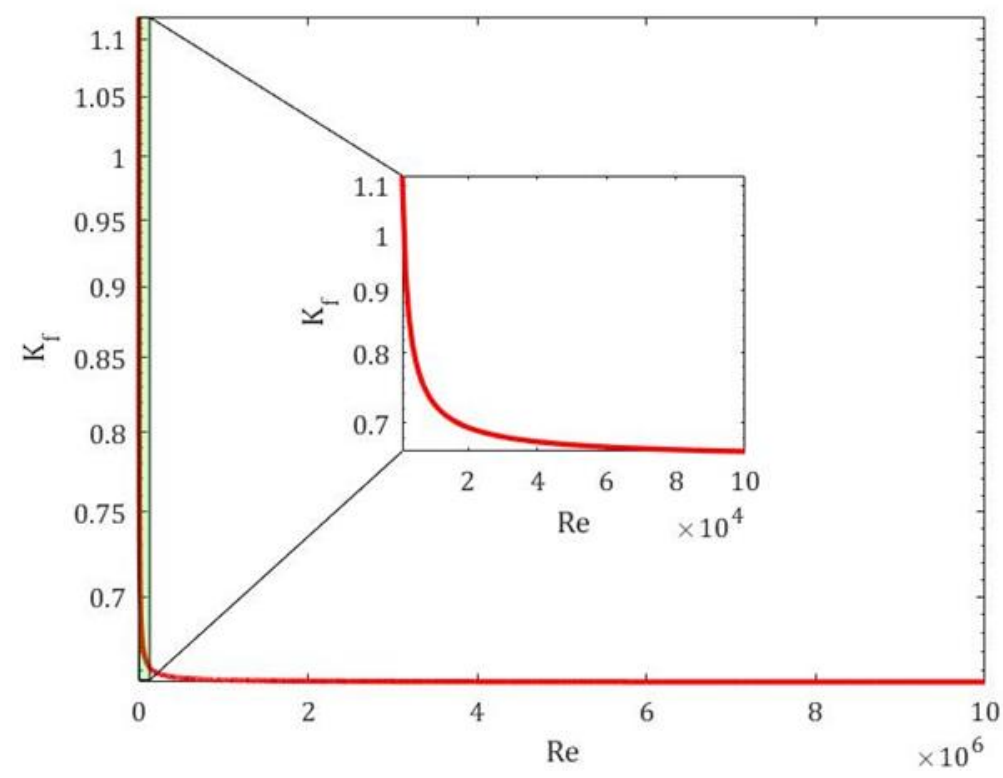

Figure 6. $K_{f}$ evolution as a function of $R e$ using Churchill's equation for the friction factor $f$.

The calculated $U$ would act as the velocity at the axial centerline, from which a mass flow rate is calculated by multiplying it with the density of the coolant and the area of fluid at the centerline. Per the conservation of mass principle, the mass flow rate would remain constant across all axial discretization points, but the changing temperature of the coolant would cause the velocity to change as well to maintain a conserved mass. This allows for the computation of the flow velocity at any axial location using this constant mass flow rate. As such, the Reynolds number at each axial location can be calculated, yielding different $N u$ and $h_{\infty}$ values at each axial location to use for the thermal resistance through the coolant. This completes the thermal resistance circuits that allow for the computation of the axial temperature profiles of each component.

\subsection{Sensitivity Analysis System}

TOAST has a sensitivity and parametric analysis capability, where a user can have one unknown parameter in the thermal analysis model and obtain figures that show the sensitivity of $q^{\prime}{ }_{\text {limit }}$ and $T_{\infty, \max }$ as a function of the changes to that unknown parameter. In some instances, it is also capable of producing plots for the pressure limits of each cylindrical shell (the capsules and the rodlet clad) per the ASME thinwall pressure limits due to circumferential stress on longitudinal joints, as described in the ASME pressure vessel codes [26]. This pressure limit $\left(P_{\text {limit }}\right)$ is calculated as shown in Equation 15, where $S_{y}$ is the yield strength of each component's material, $E$ is the joint efficiency (assume $E=1$ ), $t$ is the thickness of each component, and $D_{i}$ is the inner diameter of the corresponding component. 
$P_{\text {limit }}=\frac{S_{y} E t}{\frac{D_{i}}{2}+0.6 t}$

The ability to track the effects that changes in one unknown parameter has on multiple sensitive values of interest, such as $q_{\text {limit }}^{\prime}, T_{\infty, \max }$, and $P_{\text {limit }}$, simultaneously allows the user to perform thorough optimization studies that account for multiple aspects that are relevant to the design process. As such, it is natural to also consider safety margins for the coolant, such as the departure from nucleate boiling ratio (DNBR) and the flow instability ratio (FIR). DNBR provides a measure of how close the critical heat flux is to the local heat flux of the coolant. To compute it, Equation 16 is used, where $q_{c}^{\prime \prime}$ is the critical heat flux calculated using the correlation laid out by L. Bernath [27], which was used by the Atomic Energy Commission (now superseded by the NRC) [28] and the Arnold Engineering Development Center of the U.S. Air Force [29], and is recommended by the Thermal-Hydraulic Analysis Team (THAT) guidebook [24] for this type of experiment geometry (annular flow channel). The $q_{\max }^{\prime \prime}$ is supposed to be the maximum heat flux that the surface contacting the coolant is emitting, and, as such, it is the maximum heat flux of Capsule 1 (outer capsule). However, as a conservative measure, $q_{\max }^{\prime \prime}$ is set to the maximum heat flux of the sample instead.

$D N B R=\frac{q_{c}^{\prime \prime}}{q_{\max }^{\prime \prime}}=\frac{5.68\left(12915 \frac{D_{\text {hyd }}}{D_{\text {hyd }}+D_{\text {heat }}}+127 \frac{V}{D_{\text {hyd }}^{0.35}}\right)\left(60 \ln (P)-80.8 \frac{P}{P+135}-0.25 V-T_{\infty}\right)}{q_{\max }^{\prime \prime}}$

where $D_{\text {hyd }}[f t]$ is the hydraulic diameter $\left(D_{b i}-D_{c 1}\right), D_{\text {heat }}[f t]$ is the heated diameter $\left(D_{c 1}\right)$, $P[p s i]$ is the coolant pressure, $V[\mathrm{ft} / \mathrm{s}]$ is the coolant velocity (calculated using a total pressure drop), $T_{\infty}\left[{ }^{\circ} \mathrm{C}\right]$ is the coolant temperature (conservatively assumed to be $\left.T_{\infty, \max }\right), q_{c}^{\prime \prime}\left[\mathrm{W} / \mathrm{m}^{2}\right]$ is the critical heat flux, and $q_{\max }^{\prime \prime}\left[\mathrm{W} / \mathrm{m}^{2}\right]$ is the maximum heat flux of the sample (conservatively assumed as $\frac{q_{\text {limit }}^{\prime}}{\pi D_{c 1}}$ ).

It is important to note that, theoretically, if $D N B R>1$ is achieved, $q_{\max }^{\prime \prime}<q_{c}^{\prime \prime}$ and no departure from nucleate boiling is expected. However, it is desirable to use a lower limit that is higher than 1 during the design in order to accommodate any uncertainties or accidents. Those DNBR limits vary from one reactor to another [30], but, for INL's ATR irradiation experiments, that lower limit is 2. As such, it is recommended to maintain $D N B R>2$.

$F I R=\frac{T_{\infty, \text { sat }}-T_{\infty, \text { in }}}{T_{\infty, \text { out }}-T_{\infty, \text { in }}}$

FIR on the other hand provides a measure of how close the coolant's temperature is to its saturation temperature. In other words, how close is the coolant to evaporating at any given pressure. The FIR calculation is performed as shown in Equation 17, where $T_{\infty, s a t}$ is the saturation temperature of the water at the set operating pressure. Per the thermodynamic tables [31], researchers can find the saturation temperature at any desired pressure. $T_{\infty, \text { in }}$ and $T_{\infty, \text { out }}$ are the inlet and outlet temperatures of the coolant, where $T_{\infty, \text { out }}=T_{\infty, \max }$, per the thermal analysis model implemented in TOAST. Much like DNBR, it is desirable to maintain an $F I R>2$ to ensure safe operation with little 2-phase flow instability.

The sensitivity studies that the user is capable of performing in TOAST are in the following list, where each provides the variations in $q_{\text {limit }}^{\prime}\left(Q_{\text {limit }} / L_{s}\right), T_{\infty, \text { max }}$, and $P_{\text {limit }}$ as a function of the changes in the unknown parameter.

1. Sensitivity analysis with respect to $D_{b i}$ 
2. Sensitivity analysis with respect to $D_{c 1}$

3. Sensitivity analysis with respect to $D_{c 2}$

4. Sensitivity analysis with respect to $D_{S}$

5. Sensitivity analysis with respect to $t_{\text {bond }}$
a. Set $T_{\text {s,limit }}$
b. Varied $T_{s, \text { limit }}$

Each sensitivity analysis is expanded upon in the following subsections. Note that some sensitivity analyses have unique features that are not shared with the others. This differentiation is due to the current work's objectives and are not a limitation of TOAST or it potential. Any feature implemented in any of the sensitivity analyses can be implemented in all the other sensitivity analyses as well as any others that may be of interest. More sensitivity analyses can be constructed in the vein of the ones presented here (e.g., consider the analyses listed here as examples for any future sensitivity analysis framework).

\subsubsection{Sensitivity analysis with respect to the basket ID $\left(D_{b i}\right)$}

The user can track changes to $q_{\text {max }}^{\prime}, T_{\infty, \max }$, and $P_{\text {limit }}$ with respect to changes in $D_{b i}$ using the automated procedure presented in Figure 7 . The procedure begins by grabbing the $D_{b o}$ and $D_{c 1}$ userselected values, which serve as the constraints on how large and how small $D_{b i}$ can get. Minimum thicknesses for the annulus and the basket are assumed constant in order to facilitate realistic results. These minimum thicknesses $(0.8 \mathrm{~mm}$ for the basket and $0.1 \mathrm{~mm}$ for the annulus) are currently hardcoded into TOAST, but a future version may provide the user with the ability to change them manually. Another assumption is made regarding the basket material, where the $S_{y}$ of the basket is assumed to be equal to that of stainless-steel 316L (170 MPa). As with the minimum thicknesses, the $S_{y}$ is hardcoded into TOAST but can easily be altered in the source code. A future version of TOAST would provide the user with more control over the sensitivity studies in general. Once the assumptions are made, a minimum $D_{b i}$ is calculated to obtain a minimum fraction with respect to the maximum allowable $D_{b i}\left(D_{b o}-2 t\right)$. This fraction is used to initiate the study at the smallest possible value for $D_{b i}$, continuing until $99 \%$ of the maximum allowable $D_{b i}$ is reached. Once that $99 \%$ mark is reached, two plots are generated containing the linear heat generation rate limit for the sample, the pressure limit of the basket, and the maximum coolant temperature, all as a function of $D_{b i}$. This provides an insight into the effects of a changing $D_{b i}$ on the thermal-hydraulic behavior as well as its effects on the allowable pressure limits on the basket. 


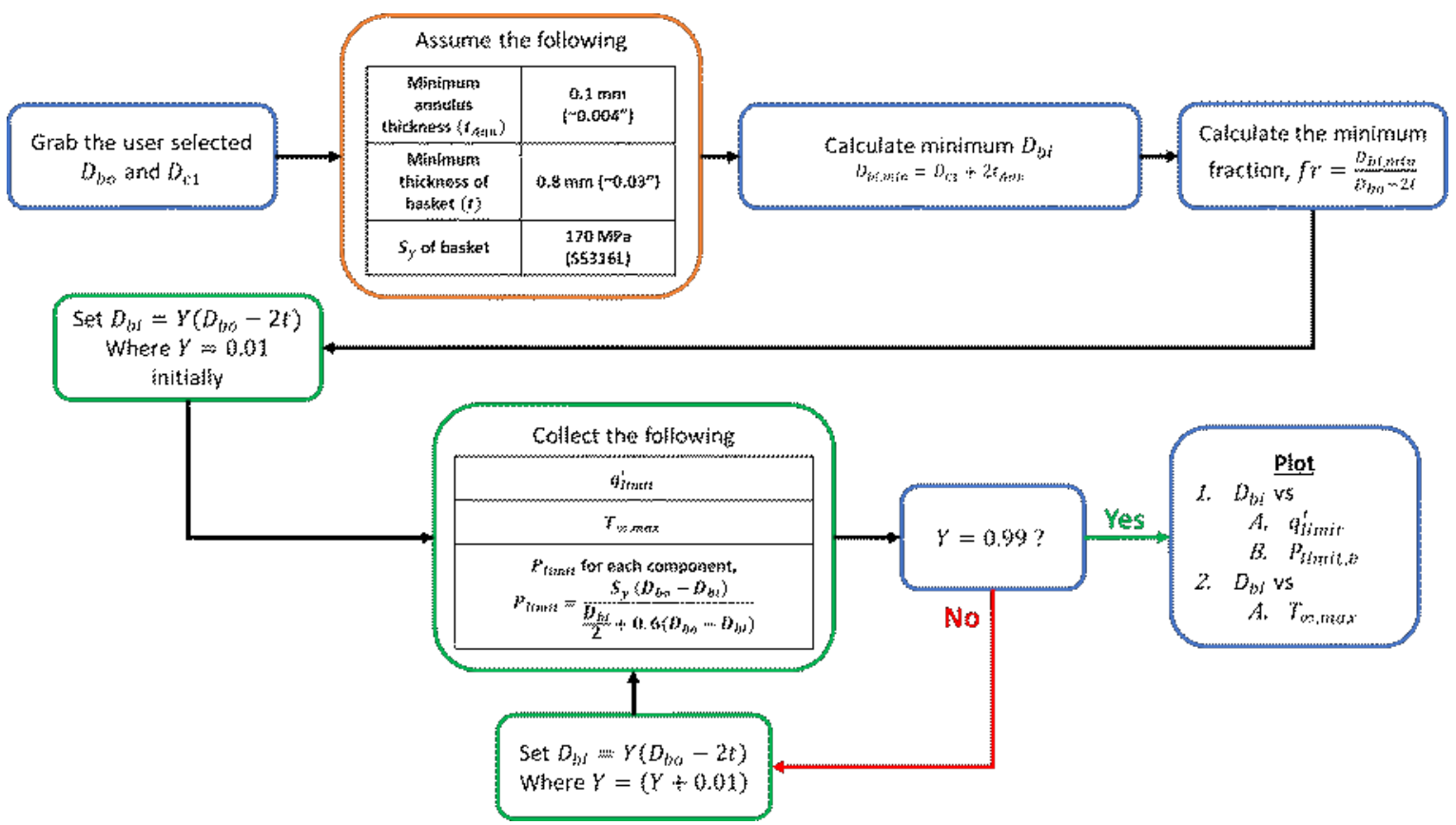

Figure 7. The automated procedure for the sensitivity analysis with respect to $D_{b i}$.

\subsubsection{Sensitivity analysis with respect to the OD of capsule $1\left(D_{c 1}\right)$}

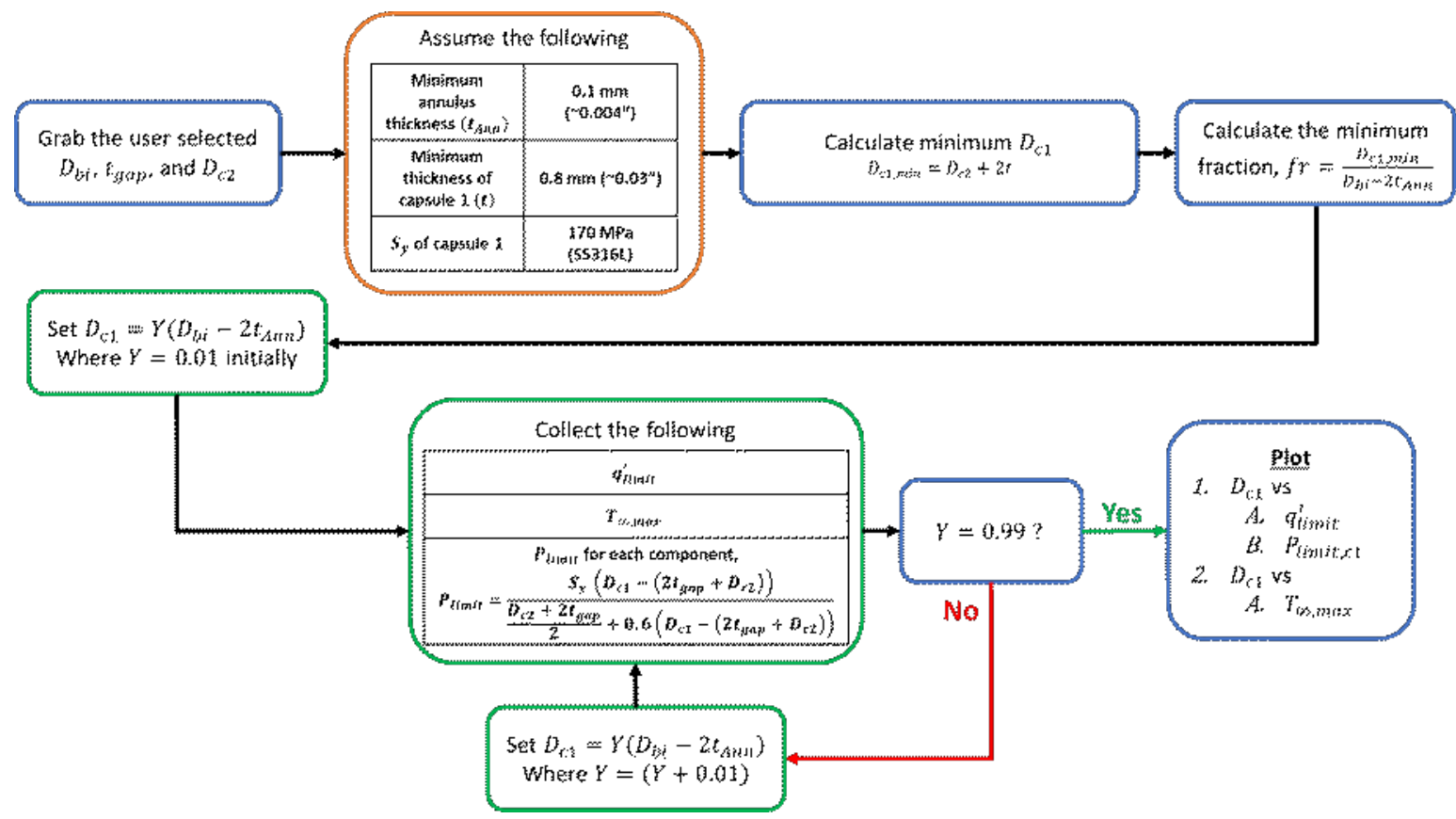

Figure 8 . The automated procedure for the sensitivity analysis with respect to $D_{c 1}$. 
The procedure laid out for $D_{c 1}$ perturbations, shown in Figure 8 , is very similar to that of the $D_{b i}$ procedure with trivial differences in how the minimum $D_{c 1}$ and fraction are calculated and how the results are reported. This provides an insight into the effects of a changing $D_{c 1}$ on the thermal-hydraulic behavior as well as its effects on the allowable pressure limits on the basket.

\subsubsection{Sensitivity analysis with respect to the OD of capsule $2\left(D_{c 2}\right)$}

Like $D_{b i}$ and $D_{c 1}$, the procedure for $D_{c 2}$ includes the same steps with the same trivial differences between them. Figure 9 shows this procedure, which conveniently allows the user to investigate the sensitivity of the sensitive parameters $\left(q_{\text {limit }}^{\prime}, T_{\infty, \max }\right.$, and $\left.P_{\text {limit }}\right)$ with respect to changes in $D_{c 2}$.

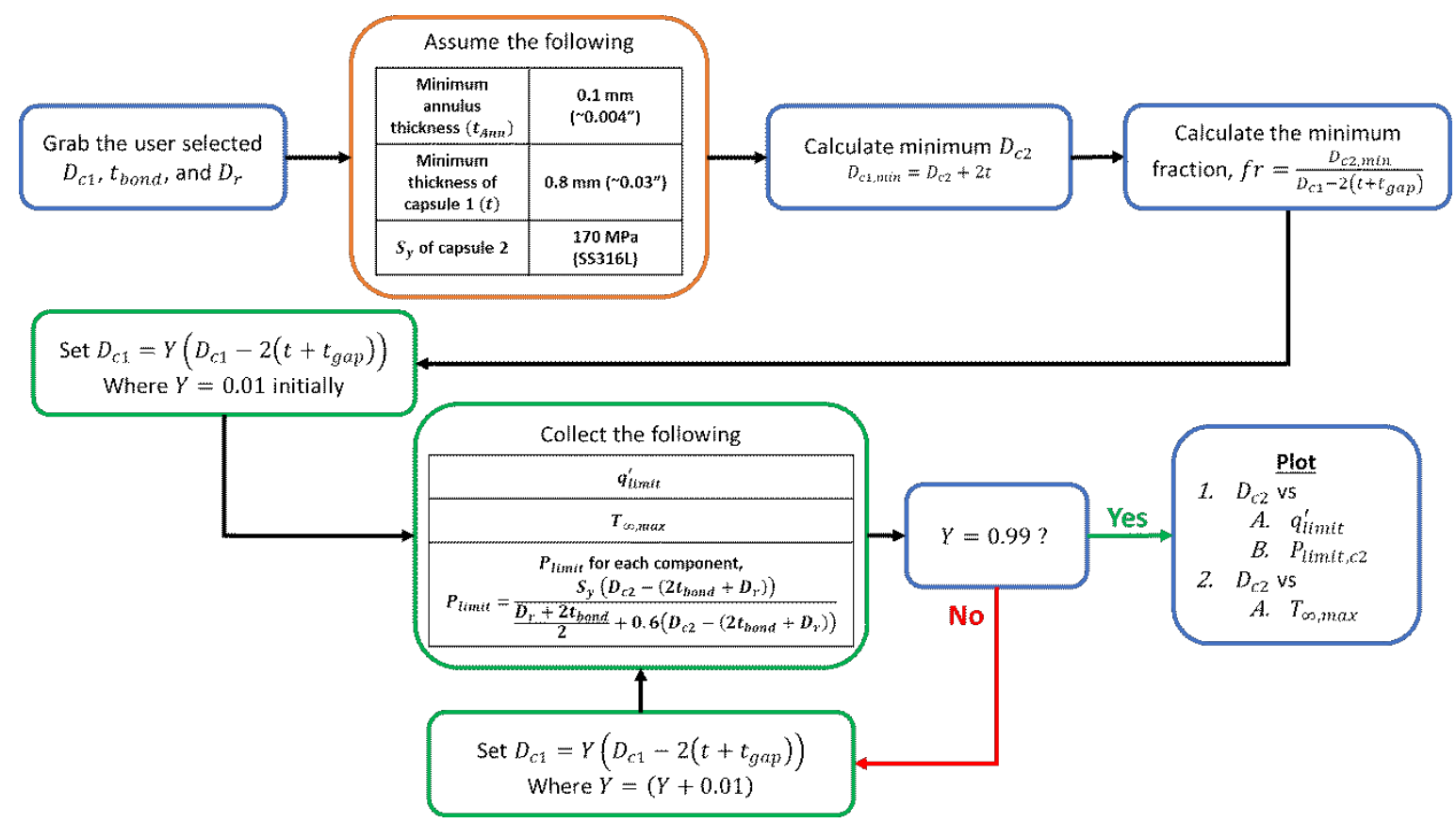

Figure 9. The automated procedure for the sensitivity analysis with respect to $D_{c 2}$.

\subsubsection{Sensitivity analysis with respect to the sample diameter $\left(D_{s}\right)$}

The sample diameter sensitivity analysis procedure, shown in Figure 10, is slightly different than the past three that were introduced. For starters, the system assumes a constant thickness for all the components (capsules and rodlet clad) and calculates a maximum $D_{S}$ and fraction instead of a minimum. In that sense, it starts with the smallest possible $D_{s}$ (assumed to be $1 \%$ of $D_{b i}$ ) and it works its way up to $99 \%$ of the maximum $D_{s}$. Instead of calculating the $P_{\text {limit }}$ for one component, the $D_{s}$ sensitivity analysis calculates it for all the components and puts them on one plot, with the other plot containing the $q_{\text {limit }}^{\prime}$ and $T_{\infty, \max }$ as functions of $D_{s}$. It should be noted that any results reported in this sensitivity analysis reflect their priority to the current work and, if needed, can be easily implemented in each of the sensitivity analyses in the future. 


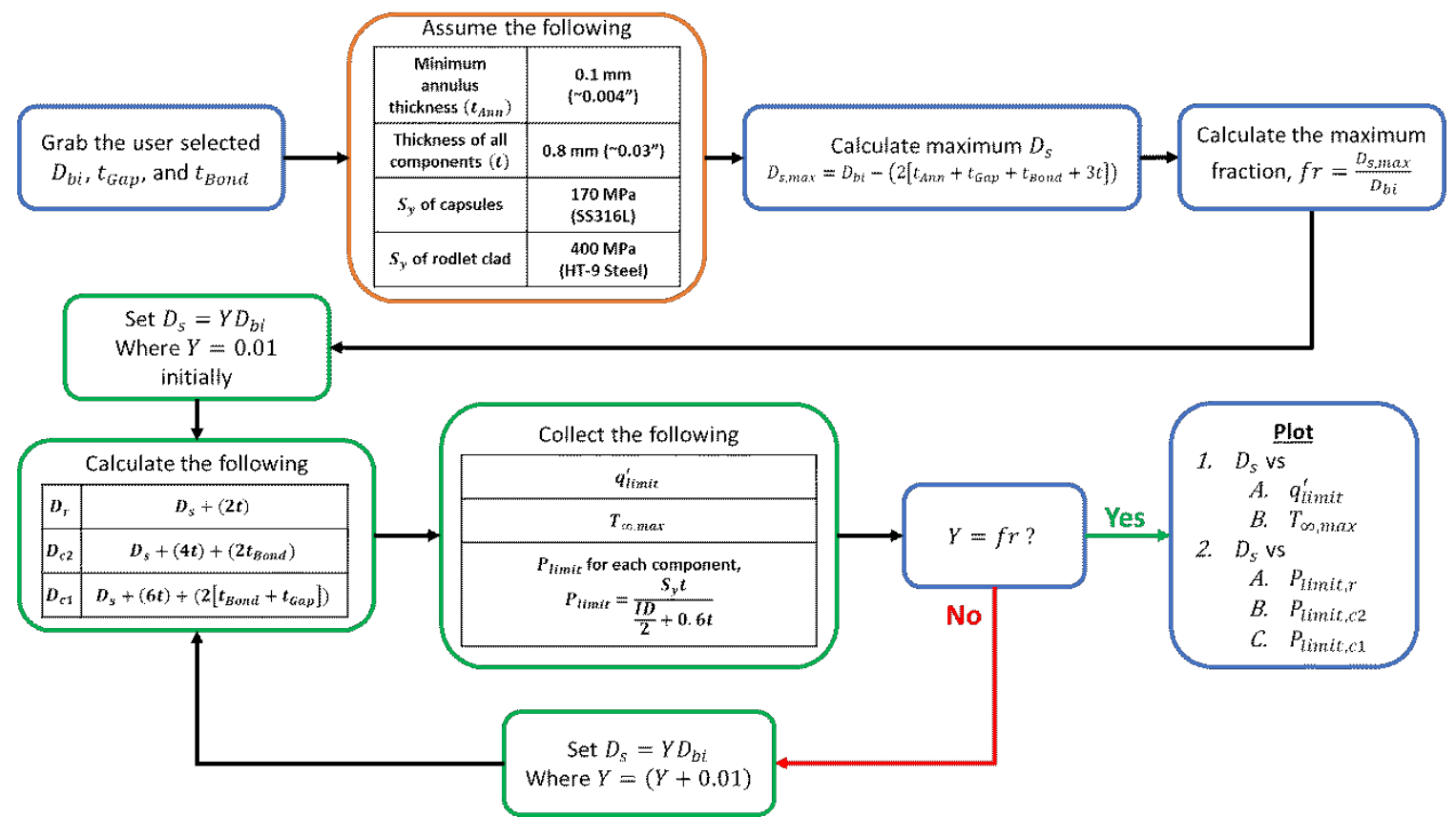

Figure 10. The automated procedure for the sensitivity analysis with respect to $D_{s}$.

\subsubsection{Sensitivity analysis with respect to the bond thickness $\left(t_{\text {bond }}\right)$ with a set sample temperature $\left(T_{s, \text { imit }}\right)$}

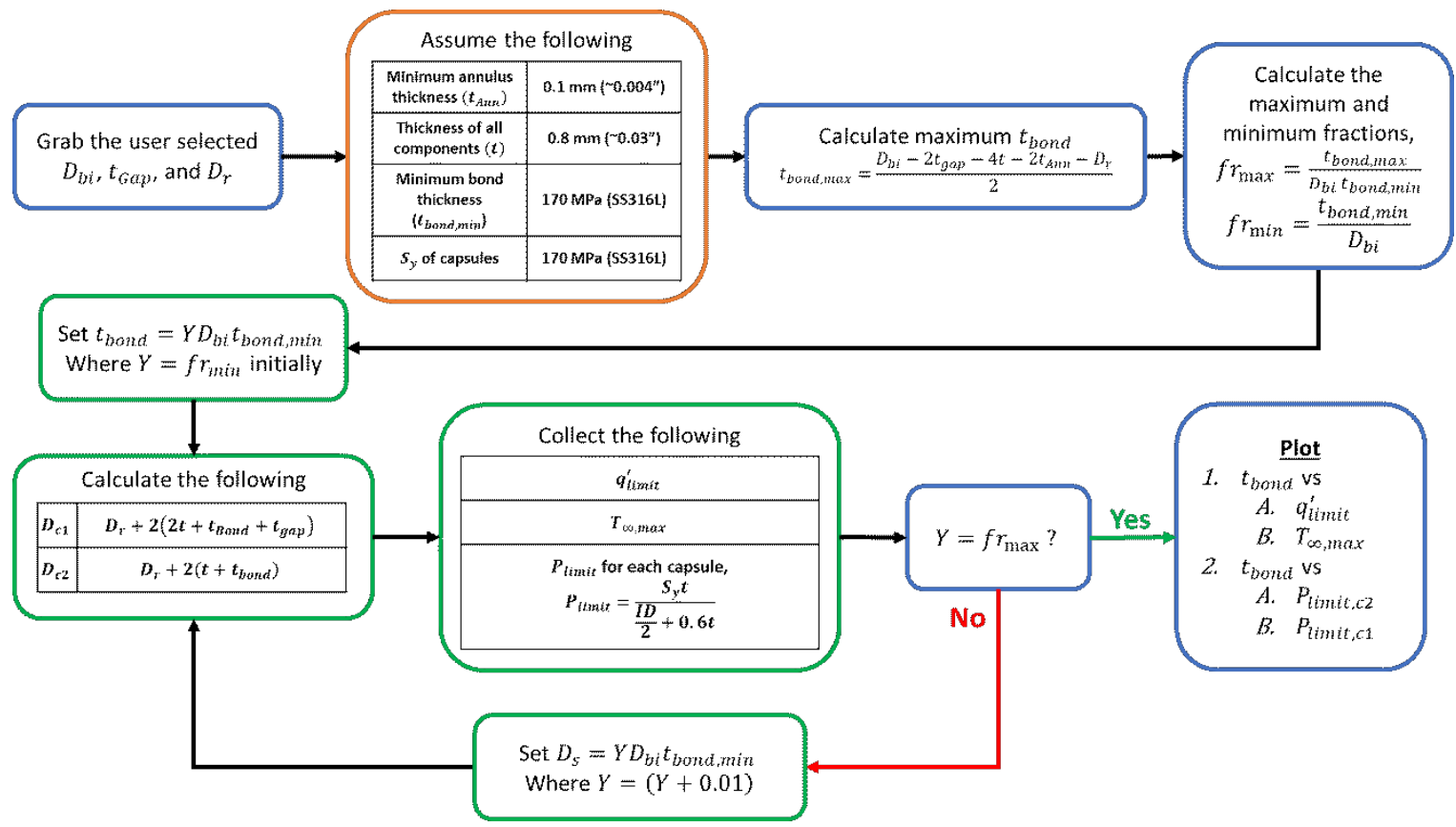

Figure 11. The automated procedure for the sensitivity analysis with respect to $t_{\text {bond }}$. 
The $t_{\text {bond }}$ sensitivity analysis when a set sample temperature limit $\left(T_{s, l i m i t}\right)$ is selected has a very similar procedure to that of $D_{s}$. This is shown in Figure 11, where almost the exact same steps implemented in $D_{s}$ are also implemented here. An exception is how the fraction is used to step through the different $t_{\text {bond }}$ sizes, where both a maximum and an assumed minimum for $t_{b o n d}$ are used to find respective maximum and minimum fractions to bound the $t_{\text {bond }}$ perturbations to. In essence, the $t_{\text {bond }}$ expansion and depression is controlled by the user-provided $D_{b i}, t_{g a p}, D_{r}$, and the thickness of each component (which is assumed to be constant in this work). The $P_{\text {limit }}$ for the affected components (the capsules) are also calculated to show the user how sensitive $P_{\text {limit }}$ is to changes in $t_{\text {bond }}$ (which would affect the IDs of each of the capsules).

\subsubsection{Sensitivity analysis with respect to the bond thickness $\left(t_{\text {bond }}\right)$ with a varied sample temperature limit $\left(T_{s, \text { limit }}\right)$}

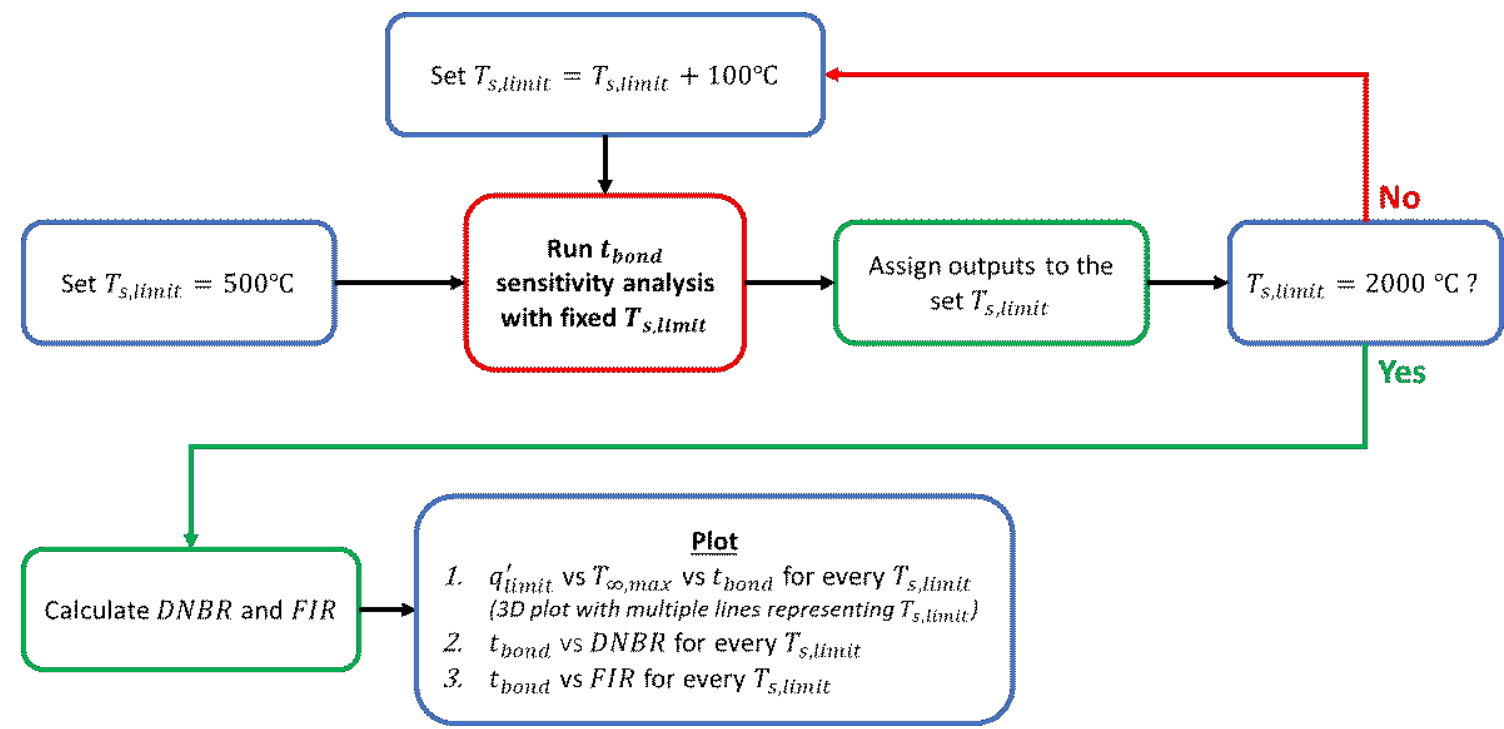

Figure 12. The automated procedure for the sensitivity analysis with respect to $t_{\text {bond }}$ and a varied $T_{s, l i m i t}$.

Due to the demands of this work, an additional feature was enabled for the $t_{\text {bond }}$ sensitivity analysis, enabling the analysis to account for the effects of changing $T_{\text {s,limit }}$ by stepping through an array of values for it as well. In this procedure, shown in Figure 12, TOAST begins by setting a $T_{\text {s,limit }}$ value of $500^{\circ} \mathrm{C}$ and running the $t_{\text {bond }}$ sensitivity analysis with a constant $T_{s, l i m i t}$, as shown in Figure 11 . The results are then stored in a directory specific to that selected $T_{s, l i m i t}$ in order to assist with the postprocessing that occurs later. Once the outputs are stored appropriately, the code increases the $T_{\text {s,limit }}$ by an increment of $100^{\circ} \mathrm{C}$ and repeats the process until a $T_{s, l i m i t}$ of $2000^{\circ} \mathrm{C}$ is reached. The postprocessing begins after this by calculating the $D N B R$ and $F I R$ using the stored information for each temperature at all potential $t_{\text {bond }}$ sizes. After the successful calculation of $D N B R$ and $F I R$, three plots are generated, the first of which is a 3D plot with $T_{\infty, \max }$ on the x-axis, $q_{\text {limit }}^{\prime}$ on the y-axis, and $t_{\text {bond }}$ on the z-axis with multiple curves plotted (each of which corresponds to a different $T_{\text {s,limit }}$ ). The other two plots are for the DNBR and FIR respectively as a function of $t_{\text {bond }}$ for multiple $T_{s, l i m i t}$ values (multiple curves). 


\subsection{Graphical User Interface}

TOAST is presented to the user via a simple and highly accessible graphical user interface (GUI) that provides flexibility when determining the specifics of the studies. The GUI consists of two tabs that are shown in Figure 13 and Figure 14, respectively, and it is created in MATLAB along with the rest of the TOAST framework. Starting with the Main Settings tab in Figure 13, the GUI consists of 11 user inputs for the geometry definition (any or none of which can be set to zero); three temperature limit inputs (for both capsules and the sample); five thermal conductivity inputs for all the components including the basket (although the basket conductivity is currently unused in this version); three flow behavior inputs (pressure, pressure drop, and inlet temperature); and one drop down menu to select the number of axial discretization points. It also includes four labels, one to provide the status of the thermal model (top left), one to provide the status of the sensitivity study that is being run (bottom left), and two labels to express the $T_{\infty, \max }$ and $q_{\text {limit }}^{\prime}$ (bottom right). There are two plots in the GUI (both in the Main Settings tab): the first visualizes the user-defined geometry (top right), and the second plot shows the half-axial temperature distributions (top of coolant channel to the axial centerline) for each of the components (except for the basket, which is assumed to be negligible) and the coolant temperature. Additionally, a coolant temperature full-axial distribution (spanning the entire height of the coolant channel) is plotted on an external figure.

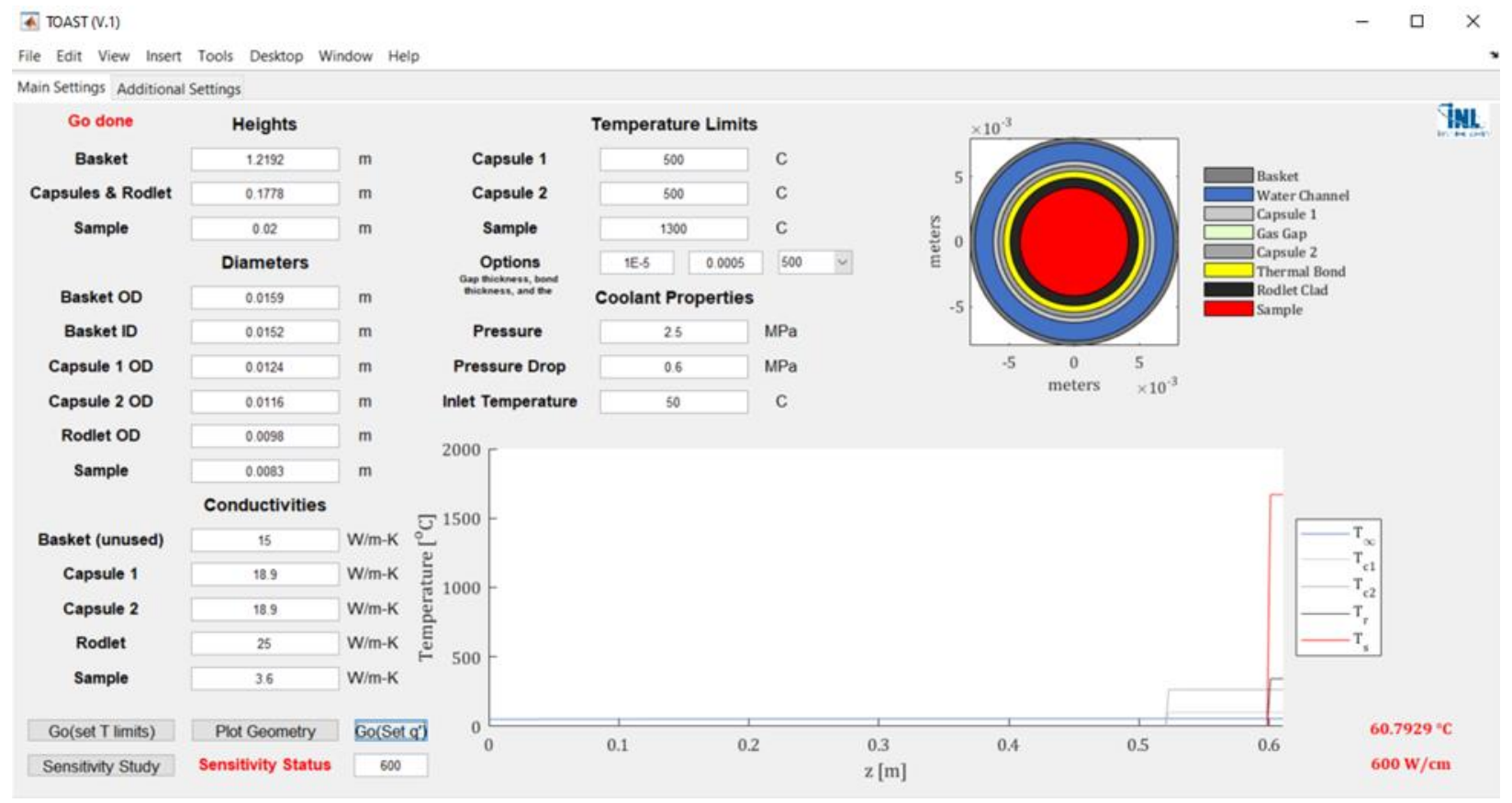

Figure 13. The Main Settings tab of the GUI for TOAST.

The user is provided with four buttons on the bottom-left portion of the Main Settings tab, each initiating a different function when pressed. The Go (set T limits) button allows the user to run the thermal analysis model with their selected geometry, conductivities, flow conditions, and discretization points with the set temperature limits for the capsules and the sample such that Equation $3 \mathrm{~b}$ is used to determine the $q_{\text {limit }}^{\prime}$. The Go (set $q^{\prime}$ ) button on the other hand allows the user to run the thermal analysis model with a predefined $q_{\text {limit }}^{\prime}(\mathrm{W} / \mathrm{cm})$ in the white blank underneath the button. The Plot Geometry button does exactly what the name suggests in that it only plots the geometry in the top right of the Main Settings tab for visualization purposes only.

Finally, the Sensitivity Study button allows the user to run the sensitivity analyses described in section 3.2 of this report. This is done by having the user place a question mark symbol (?) in the white text 
boxes corresponding to the sensitivity analysis of interest, which are currently limited to the basket ID, capsule 1 and capsule 2 OD values, Sample diameter, Bond thickness, or the combination of Bond thickness and Sample temperature limits. When the user presses the Sensitivity Study button after placing the question mark symbol in the appropriate place(s), TOAST automatically proceeds with the sensitivity analysis of choice per the procedures laid out in section 3.2.

As mentioned earlier in Section Thermal-Hydraulic Analysis Model, the user has ability to prescribe the conductivity of any of the components as functions of their temperatures, and this is accommodated in the Additional Settings tab of the GUI, which is shown in Figure 14. The user can prescribe the constants for either a power function or a polynomial function of up to the $5^{\text {th }}$ order (per Equation $5 \mathrm{a}$ and $5 \mathrm{~b}$, respectively). On this Additional Settings tab, the user can also choose the heat generation behavior by picking whether they desire a constant $q_{s}^{\prime}$ or a cosine-shaped $q_{s}^{\prime}$. Finally, at the very bottom of the screen, the user can choose from any of the five available $N u$ correlations, where each correlation is listed in the order corresponding to Equation 8-12 such that the Dittus-Boelter (1930) correlation corresponds to Equation 8 and the AWeiss (2020) correlation corresponds to the custom correlation that is Equation 12.

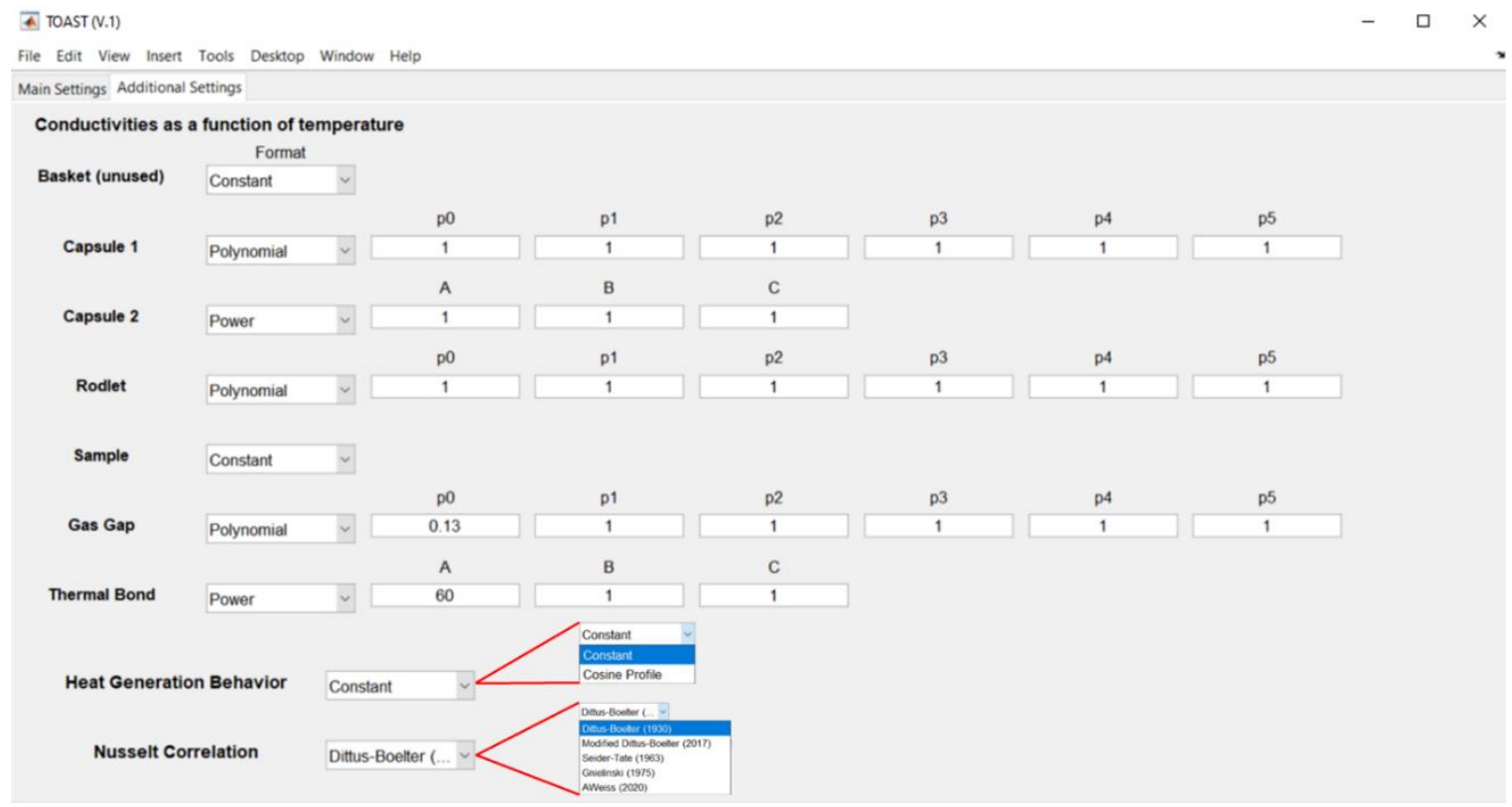

Figure 14.The Additional Settings tab of the GUI for TOAST.

It should be noted that TOAST automatically outputs the coolant axial temperature distribution and a .csv file with all the $q_{\text {limit }}^{\prime}$ and temperature distributions shown in the bottom plot of the GUI. The interests of this current work allowed the development of TOAST to be carried out in MATLAB, which allows for a variety of built-in accessibility functions, allowing any user to manually save the plots or even customize the GUI with ease. A future version of TOAST can be easily (relatively) redeveloped in more opensource or readily accessible environments, such as Python, by porting the existing MATLAB functions into Python scripts.

This concludes the description of TOAST and its capabilities, which are used in the following sections describing the benchmark (providing an uncertainty of the code) and the optimization results for the NMDQi experiments. 


\section{BENCHMARK}

\subsection{Benchmark Setup}

In an effort to verify the results from TOAST for the NMDQi experiments, with which most of the future parametric and sensitivity studies shall be conducted, a set of benchmark problems were solved using TOAST and the commercial software ABAQUS. The results from TOAST are compared directly to ABAQUS to provide a better understanding of TOAST's accuracy with respect to the other two software packages. The benchmarks are inspired by previous thermal-hydraulic analyses of accident tolerant fuels (ATFs) irradiation [6] and the Advanced Fuels Campaign Fission Accelerated Steady-State Testing (AFC-FAST) thermal-hydraulic analyses [32].

The benchmarks are set up as follows, where a user-supplied constant $q_{\text {limit }}^{\prime}$ of 200, 225, 250, 275, and $300 \frac{\mathrm{W}}{\mathrm{cm}}$ are provided for each of the setups.

1. U-10Zr Fuel - Constant conductivities $\left(\frac{W}{m-K}\right)$
a. $k_{\text {sample }}=22$
b. $k_{r}=k_{c 1}=k_{c 2}=13$
c. $k_{\text {gap }}=0.13$
d. $k_{\text {bond }}=60$

2. U-10Zr Fuel - Variable conductivities $\left(\frac{W}{m-K}\right)$
a. $\quad k_{\text {sample }}=11.71188+(0.01334 T)+\left(9.38 \times 10^{-6} T^{2}\right)$
b. $k_{r}=29.65-(0.06668 T)+\left(2.184 \times 10^{-4} T^{2}\right)-\left(2.527 \times 10^{-7} T^{3}\right)+\left(9.621 \times 10^{-11} T^{5}\right)$
c. $k_{c 1}=k_{c 2}=8.38266+(0.01750316 T)-\left(3.146906 \times 10^{-6} T^{2}\right)$
d. $\quad k_{\text {gap }}=0.06187+\left(2.89714 \times 10^{-4} \mathrm{~T}\right)$
e. $\quad k_{\text {bond }}=111.5731-(0.07026424 T)+\left(2.373302 \times 10^{-5} T^{2}\right)-\left(8.567 \times 10^{-9} T^{3}\right)+$ $\left(2.025858 \times 10^{-12} T^{4}\right)-\left(2.163437 \times 10^{-16} T^{5}\right)$

The thermal conductivity function for the sample is based on a $\mathrm{U}-10 \mathrm{Zr}$ sample with $0 \%$ porosity, based on models suggested by Billone et al. [33]. The rodlet clad assumes an HT-9 steel material whose thermal conductivity function is suggested by Argonne National Laboratory [34]. Both capsules are assumed to be made of stainless-steel $316 \mathrm{~L}$ with the conductivity function based on data from the works of Umezawa et al. [35] and Lucks et al. [36]. For the gas gap, we assume 100\% He gas, with a thermal conductivity function from the works of Siefken et al. [37]. The thermal bond was liquid sodium, with a thermal conductivity function based on the works of Ho et al. [38]. It should be noted that all those thermal conductivity models are the ones used by the BISON [39] code package within the Multiphysics Object-Oriented Simulation Environment (MOOSE) Multiphysics framework [40]. 
For all the benchmarks, the following characteristics apply.

\begin{tabular}{c|c}
\hline Characteristic & Value \\
\hline Basket ID $\left(\boldsymbol{D}_{\boldsymbol{b i}}\right)$ & $14.0208 \mathrm{~mm}(0.552 \mathrm{in})$ \\
Capsule 1 OD $\left(\boldsymbol{D}_{\boldsymbol{c} \mathbf{1}}\right)$ & $10.9728 \mathrm{~mm}(0.432 \mathrm{in})$ \\
Capsule 2 OD $\left(\boldsymbol{D}_{\boldsymbol{c} \mathbf{2}}\right)$ & $9.4488 \mathrm{~mm}(0.372 \mathrm{in})$ \\
Rodlet clad OD $\left(\boldsymbol{D}_{\boldsymbol{r}}\right)$ & $2.921 \mathrm{~mm}(0.115 \mathrm{in})$ \\
Sample OD $\left(\boldsymbol{D}_{\boldsymbol{s}}\right)$ & $2.4638 \mathrm{~mm}(0.097 \mathrm{in})$ \\
\hline Basket length $\left(\boldsymbol{L}_{\text {Basket }}\right)$ & $2.1336 \mathrm{~m}(84 \mathrm{in})$ \\
$\boldsymbol{L}_{\text {capsules }}=\boldsymbol{L}_{\boldsymbol{c l a d}}=\boldsymbol{L}_{\text {sample }}$ & $15.6464 \mathrm{~cm}(6.16 \mathrm{in})$ \\
\hline Gap thickness $\left(\boldsymbol{t}_{\boldsymbol{g a p}}\right)$ & $0.0508 \mathrm{~mm}(0.002 \mathrm{in})$ \\
Thermal bond thickness $\left(\boldsymbol{t}_{\text {bond }}\right)$ & $2.8829 \mathrm{~mm}(0.113 \mathrm{in})$ \\
\hline$\Delta \boldsymbol{P}_{\text {across basket }}\left[\boldsymbol{U}_{\text {coolant }}\right]$ & $0.08193 \mathrm{MPa} \quad[8.53 \mathrm{~m} / \mathrm{s}]$ \\
Coolant pressure $\left(\boldsymbol{P}_{\text {coolant }}\right)$ & $2.5 \mathrm{MPa}$ \\
$\boldsymbol{T}_{\text {coolant,inlet }}=\boldsymbol{T}_{\infty, \text { in }}$ & $50{ }^{\circ} \mathrm{C}$ \\
\hline
\end{tabular}

The ABAQUS simulations consisted of a 2D axisymmetric model with approximately 155,000 quadrilateral heat transfer elements in the mesh, with grading in and out of the gas gap. The coolant in the simulation is modeled as a constant sink temperature with a heat transfer coefficient that is a function of the film temperature. The ABAQUS simulations are run on a local windows machine with a quad-core i7$8665 \mathrm{U}$ processor and $16 \mathrm{~GB}$ of RAM. Each ABAQUS simulation took hours to set up and $<5$ minutes to run on the local machine.

Results from the TOAST simulation will consider 250 axially discretized points from the top of the irradiation basket to the axial centerline. Axial and radial symmetry about the centerline is assumed. A constant heat generation profile is assumed for all benchmark cases, wherein $q_{\text {limit }}^{\prime}$ does not vary with the height of the sample. Different $N u$ correlations are benchmarked here as well, all of which were listed earlier with Equations 8-12 in Section Thermal-Hydraulic Analysis Model. The purpose of using different $N u$ correlations is to highlight the overall sensitivity of the thermal behavior to $N u$ variance and, therefore, the $h_{\infty}$ uncertainty. It should be noted that each computation in TOAST took anywhere between a few seconds to nearly a couple minutes to set up (versus the hours it takes for ABAQUS), and it ran within seconds (5-30 seconds depending on the user-selected number of axial discretization points) on a local machine with a 2-core i5-7200U processor and 12 GB of RAM.

\subsection{Benchmark Results}

The ABQUS results are compared to results from TOAST with the five different $N u$ correlations shown in Equations 8-12, respectively (i.e., TOAST $1=$ Equation 8, TOAST 2 = Equation 9, etc.). The benchmarked temperatures are for Capsule 1's inner temperature $\left(T_{c 1}\right)$, Capsule 2's outer temperature $\left(T_{c 2, \text { out }}\right)$, the rodlet clad inner temperature $\left(T_{r}\right)$, and the sample centerline temperature $\left(T_{s}\right)$. Table 1 shows the results for the benchmarks with constant thermal conductivities, and Table 2 shows the corresponding percent difference $\left(\epsilon_{c 1}, \epsilon_{c 2, o u t}, \epsilon_{r}, \epsilon_{s}\right)$ between the ABAQUS temperature results and the results from each TOAST computation. Similarly, Table 3 and Table 4 correspond to the benchmark cases with the prescribed variable thermal conductivities. 
Table 1. Constant conductivity benchmarks.

\begin{tabular}{|c|c|c|c|c|c|c|}
\hline Case & $\boldsymbol{q}_{\text {limit }}^{\prime}(\boldsymbol{W} / \mathrm{cm})$ & Computation & $T_{c 1}\left({ }^{\circ} \mathrm{C}\right)$ & $\boldsymbol{T}_{c 2, \text { out }}\left({ }^{\circ} \mathrm{C}\right)$ & $T_{r}\left({ }^{\circ} \mathrm{C}\right)$ & $T_{s}\left({ }^{\circ} \mathrm{C}\right)$ \\
\hline \multirow{6}{*}{1} & \multirow{6}{*}{200} & ABAQUS & 111.34 & 373.13 & 493.20 & 565.54 \\
\hline & & TOAST 1 & 110.29 & 372.17 & 492.25 & 564.60 \\
\hline & & TOAST 2 & 113.65 & 375.52 & 495.61 & 567.95 \\
\hline & & TOAST 3 & 105.83 & 367.71 & 487.80 & 560.14 \\
\hline & & TOAST 4 & 109.33 & 371.21 & 491.29 & 563.64 \\
\hline & & TOAST 5 & 108.88 & 370.76 & 490.85 & 563.19 \\
\hline \multirow{6}{*}{2} & \multirow{6}{*}{225} & ABAQUS & 117.03 & 411.54 & 546.62 & 628.01 \\
\hline & & TOAST 1 & 117.72 & 412.33 & 547.43 & 628.82 \\
\hline & & TOAST 2 & 121.40 & 416.01 & 551.11 & 632.49 \\
\hline & & TOAST 3 & 112.77 & 407.39 & 542.49 & 623.87 \\
\hline & & TOAST 4 & 116.66 & 411.28 & 546.37 & 627.76 \\
\hline & & TOAST 5 & 116.40 & 411.01 & 546.11 & 627.49 \\
\hline \multirow{6}{*}{3} & \multirow{6}{*}{250} & ABAQUS & 122.71 & 449.94 & 600.03 & 690.46 \\
\hline & & TOAST 1 & 125.13 & 452.48 & 602.59 & 693.02 \\
\hline & & TOAST 2 & 129.11 & 456.46 & 606.57 & 697.00 \\
\hline & & TOAST 3 & 119.71 & 447.05 & 597.16 & 687.59 \\
\hline & & TOAST 4 & 123.98 & 451.33 & 601.44 & 691.87 \\
\hline & & TOAST 5 & 123.95 & 451.30 & 601.40 & 691.83 \\
\hline \multirow{6}{*}{4} & \multirow{6}{*}{275} & ABAQUS & 128.37 & 488.32 & 653.42 & 752.89 \\
\hline & & TOAST 1 & 132.55 & 492.64 & 657.76 & 757.23 \\
\hline & & TOAST 2 & 136.83 & 496.91 & 662.03 & 761.50 \\
\hline & & TOAST 3 & 126.66 & 486.74 & 651.86 & 751.33 \\
\hline & & TOAST 4 & 131.32 & 491.41 & 656.53 & 756.00 \\
\hline & & TOAST 5 & 131.57 & 491.66 & 656.78 & 756.25 \\
\hline \multirow{6}{*}{5} & \multirow{6}{*}{300} & ABAQUS & 134.01 & 526.69 & 706.79 & 815.31 \\
\hline & & TOAST 1 & 139.78 & 532.60 & 712.73 & 821.24 \\
\hline & & TOAST 2 & 144.22 & 537.03 & 717.17 & 825.68 \\
\hline & & TOAST 3 & 133.50 & 526.32 & 706.45 & 814.97 \\
\hline & & TOAST 4 & 138.50 & 531.32 & 711.45 & 819.97 \\
\hline & & TOAST 5 & 139.41 & 532.23 & 712.36 & 820.87 \\
\hline
\end{tabular}

The constant thermal conductivity cases show that Dittus-Boelter (Equation 8) works particularly well at lower $q_{\text {limit }}^{\prime}$ values and tend to lose accuracy as the $q_{\text {limit }}^{\prime}$ increases. Modified Dittus-Boelter (Equation 9) works similarly to Dittus-Boelter with respect to increasing the $q_{\text {limit }}^{\prime}$; however, it leads to less accurate results, particularly at $T_{c 1}$, which is where the coolant effect should be most noticeable and, hence, where the $N u$ (and $h_{\infty}$ by extension) matters the most. This indicates that the modified DittusBoelter correlation may not be desirable for this kind of thermal problem on account of its inaccurate $h_{\infty}$ prediction and its inaccurate depiction of the convection heat transfer between the coolant and Capsule 1 (the outer capsule). 
Seider-Tate (Equation 10) tends to work inversely to the Dittus-Boelter correlations with respect to an increasing $q_{\text {limit }}^{\prime}$. As the sample gets hotter, lower $\epsilon$ values are observed across all temperatures. It is noteworthy however that, at a lower $q_{\text {limit }}^{\prime}$, Seider-Tate tends to be noticeably more inaccurate than all other $N u$ correlations. This indicates that Seider-Tate is likely a desirable $N u$ correlation if high $q_{\text {limit }}^{\prime}$ values are expected, as it performs best compared to its alternatives, but it is highly undesirable at lower $q_{\text {limit }}^{\prime}$ values.

Table 2. Percent difference between ABAQUS and TOAST for the constant conductivity benchmarks.

\begin{tabular}{|c|c|c|c|c|c|c|}
\hline Case & $\boldsymbol{q}_{\text {limit }}^{\prime}(\boldsymbol{W} / \mathrm{cm})$ & Computation & $\epsilon_{c 1}$ & $\epsilon_{c 2, o u t}$ & $\epsilon_{r}$ & $\epsilon_{s}$ \\
\hline \multirow{5}{*}{1} & \multirow{5}{*}{200} & TOAST 1 & $-0.94 \%$ & $-0.26 \%$ & $-0.19 \%$ & $-0.17 \%$ \\
\hline & & TOAST 2 & $2.07 \%$ & $0.64 \%$ & $0.49 \%$ & $0.43 \%$ \\
\hline & & TOAST 3 & $-4.95 \%$ & $-1.45 \%$ & $-1.10 \%$ & $-0.95 \%$ \\
\hline & & TOAST 4 & $-1.81 \%$ & $-0.52 \%$ & $-0.39 \%$ & $-0.34 \%$ \\
\hline & & TOAST 5 & $-2.21 \%$ & $-0.63 \%$ & $-0.48 \%$ & $-0.42 \%$ \\
\hline \multirow{5}{*}{2} & \multirow{5}{*}{225} & TOAST 1 & $0.59 \%$ & $0.19 \%$ & $0.15 \%$ & $0.13 \%$ \\
\hline & & TOAST 2 & $3.73 \%$ & $1.09 \%$ & $0.82 \%$ & $0.71 \%$ \\
\hline & & TOAST 3 & $-3.64 \%$ & $-1.01 \%$ & $-0.76 \%$ & $-0.66 \%$ \\
\hline & & TOAST 4 & $-0.31 \%$ & $-0.06 \%$ & $-0.05 \%$ & $-0.04 \%$ \\
\hline & & TOAST 5 & $-0.54 \%$ & $-0.13 \%$ & $-0.09 \%$ & $-0.08 \%$ \\
\hline \multirow{5}{*}{3} & \multirow{5}{*}{250} & TOAST 1 & $1.97 \%$ & $0.56 \%$ & $0.43 \%$ & $0.37 \%$ \\
\hline & & TOAST 2 & $5.22 \%$ & $1.45 \%$ & $1.09 \%$ & $0.95 \%$ \\
\hline & & TOAST 3 & $-2.45 \%$ & $-0.64 \%$ & $-0.48 \%$ & $-0.42 \%$ \\
\hline & & TOAST 4 & $1.04 \%$ & $0.31 \%$ & $0.24 \%$ & $0.20 \%$ \\
\hline & & TOAST 5 & $1.01 \%$ & $0.30 \%$ & $0.23 \%$ & $0.20 \%$ \\
\hline \multirow{5}{*}{4} & \multirow{5}{*}{275} & TOAST 1 & $3.26 \%$ & $0.88 \%$ & $0.66 \%$ & $0.58 \%$ \\
\hline & & TOAST 2 & $6.59 \%$ & $1.76 \%$ & $1.32 \%$ & $1.14 \%$ \\
\hline & & TOAST 3 & $-1.33 \%$ & $-0.32 \%$ & $-0.24 \%$ & $-0.21 \%$ \\
\hline & & TOAST 4 & $2.30 \%$ & $0.63 \%$ & $0.48 \%$ & $0.41 \%$ \\
\hline & & TOAST 5 & $2.50 \%$ & $0.68 \%$ & $0.51 \%$ & $0.45 \%$ \\
\hline \multirow{5}{*}{5} & \multirow{5}{*}{300} & TOAST 1 & $4.30 \%$ & $1.12 \%$ & $0.84 \%$ & $0.73 \%$ \\
\hline & & TOAST 2 & $7.62 \%$ & $1.96 \%$ & $1.47 \%$ & $1.27 \%$ \\
\hline & & TOAST 3 & $-0.38 \%$ & $-0.07 \%$ & $-0.05 \%$ & $-0.04 \%$ \\
\hline & & TOAST 4 & $3.35 \%$ & $0.88 \%$ & $0.66 \%$ & $0.57 \%$ \\
\hline & & TOAST 5 & $4.03 \%$ & $1.05 \%$ & $0.79 \%$ & $0.68 \%$ \\
\hline
\end{tabular}

Gnielinski (Equation 11), alongside the custom correlation (Equation 12) seem to be the most balanced options across all $q_{\text {limit }}^{\prime}$ values, with Gnielinski's correlation being slightly more accurate than the custom correlation. It is notable however that, just like the Dittus-Boelter and modified Dittus-Boelter correlations, the $\epsilon$ for the Gnielinski and custom correlation seems to increase with an increasing $q_{\text {limit }}^{\prime}$ but with a lower rate. This indicates that the Gnielinski and custom correlations are likely most desirable in intermediate $q_{\text {limit }}^{\prime}$ values, where higher $\epsilon$ values may be found with any other correlation. 
It should be noted however that, across most of the current benchmarks, $\epsilon$ tends to not exceed $8 \%$, which is very good considering the purpose of TOAST is to provide a quick, computationally inexpensive, and fairly accurate framework for conducting thermal analyses, particularly for optimization and programmatic studies. Moreover, the sample centerline temperature, which will almost always be the maximum temperature in the geometry, is predicted with an $\epsilon<1 \%$ across all $N u$ correlations, which is astonishing considering the simplified nature of TOAST. This proves TOAST's ability to perform preand mid-irradiation thermal safety, programmatic, and optimization analyses, particularly for typical irradiation experiments in the ATR.

Table 3. Variable conductivity benchmarks.

\begin{tabular}{|c|c|c|c|c|c|c|}
\hline Case & $\boldsymbol{q}_{\text {limit }}^{\prime}(\boldsymbol{W} / \mathrm{cm})$ & Computation & $T_{c 1}\left({ }^{\circ} \mathrm{C}\right)$ & $T_{c 2, \text { out }}\left({ }^{\circ} \mathrm{C}\right)$ & $\boldsymbol{T}_{r}\left({ }^{\circ} \mathrm{C}\right)$ & $T_{s}\left({ }^{\circ} \mathrm{C}\right)$ \\
\hline \multirow{6}{*}{1} & \multirow{6}{*}{200} & ABAQUS & 108.13 & 280.64 & 362.04 & 426.20 \\
\hline & & TOAST 1 & 106.61 & 277.75 & 359.26 & 421.65 \\
\hline & & TOAST 2 & 109.87 & 280.29 & 361.83 & 424.07 \\
\hline & & TOAST 3 & 102.30 & 274.39 & 355.85 & 418.45 \\
\hline & & TOAST 4 & 105.68 & 277.03 & 358.52 & 420.96 \\
\hline & & TOAST 5 & 105.25 & 276.69 & 358.18 & 420.64 \\
\hline \multirow{6}{*}{2} & \multirow{6}{*}{225} & ABAQUS & 113.30 & 303.13 & 395.55 & 465.08 \\
\hline & & TOAST 1 & 113.35 & 301.49 & 393.75 & 461.15 \\
\hline & & TOAST 2 & 116.90 & 304.21 & 396.53 & 463.75 \\
\hline & & TOAST 3 & 108.57 & 297.84 & 390.03 & 457.68 \\
\hline & & TOAST 4 & 112.33 & 300.71 & 392.96 & 460.41 \\
\hline & & TOAST 5 & 112.07 & 300.51 & 392.76 & 460.22 \\
\hline \multirow{6}{*}{3} & \multirow{6}{*}{250} & ABAQUS & 118.43 & 325.65 & 428.57 & 503.05 \\
\hline & & TOAST 1 & 120.02 & 324.52 & 427.77 & 499.79 \\
\hline & & TOAST 2 & 123.86 & 327.41 & 430.74 & 502.55 \\
\hline & & TOAST 3 & 114.80 & 320.60 & 423.75 & 496.04 \\
\hline & & TOAST 4 & 118.92 & 323.69 & 426.92 & 498.99 \\
\hline & & TOAST 5 & 118.88 & 323.66 & 426.90 & 498.97 \\
\hline \multirow{6}{*}{4} & \multirow{6}{*}{275} & ABAQUS & 123.52 & 347.25 & 461.30 & 540.37 \\
\hline & & TOAST 1 & 126.66 & 346.92 & 461.43 & 537.69 \\
\hline & & TOAST 2 & 130.77 & 349.97 & 464.57 & 540.62 \\
\hline & & TOAST 3 & 121.00 & 342.74 & 457.11 & 533.68 \\
\hline & & TOAST 4 & 125.48 & 346.05 & 460.53 & 536.85 \\
\hline & & TOAST 5 & 125.72 & 346.23 & 460.71 & 537.03 \\
\hline \multirow{6}{*}{5} & \multirow{6}{*}{300} & ABAQUS & 128.57 & 368.31 & 493.73 & 577.06 \\
\hline & & TOAST 1 & 133.06 & 368.61 & 494.63 & 574.83 \\
\hline & & TOAST 2 & 137.31 & 371.71 & 497.86 & 577.82 \\
\hline & & TOAST 3 & 127.05 & 364.23 & 490.09 & 570.62 \\
\hline & & TOAST 4 & 131.84 & 367.71 & 493.71 & 573.97 \\
\hline & & TOAST 5 & 132.71 & 368.35 & 494.37 & 574.58 \\
\hline
\end{tabular}


The same trends that are observable for the constant conductivity benchmarks can be seen in the variable conductivity benchmarks with the notable exception of the strong variation in the temperature values when compared to the temperature values of the constant conductivity benchmarks. This indicates that this sort-of thermal analysis problem is highly sensitive to changes in the thermal conductivity and thus allowing the user to input a variable conductivity equation in multiple formats (power or polynomial) is an added benefit of TOAST. This also demonstrates that in this problem (i.e., any irradiation thermal analysis), the temperatures are equally if not more sensitive to variations in the thermal conductivity relative to variations in the convection heat transfer coefficient.

Once again, the sensitivity of the temperatures to convection heat transfer accuracy is shown through the different $N u$ correlations. While similar trends are observable in Table 4 and Table 2, it should be noted that conduction (i.e., thermal conductivities) plays an even bigger role in the accuracy of the thermal analysis, per the comparison between the temperatures in Table 1 and Table 3.

Table 4. Percent difference between ABAQUS and TOAST for the variable conductivity benchmarks.

\begin{tabular}{|c|c|c|c|c|c|c|}
\hline Case & $\boldsymbol{q}_{\text {limit }}^{\prime}(W / \mathrm{cm})$ & Computation & $\epsilon_{c 1}$ & $\epsilon_{c 2, \text { out }}$ & $\epsilon_{r}$ & $\epsilon_{s}$ \\
\hline \multirow{5}{*}{1} & \multirow{5}{*}{200} & TOAST 1 & $-1.40 \%$ & $-1.03 \%$ & $-0.77 \%$ & $-1.07 \%$ \\
\hline & & TOAST 2 & $1.61 \%$ & $-0.12 \%$ & $-0.06 \%$ & $-0.50 \%$ \\
\hline & & TOAST 3 & $-5.40 \%$ & $-2.23 \%$ & $-1.71 \%$ & $-1.82 \%$ \\
\hline & & TOAST 4 & $-2.26 \%$ & $-1.29 \%$ & $-0.97 \%$ & $-1.23 \%$ \\
\hline & & TOAST 5 & $-2.66 \%$ & $-1.41 \%$ & $-1.07 \%$ & $-1.31 \%$ \\
\hline \multirow{5}{*}{2} & \multirow{5}{*}{225} & TOAST 1 & $0.04 \%$ & $-0.54 \%$ & $-0.45 \%$ & $-0.84 \%$ \\
\hline & & TOAST 2 & $3.18 \%$ & $0.36 \%$ & $0.25 \%$ & $-0.29 \%$ \\
\hline & & TOAST 3 & $-4.17 \%$ & $-1.75 \%$ & $-1.39 \%$ & $-1.59 \%$ \\
\hline & & TOAST 4 & $-0.86 \%$ & $-0.80 \%$ & $-0.66 \%$ & $-1.00 \%$ \\
\hline & & TOAST 5 & $-1.08 \%$ & $-0.86 \%$ & $-0.71 \%$ & $-1.04 \%$ \\
\hline \multirow{5}{*}{3} & \multirow{5}{*}{250} & TOAST 1 & $1.34 \%$ & $-0.35 \%$ & $-0.19 \%$ & $-0.65 \%$ \\
\hline & & TOAST 2 & $4.58 \%$ & $0.54 \%$ & $0.51 \%$ & $-0.10 \%$ \\
\hline & & TOAST 3 & $-3.07 \%$ & $-1.55 \%$ & $-1.12 \%$ & $-1.39 \%$ \\
\hline & & TOAST 4 & $0.41 \%$ & $-0.60 \%$ & $-0.38 \%$ & $-0.81 \%$ \\
\hline & & TOAST 5 & $0.38 \%$ & $-0.61 \%$ & $-0.39 \%$ & $-0.81 \%$ \\
\hline \multirow{5}{*}{4} & \multirow{5}{*}{275} & TOAST 1 & $2.54 \%$ & $-0.09 \%$ & $0.03 \%$ & $-0.50 \%$ \\
\hline & & TOAST 2 & $5.87 \%$ & $0.78 \%$ & $0.71 \%$ & $0.05 \%$ \\
\hline & & TOAST 3 & $-2.04 \%$ & $-1.30 \%$ & $-0.91 \%$ & $-1.24 \%$ \\
\hline & & TOAST 4 & $1.58 \%$ & $-0.35 \%$ & $-0.17 \%$ & $-0.65 \%$ \\
\hline & & TOAST 5 & $1.78 \%$ & $-0.29 \%$ & $-0.13 \%$ & $-0.62 \%$ \\
\hline \multirow{5}{*}{5} & \multirow{5}{*}{300} & TOAST 1 & $3.49 \%$ & $0.08 \%$ & $0.18 \%$ & $-0.39 \%$ \\
\hline & & TOAST 2 & $6.80 \%$ & $0.92 \%$ & $0.84 \%$ & $0.13 \%$ \\
\hline & & TOAST 3 & $-1.18 \%$ & $-1.11 \%$ & $-0.74 \%$ & $-1.12 \%$ \\
\hline & & TOAST 4 & $2.54 \%$ & $-0.16 \%$ & $0.00 \%$ & $-0.53 \%$ \\
\hline & & TOAST 5 & $3.22 \%$ & $0.01 \%$ & $0.13 \%$ & $-0.43 \%$ \\
\hline
\end{tabular}

Nonetheless, it is once again astonishing how accurate the centerline sample temperatures are predicted by TOAST (relative to ABAQUS), where $\epsilon<2 \%$ at the most. 


\section{CONCLUSIONS}

A Thermal-Hydraulic Optimization, Analysis, and Scoping Tool (referred to as TOAST) was successfully created for easier programmatic, optimization, and sensitivity analyses, particularly towards helping fulfil the mission of the NMDQi program. TOAST was built on a semi-2D thermal analysis framework where thermal resistance networks are solved radially at multiple axial locations until the axial and radial centerline is reached, at which symmetry conditions are assumed, enabling a significant reduction in the computational expense. It resembles a steady-state 3D-axissymmetric simulation with a mesh that consists of only five radial nodes and a user-supplied number axial node (100-1000 nodes per the user's preferences). The discretization scheme adopted in TOAST allows for a more accurate calculation of the coolant temperature at different axial heights, resembling a backward difference discretization scheme. It was found that 500 axial nodes were sufficient to obtain reasonably accurate results with less than a $2 \%$ difference to results from a 3D-axissymmetric simulation in ABAQUS software.

TOAST is driven by user-supplied inputs in a highly accessible GUI that requires little-to-no thermalhydraulic experience from the user. The GUI allows the user to perform thermal-hydraulic computations for the axial temperature profiles of each component in a cylindrical irradiation vehicle by calculating the coolant temperature alongside the temperatures of any number of capsules used, the rodlet cladding surrounding the sample, and the cylindrical sample itself. The user can supply temperature limits for the capsules and sample or can provide a sample heat rate (constant or cosine-shaped) in order to obtain the thermal behavior. If the user does not supply a heat rate for the sample, the allowable sample heat rate will be displayed on the GUI upon its computation. The user is also able to leave certain dimensions or temperature limits as unknowns, and TOAST will be able to produce plots for the sensitivity of the sample heat rate, the maximum coolant temperature, and the ASME pressure limits of the capsules with respect to changes in the unknown parameter. This allows the user to perform sensitivity studies that would require multiple meshes and lengthy simulations in other codes anywhere between seconds and minutes (depending on the local machine's performance).

A benchmark was carried out to compare the performance of TOAST against the ABAQUS commercial software, which is highly utilized in INL for thermal-hydraulic safety and programmatic analyses. The results show that TOAST is within less than $3 \%$ of ABAQUS in most cases and a maximum of $8 \%$ difference in some computations. The benchmarks showed that this uncertainty is tied back to the appropriate selection of a Nusselt number correlation or the appropriate selection of thermal conductivity values (whether constant or variable). Nonetheless, for the purposes of programmatic and sensitivity analyses, it is a great tool due to its computational cost efficiency, its high accessibility, and its sensitivity analysis features. 


\section{Appendix: TOAST MATLAB Program}

Each appendix subsection contains a MATLAB function or txt file that is necessary to run TOAST. Each of the 10 files (nine.$m$ files and at least one .txt file) must be stored in the same directory. Once all the files are stored in the same directory, the TOAST GUI can be called by either running the TOAST_GUI.m file in MATLAB or typing TOAST_GUI in the MATLAB command window. Once the GUI is displayed, the user can use TOAST to its full capability. It should be noted that a lot of GUI elements (including all the buttons) can show a callout message when the user hovers their cursor over them, providing insight into the functions and limitations of each button (or some other GUI elements).

It should also be noted that, for all the sensitivity study features to work, within the same directory, the following subdirectories must be created manually and named exactly as they are named below. The SampleStats and tBondStats subdirectories are only necessary for studying the sensitivity with respect to an unknown sample diameter and an unknown bond thickness (respectively).

- Coolant_Max_Temps

- Heat_and_Pressure_Limits

- SampleStats

- tBondStats 


\subsection{TOAST_GUI.m}

function TOAST_GUI

\%Tabl Elements

global Tc1_max_Label Tc2_max_Label Tinf_max_Label q_Label Stat_Go2_Label... Stat_Lābel Q_PRIME tèxt_Tinf_in text_DP text_Piñf DISCs BoñdChēck...

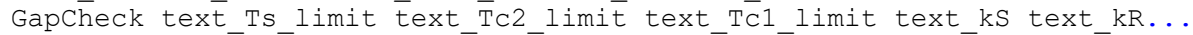

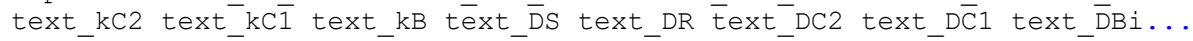
text_DBo text_LS text_L̄C text_L̄B axG axT FSize- NuCor_Chēck

oTab2 Elēments

global QCos Check $F$ kBond E kBond D kBond C kBond B kBond A kBond...

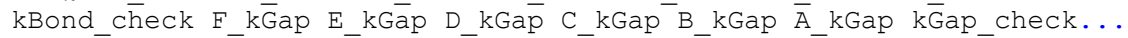

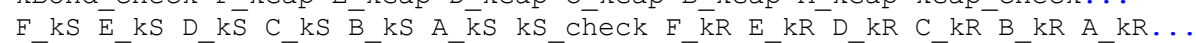
$\mathrm{k} \overline{\mathrm{R}}$ che $\bar{c} \mathrm{k} \overline{\mathrm{k}} \mathrm{C} 2 \mathrm{E}^{-} \mathrm{kC} 2 \overline{\mathrm{D}}_{\mathrm{kC}}{ }^{-} \mathrm{C} \mathrm{kC}^{-}{ }^{-} \mathrm{B} \mathrm{kC} 2 \mathrm{~A}^{-} \mathrm{kC} 2 \overline{\mathrm{k}} \mathrm{C} 2$ check $\overline{\mathrm{F}} \mathrm{kC1}{ }^{-} \mathrm{E} k \mathrm{k} \overline{1} \ldots$

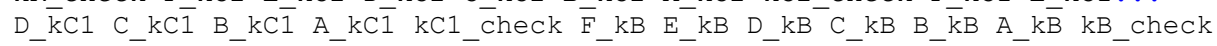

close all

ClC

MAINFIG=figure('Name', 'TOAST (V.1) ', 'NumberTitle',...

'off', 'units', 'pixels', 'Position', [88 67 1224 597]);

TAGR=uitabgroup (MAINFIG);

TABS (1)=uitab (TAGR, 'Title', 'Main Settings') ;

TABS (2)=uitab (TAGR, 'Title', 'Additional Settings') ;

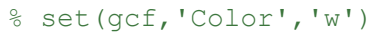

$\mathrm{X} 0=0.01 ; \mathrm{Y} 0=0.95 ; \mathrm{X} 1=0.1 ; \mathrm{Y} 1=0.035 ;$

$\mathrm{DY}=0.05 ; \mathrm{DX}=0.275$;

FSize=10;

\% 응 Heights

numy=0; numx $=0$;

uicontrol (TABS (1), 'Style', 'text', 'String', 'Heights', 'units', 'normalized',...

'Position', [X0+(DX*numx) +0.11 Y0-(DY*numy) X1 Y1], 'FontWeight', 'bold', 'FontSize', FSize+1) ;

numy=numy+1;

uicontrol (TABS (1), 'Style', 'text', 'String', 'Basket', 'units', 'normalized',...

'Position', [X0+(DX*numx) Y0-(DY*numy)'X1 Y1],'FontWeight', 'bold', 'FontSize', FSize); text $\mathrm{LB}=$

uicoñtrol (TABS (1), 'Style', 'edit', 'String', '1.2192', 'units', 'normalized', 'Position', [X0+(DX*numx)+ $0.11 \mathrm{Y0}-(\mathrm{DY}$ *numy) X1 Y1]);

uicontrol (TABS (1), 'Style', 'text', 'String', 'm', 'units', 'normalized',...

'Position', [X0+(DX*numx)+0.21 Y0-(DY*numy) Y1 Y1],'FontSize',FSize) ;

numy=numy+1;

uicontrol (TABS(1), 'Style', 'text', 'String', 'Capsules \& Rodlet', 'units', 'normalized',...

'Position', [X0+(DX*numx) YO-(DY*numy) X1 Y1],'FontWeight','bold', 'FontSize', FSize); text $\mathrm{LC}=$

uicoñtrol (TABS (1), 'Style', 'edit', 'String', '0.1778', 'units', 'normalized', 'Position', [X0+(DX*numx)+ $0.11 \mathrm{Y} 0-\left(\mathrm{DY}{ }^{*}\right.$ numy) $\left.\left.\mathrm{X} 1 \mathrm{Y} 1\right]\right)$;

uicontrol (TABS (1), 'Style', 'text', 'String', 'm', 'units', 'normalized',...

'Position', [X0+(DX*numx)+0.21 Y0-(DY*numy) Y1 Y1],'FontSize',FSize) ;

numy=numy +1 ;

uicontrol (TABS (1), 'Style', 'text', 'String', 'Sample', 'units', 'normalized',...

'Position', [X0+(DX*numx) Y0-(DY*numy) X1 Y1],'FontWeight', 'bold', 'FontSize', FSize);

text LS =

uicoñtrol (TABS (1), 'Style', 'edit', 'String', '0.02', 'units', 'normalized', 'Position', [x0+(DX*numx)+0.

$11 \mathrm{Y} 0-\left(\mathrm{DY}{ }^{*}\right.$ numy) X1 Y1]);

uicontrol (TABS (1), 'Style', 'text', 'String', 'm', 'units', 'normalized',...

'Position', [X0+(DX*numx)+0.21 Y0-(DY*numy) Y1 Y1],'FontSize',FSize);

\% 응 Diameters

numy=numy+1; numx=0;

uicontrol (TABS (1), 'Style', 'text', 'String', 'Diameters', 'units', 'normalized',...

'Position', [X0+(DX*numx)+0.11 Y0-(DY*numy) X1 Y1],'FontWeight', 'bold', 'FontSize', FSize+1);

numy=numy+1;

uicontrol (TABS (1), 'Style', 'text', 'String', 'Basket OD', 'units', 'normalized',...

'Position', [X0+(DX*numx) Y0-(DY*numy) X1 Y1],'FontWeight', 'bold', 'FontSize',FSize); 
text $\mathrm{DBO}=$

uicontrol (TABS (1), 'Style', 'edit', 'String', '0.0159', 'units', 'normalized', 'Position', [X0+(DX*numx)+ $0.11 \mathrm{Y} 0-\left(\mathrm{DY}{ }^{*}\right.$ numy) $\left.\left.\mathrm{X} 1 \mathrm{Y} 1\right]\right)$;

uicontrol (TABS (1), 'Style', 'text', 'String', 'm', 'units', 'normalized',...

'Position', [X0+(DX*numx)+0.21 Y0-(DY*numy) Y1 Y1], 'FontSize', FSize);

numy=numy+1;

uicontrol (TABS (1), 'Style', 'text', 'String', 'Basket ID', 'units', 'normalized',...

'Position', [X0+(DX*numx) Y0-(DY*numy) X1 Y1],'FontWeight','bold', 'FontSize',FSize);

text $\mathrm{DBi}=$

uicoñtrol (TABS (1), 'Style', 'edit', 'String', '0.0152', 'units', 'normalized', 'Position', [x0+(DX*numx)+

$0.11 \mathrm{YO}-\left(\mathrm{DY}{ }^{*}\right.$ numy) $\left.\left.\mathrm{X} 1 \mathrm{Y} 1\right]\right)$;

uicontrol (TABS (1), 'Style', 'text', 'String', 'm', 'units', 'normalized',...

'Position', [X0+(DX*numx) +0.21 Y0-(DY*numy) Y1 Y1],' FontSize', FSize);

numy=numy+1;

uicontrol (TABS (1), 'Style', 'text', 'String', 'Capsule 1 OD', 'units', 'normalized',...

'Position', [X0+(DX*numx) Y0-(DY*numy) X1 Y1],'FontWeight', 'bold', 'FontSize', FSize) ; text $\mathrm{DC} 1=$

uicoñtrol (TABS (1), 'Style', 'edit', 'String', '0.0124', 'units', 'normalized', 'Position', [Xo+(DX*numx)+ $0.11 \mathrm{Y} 0-\left(\mathrm{DY}{ }^{*}\right.$ numy) $\left.\left.\mathrm{X} 1 \mathrm{Y} 1\right]\right)$;

uicontrol (TABS (1), 'Style', 'text', 'String', 'm', 'units', 'normalized',...

'Position', [X0+(DX*numx) +0.21 Y0-(DY*numy) Y1 Y1],'FontSize',FSize);

numy=numy+1;

uicontrol (TABS(1), 'Style', 'text', 'String', 'Capsule 2 OD', 'units', 'normalized',...

'Position', [X0+(DX*numx) Y0-(DY*numy) X1 Y1],'FontWeight','bold', 'FontSize', FSize);

text $\mathrm{DC} 2=$

uicoñtrol (TABS (1), 'Style', 'edit', 'String', '0.0116', 'units', 'normalized', 'Position', [X0+(DX*numx)+

$0.11 \mathrm{Y} 0-\left(\mathrm{DY}{ }^{*}\right.$ numy) $\left.\left.\mathrm{X} 1 \mathrm{Y} 1\right]\right)$;

uicontrol (TABS (1), 'Style', 'text', 'String', 'm', 'units', 'normalized',...

'Position', [X0+(DX*numx) +0.21 Y0-(DY*numy) Y1 Y1], 'FontSize', FSize);

numy=numy+1;

uicontrol (TABS (1), 'Style', 'text', 'String', 'Rodlet OD', 'units', 'normalized',...

'Position', [X0+(DX*numx) Y0-(DY*numy) X1 Y1],'FontWeight','bold', 'FontSize',FSize);

text $\mathrm{DR}=$

uicontrol (TABS (1), 'style', 'edit', 'String', '0.0098', 'units', 'normalized', 'Position', [X0+(DX*numx)+

$0.11 \mathrm{Y} 0-(\mathrm{DY} *$ numy) $\mathrm{X} 1 \mathrm{Y} 1])$;

uicontrol (TABS (1), 'Style', 'text', 'String', 'm', 'units', 'normalized',...

'Position', [X0+(DX*numx)+0.21 Y0-(DY*numy) Y1 Y1], 'FontSize',FSize);

numy=numy+1;

uicontrol (TABS (1), 'Style', 'text', 'String', 'Sample', 'units', 'normalized',...

'Position', [X0+(DX*numx) Y0-(DY*numy)'X1 Y1],'FontWeight', 'bold', 'FontSize', FSize);

text $\mathrm{DS}=$

uicontrol (TABS (1), 'Style', 'edit', 'String', '0.0083', 'units', 'normalized', 'Position', [X0+(DX*numx)+

$0.11 \mathrm{Y} 0-\left(\mathrm{DY}{ }^{\star}\right.$ numy) $\left.\left.\mathrm{X} 1 \mathrm{Y} 1\right]\right)$;

uicontrol (TABS (1), 'Style', 'text', 'String', 'm', 'units', 'normalized',...

'Position', [X0+(DX*numx)+0.21 Y0-(DY*numy) Y1 Y1],'FontSize',FSize);

\% \% Conductivities

numy=numy+1; numx=0;

uicontrol (TABS (1), 'Style', 'text', 'String', 'Conductivities', 'units', 'normalized', ...

'Position', [X0+(DX*numx)+0.11 Y0-(DY*numy) X1 Y1],'FontWeight', 'bold', 'FontSize',FSize+1);

numy=numy+1;

uicontrol (TABS (1), 'Style', 'text', 'String', 'Basket (unused) ', 'units', 'normalized',...

'Position', [X0+(DX*numx) Y0-(DY*numy) X1 Y1],'FontWeight', 'bold', 'FontSize',FSize) ;

text_kB $=$

uicoñtrol (TABS (1), 'Style', 'edit', 'String', '15', 'units', 'normalized', 'Position', [X0+(DX*numx) +0.11

$\mathrm{Y} 0-\left(\mathrm{DY}{ }^{\star}\right.$ numy) $\left.\left.\mathrm{X} 1 \mathrm{Y} 1\right]\right)$;

uicontrol (TABS (1), 'Style', 'text', 'String', 'W/m-K', 'units', 'normalized',...

'Position', [X0+(DX*numx)+0.21 Y0-(DY*numy) Y1+0.01 Y1],'FontSize',FSize);

numy=numy+1;

uicontrol (TABS (1), 'Style', 'text', 'String', 'Capsule 1', 'units', 'normalized',...

'Position', [X0+(DX*numx) Y0-(DY*numy) X1 Y1],'FontWeight','bold', 'FontSize', FSize); text_kC1

uicoñtrol (TABS (1), 'Style', 'edit', 'String', '18.9', 'units', 'normalized', 'Position', [Xo+(DX*numx)+0.

$11 \mathrm{Y} 0-\left(\mathrm{DY}{ }^{*}\right.$ numy) $\left.\left.\mathrm{X} 1 \mathrm{Y} 1\right]\right)$; 
uicontrol (TABS (1), 'Style', 'text', 'String', 'W/m-K', 'units', 'normalized',...

'Position', [X0+(DX*numx)+0.21 Y0-(DY*numy) Y1+0.01 Y1],'FontSize',FSize);

numy=numy+1;

uicontrol (TABS (1), 'Style', 'text', 'String', 'Capsule 2', 'units', 'normalized',...

'Position', [X0+(DX*numx) Y0-(DY*numy) X1 Y1],'FontWeight','bold','FontSize',FSize);

text_kC2 =

uicoñtrol (TABS (1), 'Style', 'edit', 'String', '18.9', 'units', 'normalized', 'Position', [Xo+(DX*numx)+0.

$11 \mathrm{Y} 0-\left(\mathrm{DY}{ }^{\star}\right.$ numy) $\left.\left.\mathrm{X} 1 \mathrm{Y} 1\right]\right)$;

uicontrol (TABS (1), 'Style','text', 'String', 'W/m-K', 'units', 'normalized',...

'Position', [X0+(DX*numx)+0.21 Y0-(DY*numy) Y1+0.01 Y1],'FontSize',FSize);

numy=numy+1;

uicontrol (TABS (1), 'Style', 'text', 'String', 'Rodlet', 'units', 'normalized',...

'Position', [X0+(DX*numx) Y0-(DY*numy) X1 Y1],'FontWeight','bold', 'FontSize', FSize) ; text_kR =

uicontrol (TABS (1), 'style', 'edit', 'String', '25', 'units', 'normalized', 'Position', [x0+(DX*numx)+0.11

$\mathrm{YO}-\left(\mathrm{DY}{ }^{\star}\right.$ numy) $\left.\left.\mathrm{X} 1 \mathrm{Y} 1\right]\right)$;

uicontrol (TABS (1), 'Style', 'text', 'String', 'W/m-K', 'units', 'normalized',...

'Position', [X0+(DX*numx)+0.21 Y0-(DY*numy) Y1+0.01 Y1],'FontSize',FSize);

numy $=$ numy+1;

uicontrol (TABS (1), 'Style', 'text', 'String', 'Sample', 'units', 'normalized',...

'Position', [X0+(DX*numx) Y0-(DY*numy) X1 Y1],'FontWeight','bold', 'FontSize', FSize); text_kS =

uicontrol (TABS (1), 'Style', 'edit', 'String', '3.6', 'units', 'normalized', 'Position', [X0+(DX*numx)+0.1

$1 \mathrm{YO}-\left(\mathrm{DY}{ }^{\star}\right.$ numy) $\left.\left.\mathrm{X} 1 \mathrm{Y} 1\right]\right)$;

uicontrol (TABS (1), 'Style', 'text', 'String', 'W/m-K', 'units', 'normalized',...

'Position', [X0+(DX*numx) +0.21 Y0-(DY*numy) Y1+0.01 Y1],'FontSize', FSize);

\% $\frac{\circ}{\circ}$ Temperature Limits

numy=0; numx=1;

uicontrol (TABS (1), 'Style', 'text', 'String', 'Temperature Limits', 'units', 'normalized', .. 'Position', [X0+(DX*numx) $+0.1^{\prime} \mathrm{Y} 0-\left(\mathrm{DY} \mathrm{DX}^{\star} \mathrm{numy}\right) \mathrm{X} 1+0.02$

Y1], 'FontWeight', 'bold', 'FontSize', FSize+1);

numy=numy+1;

uicontrol (TABS (1), 'Style', 'text', 'String', 'Capsule 1', 'units', 'normalized',...

'Position', [X0+(DX*numx) Y0-(DY*numy) X1 Y1],'FontWeight','bold','FontSize',FSize);

text_Tc1_limit $=$

uicontrō̄ (TABS (1), 'Style', 'edit', 'String', '500', 'units', 'normalized', 'Position', [X0+(DX*numx)+0.1

$1 \mathrm{YO}-\left(\mathrm{DY}{ }^{\star}\right.$ numy) $\left.\left.\mathrm{X} 1 \mathrm{Y} 1\right]\right)$;

uicontrol (TABS (1), 'Style', 'text', 'String', 'C', 'units', 'normalized',...

'Position', [X0+(DX*numx)+0.21 Y0-(DY*numy) Y1+0.01 Y1],'FontSize',FSize);

numy=numy+1;

uicontrol (TABS (1), 'Style', 'text', 'String', 'Capsule 2', 'units', 'normalized',...

'Position', [X0+(DX*numx) Y0-(DY*numy) X1 Y1],'FontWeight','bold', 'FontSize',FSize);

text_Tc2_limit $=$

uicoñtrō̄ (TABS (1), 'Style', 'edit', 'String', '500', 'units', 'normalized', 'Position', [X0+(DX*numx) +0.1

$1 \mathrm{Y} 0-(\mathrm{DY}$ numy) $\mathrm{X} 1 \mathrm{Y} 1])$;

uicontrol (TABS (1), 'Style', 'text', 'String', 'C', 'units', 'normalized',...

'Position', [X0+(DX*numx)+0.21 Y0-(DY*numy) Y1+0.01 Y1],'FontSize',FSize);

numy=numy+1;

uicontrol (TABS (1), 'Style', 'text', 'String', 'Sample', 'units', 'normalized',...

'Position', [X0+(DX*numx) Y0-(DY*numy) X1 Y1],'FontWeight','bold', 'FontSize',FSize).

text_Ts_limit $=$

uicoñtrōl (TABS (1), 'Style', 'edit', 'String', '1300', 'units', 'normalized', 'Position', [X0+(DX*numx) +0.

$11 \mathrm{Y} 0-\left(\mathrm{DY}{ }^{\star}\right.$ numy) X1 Y1]);

uicontrol (TABS (1), 'Style', 'text', 'String', 'C', 'units', 'normalized',...

'Position', [X0+(DX*numx)+0.21 Y0-(DY*numy) Y1+0.01 Y1],'FontSize',FSize);

\%options row

numy=numy+1;

uicontrol (TABS (1), 'Style', 'text', 'String', 'Options', 'units', 'normalized',...

'Position', [X0+(DX*numx) Y0-(DY*numy) X1 Y1],'FontWeight','bold', 'FontSize',FSize);

uicontrol (TABS(1), 'Style', 'text', 'String', 'Gap thickness, bond thickness, and the

discretization', 'units', 'normalized',...

'Position', [X0+(DX*numx) Y0-(DY*numy)-0.03 X1 Y1], 'FontWeight', 'bold', 'FontSize',6);

응 Gap Check 
hintl=['Assumes gas gap filled with Helium and 20\% argon fraction, with the set thickness [m] (can be zero)', newline, 'Located between the capsules 1 and 2', newline,... ' $\left.\mathrm{k}(\mathrm{T})=0.00020347396 * \mathrm{~T}+0.0423552^{\prime}\right]$;

Gapcheck = uicontrol(TABS(1), 'Style', 'edit', 'String', '1E-5', 'units', 'normalized', ... 'Position', [X0+(DX*numx)+0.11 Y0-(DY*numy) X1/2 Y1], 'Tooltipstring', hint1);

응 Thermal bond thickness

hintl=['Assumes liquid sodium bond with the set thickness [m] (can be zero)', newline, 'Located between capsule 2 and the rodlet', newline,... $\mathrm{\prime} k(\mathrm{~T})=111.5731-(0.07026424 * \mathrm{~T})+\left(\mathrm{T}^{\wedge} 2 * 2.373302 \mathrm{E}-5\right)-\left(\mathrm{T}^{\wedge} 3 * 8.567 \mathrm{E}-9\right)+\left(\mathrm{T}^{\wedge} 4 * 2.025858 \mathrm{E}-12\right)-\left(\mathrm{T}^{\wedge} 5\right.$ * 2.163437E-16)']; 


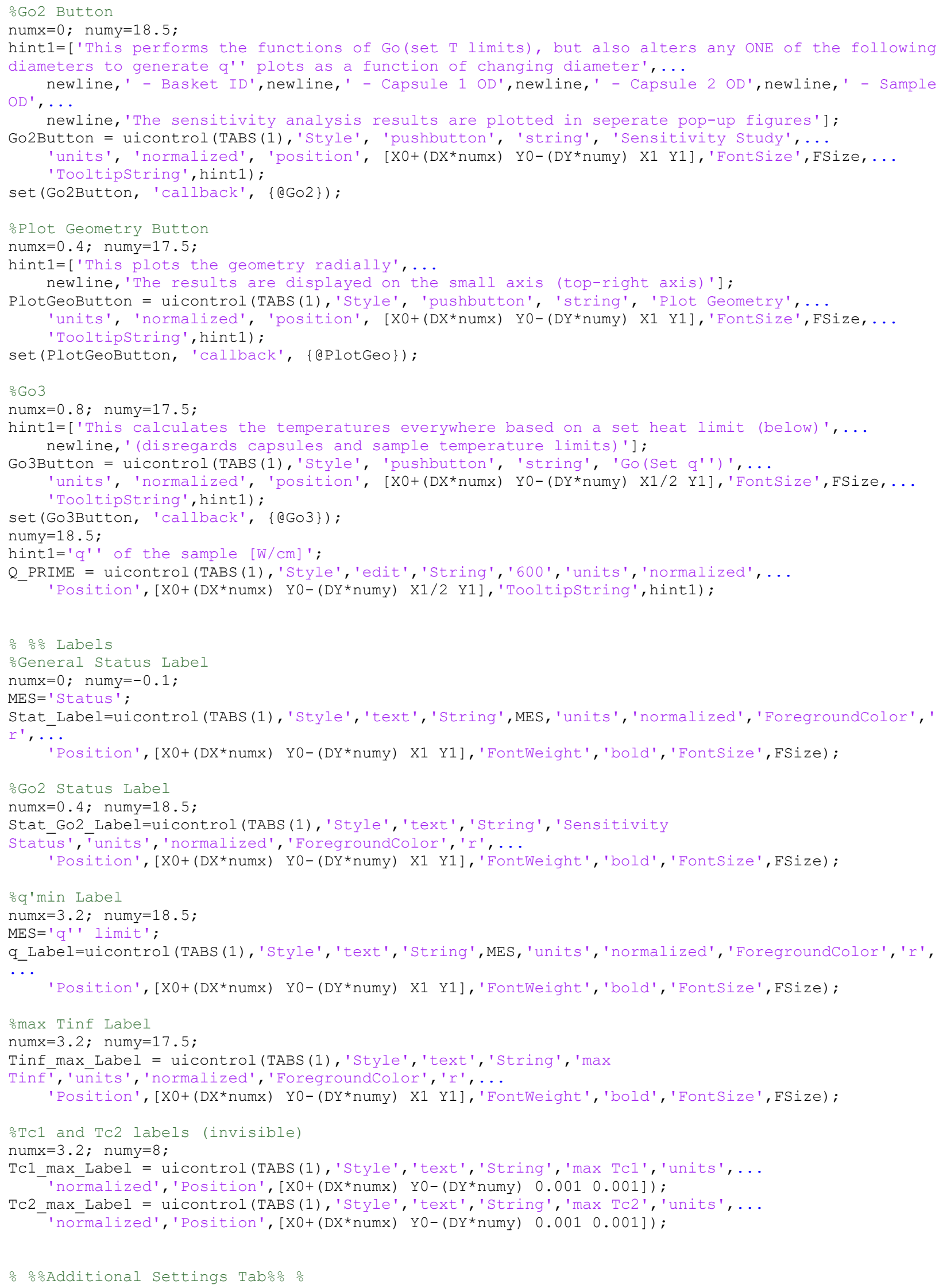


$\mathrm{X} 0=0.01 ; \mathrm{Y} 0=0.95 ; \mathrm{X} 1=0.1 ; \mathrm{Y} 1=0.035 ;$

$\mathrm{DY}=0.05 ; \mathrm{DX}=0.275$;

FSize=10;

응 Conductivities Additional Settings numy=0; numx $=0$;

uicontrol(TABS(2), 'Style', 'text', 'string', 'Conductivities as a function of temperature', 'units', 'normalized',...

'Position', [X0+(DX*numx)+0.005 Y0-(DY*numy*0.5) X1*3

Y1], 'FontWeight', 'bold', 'FontSize', FSize+1, 'HorizontalAlignment', 'left');

uicontrol (TABS (2), 'Style', 'text', 'String', 'Format', 'units', 'normalized',...

'Position', [X0+(DX*numx) +0.12 Y0-(DY*(numy+0.8)) X1 Y1],'FontSize', FSize);

numy=numy +1.5 ;

uicontrol (TABS (2), 'Style', 'text', 'String', 'Basket (unused) ', 'units', 'normalized',...

'Position', [X0+(DX*numx) Y0-(DY*numy) X1 Y1],'FontWeight','bold', 'FontSize',FSize); $\mathrm{kB}$ check $=$

uic̄ontrol (TABS (2), 'Style', 'popupmenu', 'String', \{'Constant ', 'Polynomial', 'Power'\}, 'units', 'normali zed',...

'Position', $\left[\mathrm{X} 0+\left(\mathrm{DX} \mathrm{X}^{\star} \mathrm{numx}\right)+0.12 \mathrm{Y} 0-(\mathrm{DY} *\right.$ numy $) \mathrm{X} 1$

Y1], 'FontSize', FSize, 'Callback', (akBCHECKER) ;

A_kB_lab=uicontrol (TABS (2), 'Style', 'text', 'String', 'po', 'units', 'normalized',...

'Position', [X0+(DX*0)+0.23 Y0-(DY*(numy-0.8)) X1 Y1], 'FontSize', FSize, 'Visible', 'off');

A_kB_lab2=uicontrol (TABS (2), 'Style', 'text','String', 'A', 'units', 'normalized',...

'Position', [X0+(DX*0)+0.23 Y0-(DY*(numy-0.8)) X1 Y1],'FontSize', FSize,'Visible', 'off');

A kB=uicontrol (TABS (2), 'Style', 'edit', 'String', 1, 'units', 'normalized',...

'Position', [X0+(DX*0)+0.23 Y0-(DY*numy) X1 Y1], 'FontSize',FSize, 'Visible', 'off');

B kB lab=uicontrol (TABS (2), 'Style', 'text', 'String', 'pl', 'units', 'normalized',...

'Position', [X0+(DX*0)+0.34 Y0-(DY*(numy-0.8)) X1 Y1], 'FontSize', FSize, 'Visible', 'off');

B kB lab2=uicontrol (TABS (2), 'Style', 'text', 'String', 'B', 'units', 'normalized',...

'Position', [X0+(DX*0)+0.34 Y0-(DY*(numy-0.8)) X1 Y1],'FontSize', FSize, 'Visible', 'off');

B_kB=uicontrol(TABS (2), 'Style', 'edit', 'String', 1, 'units', 'normalized',...

'Position', [X0+(DX*0)+0.34 Y0-(DY*numy) X1 Y1],'FontSize',FSize, 'Visible', 'off');

C_kB_lab=uicontrol(TABS (2), 'Style',' 'text',' String', 'p2', 'units', 'normalized',...

'Position', [X0+(DX*0)+0.45 Y0-(DY*(numy-0.8)) X1 Y1],'FontSize', FSize, 'Visible', 'off');

C_kB_lab2=uicontrol (TABS (2), 'Style', 'text', 'String', 'C', 'units', 'normalized',...

'Position', [X0+(DX*0)+0.45 Y0-(DY*(numy-0.8)) X1 Y1], 'FontSize', FSize,'Visible', 'off');

C_kB=uicontrol (TABS (2), 'Style', 'edit', 'String', 1, 'units', 'normalized',...

'Position', [X0+(DX*0)+0.45 Y0-(DY*numy) X1 Y1],'FontSize',FSize,'Visible', 'off');

D_kB_lab=uicontrol (TABS (2), 'Style', 'text', 'String', 'p3', 'units', 'normalized',...

'Position', [X0+(DX*0)+0.56 Y0-(DY*(numy-0.8)) X1 Y1],'FontSize', FSize, 'Visible', 'off');

D_kB=uicontrol (TABS (2), 'Style', 'edit', 'String', 1, 'units', 'normalized',...

'Position', [X0+(DX*0)+0.56 Y0-(DY*numy) X1 Y1],'FontSize',FSize,'Visible', 'off');

E_kB_lab=uicontrol (TABS (2), 'Style', 'text', 'String', 'p4', 'units', 'normalized',...

'Position', [X0+(DX*0)+0.67 Y0-(DY*(numy-0.8)) X1 Y1],'FontSize', FSize, 'Visible', 'off');

E kB=uicontrol(TABS (2), 'Style', 'edit', 'String', 1, 'units', 'normalized',...

'Position', [X0+(DX*0)+0.67 Y0-(DY*numy) X1 Y1],'FontSize',FSize,'Visible', 'off');

F kB lab=uicontrol(TABS (2), 'Style',' text','String', 'p5', 'units', 'normalized',...

'Position', [X0+(DX*0)+0.78 Y0-(DY* (numy-0.8)) X1 Y1],'FontSize', FSize, 'Visible', 'off');

F kB=uicontrol (TABS (2), 'Style', 'edit', 'String', 1, 'units', 'normalized',...

'Position', [X0+(DX*0)+0.78 Y0-(DY*numy) X1 Y1],'FontSize',FSize, 'Visible', 'off');

numy=numy+2;

uicontrol (TABS (2), 'Style', 'text', 'String', 'Capsule 1', 'units', 'normalized',...

'Position', [X0+(DX*numx) Y0-(DY*numy) X1 Y1],'FontWeight', 'bold', 'FontSize', FSize);

$\mathrm{kCl}$ _check $=$

uicontrol (TABS (2), 'Style', 'popupmenu', 'String', \{'Constant', 'Polynomial', 'Power'\}, 'units', 'normali zed',...

'Position', $\left[\mathrm{X} 0+\left(\mathrm{DX} \mathrm{X}^{\star} \mathrm{numx}\right)+0.12 \mathrm{Y} 0-(\mathrm{DY} *\right.$ numy) $\mathrm{X} 1$

Y1], 'FontSize', FSize, 'Callback', (akC1CHECKER);

A kC1 lab=uicontrol(TABS (2), 'Style', 'text', 'String', 'po', 'units', 'normalized',...

'Position', [X0+(DX*0)+0.23 Y0-(DY*(numy-0.8)) X1 Y1], 'FontSize', FSize, 'Visible', 'off') ;

A kC1 lab2=uicontrol (TABS (2), 'Style', 'text', 'String', 'A', 'units', 'normalized',...

'Position', [X0+(DX*0)+0.23 Y0-(DY*(numy-0.8)) X1 Y1],'FontSize',FSize, 'Visible', 'off');

A_kCl=uicontrol (TABS (2), 'Style', 'edit', 'String', 1, 'units', 'normalized',...

'Position', [X0+(DX*0)+0.23 Y0-(DY*numy) X1 Y1],'FontSize',FSize, 'Visible', 'off');

B_kC1_lab=uicontrol(TABS (2), 'style', 'text', 'String', 'pl', 'units', 'normalized', ...

'Position', [X0+(DX*0)+0.34 Y0-(DY*(numy-0.8)) X1 Y1],'FontSize', FSize, 'Visible', 'off');

B_kC1_lab2=uicontrol (TABS (2), 'Style', 'text', 'String ', 'B', 'units', 'normalized',...

'Position', [X0+(DX*0)+0.34 Y0-(DY*(numy-0.8)) X1 Y1],'FontSize',FSize, 'Visible', 'off');

B_kCl=uicontrol (TABS (2), 'Style', 'edit', 'String', 1, 'units', 'normalized',... 
'Position', [X0+(DX*0)+0.34 Y0-(DY*numy) X1 Y1],'FontSize',FSize, 'Visible', 'off');

C_kC1_lab=uicontrol (TABS (2), 'Style', 'text', 'String', 'p2', 'units', 'normalized', ...

'Position', [X0+(DX*0)+0.45 Y0-(DY*(numy-0.8)) X1 Y1], 'FontSize',FSize, 'Visible', 'off') ;

C kC1 lab2=uicontrol(TABS (2), 'Style', 'text', 'String', 'C', 'units', 'normalized',...

'Position', [X0+(DX*0)+0.45 Y0-(DY* (numy-0.8)) X1 Y1],'FontSize', FSize, 'Visible', 'off') ;

C kCl=uicontrol(TABS (2), 'Style', 'edit', 'String', 1, 'units', 'normalized',...

'Position', [X0+(DX*0)+0.45 Y0-(DY*numy) X1 Y1],'FontSize', FSize, 'Visible', 'off');

D kC1 lab=uicontrol (TABS (2), 'Style', 'text', 'String', 'p3', 'units', 'normalized',...

'Position', [X0+(DX*0)+0.56 Y0-(DY*(numy-0.8)) X1 Y1], 'FontSize', FSize, 'Visible', 'off');

D kCl=uicontrol (TABS (2),'style', 'edit','String', 1, 'units', 'normalized',...

'Position', [X0+(DX*0)+0.56 Y0-(DY*numy) X1 Y1],'FontSize',FSize, 'Visible', 'off');

E kCl lab=uicontrol (TABS (2), 'Style', 'text', 'String', 'p4', 'units', 'normalized', ..

'Position', [X0+(DX*0)+0.67 Y0-(DY* (numy-0.8)) X1 Y1],'FontSize', FSize, 'Visible', 'off') ;

E_kCl=uicontrol (TABS (2), 'Style', 'edit', 'String', 1, 'units', 'normalized',...

'Position', [X0+(DX*0)+0.67 Y0-(DY*numy) X1 Y1],'FontSize',FSize, 'Visible', 'off');

F_kC1_lab=uicontrol(TABS (2), 'Style', 'text', 'String', 'p5', 'units', 'normalized', ...

'Position', [X0+(DX*0)+0.78 Y0-(DY*(numy-0.8)) X1 Y1],'FontSize',FSize, 'Visible', 'off');

F_kCl=uicontrol (TABS (2), 'Style', 'edit', 'String', 1, 'units', 'normalized',...

'Position', [X0+(DX*0)+0.78 Y0-(DY*numy) X1 Y1],'FontSize',FSize,'Visible', 'off');

numy=numy+2;

uicontrol (TABS (2), 'Style', 'text', 'String', 'Capsule 2', 'units', 'normalized',...

'Position', [X0+(DX*numx) Y0-(DY*numy) X1 Y1],'FontWeight','bold', 'FontSize',FSize);

$\mathrm{kC2}$ check =

uicontrol (TABS (2), 'Style', 'popupmenu' , 'String', \{'Constant', 'Polynomial', 'Power'\}, 'units', 'normali

zed',...

'Position', [X0+(DX*numx) +0.12 Y0-(DY*numy) X1

Y1], 'FontSize', FSize, 'Callback', (akC2CHECKER) ;

A_kC2_lab=uicontrol (TABS (2), 'Style', 'text', 'String', 'po', 'units', 'normalized',... 'Position', $[\mathrm{X} 0+(\mathrm{DX}$ (0)+0.23 Y0-(DY* (numy-0.8)) X1 Y1], 'FontSize',FSize,'Visible', 'off');

A_kC2_lab2=uicontrol (TABS (2), 'Style', 'text', 'String', 'A', 'units', 'normalized', ...

'Position', $[\mathrm{X} 0+(\mathrm{DX}$ (0) +0.23 Y0-(DY* (numy-0.8)) X1 Y1], 'FontSize',FSize, 'Visible', 'off') ;

A_kC2=uicontrol (TABS (2), 'Style', 'edit', 'String', 1, 'units', 'normalized',...

'Position', [X0+(DX*0)+0.23 Y0-(DY*numy) X1 Y1], 'FontSize',FSize, 'Visible', 'off');

B_kC2_lab=uicontrol(TABS (2), 'Style', 'text', 'String ', 'pl', 'units', 'normalized',...

'Position', [X0+(DX*0)+0.34 Y0-(DY*(numy-0.8)) X1 Y1],'FontSize', FSize, 'Visible', 'off') ;

B kC2 lab2=uicontrol (TABS (2), 'Style', 'text', 'String', 'B', 'units', 'normalized', ...

'Position', [X0+(DX*0)+0.34 Y0-(DY*(numy-0.8)) X1 Y1],'FontSize', FSize, 'Visible', 'off') ;

B kC2=uicontrol (TABS (2), 'Style', 'edit', 'String', 1, 'units', 'normalized',...

'Position', [X0+(DX*0)+0.34 Y0-(DY*numy) X1 Y1],'FontSize',FSize, 'Visible', 'off');

C_kC2_lab=uicontrol(TABS (2), 'Style', 'text', 'String', 'p2', 'units', 'normalized', ...

'Position', [X0+(DX*0)+0.45 Y0-(DY*(numy-0.8)) X1 Y1], 'FontSize', FSize, 'Visible', 'off');

C_kC2_lab2=uicontrol (TABS (2), 'Style', 'text', 'String', 'C', 'units', 'normalized',...

'Position', [X0+(DX*0)+0.45 Y0-(DY*(numy-0.8)) X1 Y1],'FontSize', FSize, 'Visible', 'off') ;

C_kC2=uicontrol (TABS (2), 'style', 'edit', 'String', 1, 'units', 'normalized',...

'Position', [X0+(DX*0)+0.45 Y0-(DY*numy) X1 Y1],'FontSize',FSize, 'Visible','off');

D_kC2_lab=uicontrol (TABS (2), 'Style', 'text', 'String', 'p3', 'units', 'normalized', ...

'Position', [X0+(DX*0)+0.56 Y0-(DY*(numy-0.8)) X1 Y1], 'FontSize',FSize, 'Visible', 'off') ;

D_kC2=uicontrol (TABS (2), 'Style', 'edit', 'String', 1, 'units', 'normalized',...

'Position', [X0+(DX*0)+0.56 Y0-(DY*numy) X1 Y1], 'FontSize', FSize, 'Visible', 'off');

E_kC2_lab=uicontrol (TABS (2), 'Style', 'text', 'String', 'p4', 'units', 'normalized',...

'Position', [X0+(DX*0)+0.67 Y0-(DY* (numy-0.8)) X1 Y1], 'FontSize', FSize, 'Visible', 'off');

E_kC2=uicontrol (TABS (2), 'Style', 'edit', 'String', 1, 'units', 'normalized',...

'Position', [X0+(DX*0)+0.67 Y0-(DY*numy) X1 Y1],'FontSize',FSize,'Visible', 'off');

F_kC2_lab=uicontrol (TABS (2), 'Style', 'text', 'String', 'p5', 'units', 'normalized',...

'Position', [X0+(DX*0)+0.78 Y0-(DY*(numy-0.8)) X1 Y1], 'FontSize', FSize, 'Visible', 'off');

F_kC2=uicontrol (TABS (2), 'Style', 'edit', 'String', 1, 'units', 'normalized',...

'Position', [X0+(DX*0)+0.78 Y0-(DY*numy) X1 Y1],'FontSize',FSize, 'Visible', 'off');

numy=numy+2;

uicontrol (TABS (2), 'Style', 'text', 'String', 'Rodlet', 'units', 'normalized',...

'Position', [X0+(DX*numx) Y0-(DY*numy) X1 Y1], 'FontWeight', 'bold', 'FontSize', FSize);

$\mathrm{kR}$ check =

uic̄ontrol (TABS (2), 'Style', 'popupmenu', 'String', \{'Constant', 'Polynomial', 'Power'\}, 'units', 'normali zed',$\ldots$

'Position', [X0+(DX*numx) +0.12 YO- (DY*numy) X1

Y1], 'FontSize', FSize, 'Callback', (akRCHECKER) ;

A_kR_lab=uicontrol (TABS (2), 'Style', 'text', 'String', 'po', 'units', 'normalized',...

'Position', [X0+(DX*0)+0.23 Y0-(DY*(numy-0.8)) X1 Y1], 'FontSize',FSize, 'Visible', 'off') ;

A kR lab2=uicontrol (TABS (2), 'Style', 'text', 'String', 'A', 'units', 'normalized',...

'Position', [X0+(DX*0)+0.23 Y0-(DY*(numy-0.8)) X1 Y1],'FontSize',FSize, 'Visible','off'); 
A_kR=uicontrol (TABS (2), 'Style', 'edit', 'String', 1, 'units', 'normalized',...

'Position', [X0+(DX*0)+0.23 Y0-(DY*numy) X1 Y1],'FontSize',FSize, 'Visible','off') ;

B kR lab=uicontrol(TABS (2), 'Style', 'text',' 'String', 'pl', 'units', 'normalized',...

'Position', [X0+(DX*0)+0.34 Y0-(DY* (numy-0.8)) X1 Y1],'FontSize',FSize, 'Visible', 'off');

B_kR_lab2=uicontrol (TABS (2), 'Style', 'text',' String', 'B', 'units', 'normalized',...

'Position', [X0+(DX*0)+0.34 Y0-(DY*(numy-0.8)) X1 Y1],'FontSize',FSize, 'Visible','off');

B_kR=uicontrol (TABS (2), 'Style', 'edit', 'String', 1, 'units', 'normalized', ...

'Position', [X0+(DX*0)+0.34 Y0-(DY*numy) X1 Y1],'FontSize',FSize, 'Visible','off');

C_kR_lab=uicontrol (TABS (2), 'Style', 'text', 'String', 'p2', 'units', 'normalized',...

'Position', [X0+(DX*0)+0.45 Y0-(DY*(numy-0.8)) X1 Y1],'FontSize',FSize, 'Visible', 'off'):

C_kR_lab2=uicontrol (TABS (2), 'Style', 'text',' String', 'C', 'units', 'normalized',...

'Position', [X0+(DX*0)+0.45 Y0-(DY*(numy-0.8)) X1 Y1],'FontSize',FSize, 'Visible', 'off');

C_kR=uicontrol (TABS (2), 'Style', 'edit', 'String', 1, 'units', 'normalized',...

'Position', [X0+(DX*0)+0.45 Y0-(DY*numy) X1 Y1],'FontSize',FSize, 'Visible', 'off');

D kR lab=uicontrol (TABS (2),'Style','text',' String', 'p3', 'units', 'normalized',...

'Position', [X0+(DX*0)+0.56 Y0-(DY*(numy-0.8)) X1 Y1],'FontSize', FSize, 'Visible', 'off');

D kR=uicontrol (TABS (2), 'Style', 'edit' ',String', 1, 'units', 'normalized',...

'Position', [X0+(DX*0)+0.56 Y0-(DY*numy) X1 Y1],'FontSize',FSize, 'Visible', 'off');

E kR lab=uicontrol(TABS (2), 'Style', 'text',' String', 'p4', 'units', 'normalized',...

'Position', [X0+(DX*0)+0.67 Y0-(DY*(numy-0.8)) XI Y1],'FontSize',FSize, 'Visible', 'off');

E kR=uicontrol (TABS (2), 'Style', 'edit', 'String', 1, 'units', 'normalized',...

'Position', [X0+(DX*0)+0.67 Y0-(DY*numy) X1 Y1],'FontSize',FSize, 'Visible','off');

F_kR_lab=uicontrol(TABS(2), 'Style', 'text',' String', 'p5', 'units', 'normalized',...

'Position', [X0+(DX*0)+0.78 Y0-(DY* (numy-0.8)) X1 Y1],'FontSize',FSize, 'Visible', 'off');

F_kR=uicontrol(TABS (2),'Style', 'edit', 'String', 1, 'units', 'normalized',...

'Position', [X0+(DX*0)+0.78 Y0-(DY*numy) X1 Y1],'FontSize',FSize, 'Visible','off');

numy=numy +2 ;

uicontrol (TABS (2), 'Style', 'text', 'String', 'Sample', 'units', 'normalized',...

'Position', [X0+(DX*numx) Y0-(DY*numy) X1 Y1], 'FontWeight', 'bold', 'FontSize',FSize).

$\mathrm{kS}$ check $=$

uičcontrol (TABS (2), 'Style', 'popupmenu', 'String', \{'Constant', 'Polynomial', 'Power'\}, 'units', 'normali

zed', ...

'Position', $\left[\mathrm{X} 0+\left(\mathrm{DX} \mathrm{X}^{\star} \mathrm{num}\right)+0.12 \mathrm{Y} 0-\left(\mathrm{DY} \mathrm{N}^{\star}\right.\right.$ umy $) \mathrm{X} 1$

Y1], 'FontSize', FSize, 'Callback', ( $k$ SCHECKER);

A_kS_lab=uicontrol (TABS (2), 'Style', 'text', 'String', 'po' , 'units', 'normalized',...

'Position', [X0+(DX*0)+0.23 Y0-(DY*(numy-0.8)) XI Y1],'FontSize',FSize, 'Visible', 'off');

A_kS_lab2=uicontrol (TABS (2), 'Style', 'text',' String', 'A' ', 'units', 'normalized',...

'Position', [X0+(DX*0)+0.23 Y0-(DY* (numy-0.8)) X1 Y1],'FontSize',FSize, 'Visible', 'off');

A_kS=uicontrol (TABS (2), 'Style', 'edit', 'String', 1, 'units', 'normalized',...

'Position', [X0+(DX*0)+0.23 Y0-(DY*numy) X1 Y1],'FontSize',FSize, 'Visible', 'off');

B_kS_lab=uicontrol (TABS (2), 'Style', 'text', 'String', 'pl', 'units', 'normalized',...

'Position', [X0+(DX*0)+0.34 Y0-(DY*(numy-0.8)) X1 Y1],'FontSize',FSize, 'Visible', 'off');

B_kS_lab2=uicontrol (TABS (2), 'Style', 'text', 'String', 'B', 'units', 'normalized',...

'Position', [XO+(DX*0)+0.34 Y0-(DY*(numy-0.8)) X1 Y1],'FontSize',FSize,'Visible', 'off');

B kS=uicontrol(TABS (2), 'Style',' 'edit','String', 1, 'units','normalized',...

'Position', [X0+(DX*0)+0.34 Y0-(DY*numy) X1 Y1],'FontSize',FSize, 'Visible', 'off');

C kS lab=uicontrol(TABS (2), 'Style', 'text',' 'String', 'p2', 'units', 'normalized',...

'Position', [X0+(DX*0)+0.45 Y0-(DY* (numy-0.8)) X1 Y1],'FontSize',FSize, 'Visible', 'off') ;

C kS lab2=uicontrol (TABS (2), 'Style', 'text', 'String', 'C', 'units', 'normalized',...

'Position', [X0+(DX*0)+0.45 Y0-(DY*(numy-0.8)) X1 Y1],'FontSize', FSize, 'Visible', 'off');

C kS=uicontrol(TABS (2), 'Style', 'edit', 'String', 1, 'units', 'normalized',...

'Position', [X0+(DX*0)+0.45 Y0-(DY*numy) X1 Y1],'FontSize',FSize, 'Visible', 'off');

D kS lab=uicontrol(TABS(2), 'Style', 'text',' String', 'p3', 'units', 'normalized',...

'Position', [X0+(DX*0)+0.56 Y0-(DY* (numy-0.8)) X1 Y1],'FontSize',FSize, 'Visible', 'off');

D_kS=uicontrol (TABS (2), 'Style', 'edit', 'String', 1, 'units', 'normalized',...

'Position', [X0+(DX*0)+0.56 Y0-(DY*numy) X1 Y1],'FontSize',FSize, 'Visible', 'off');

E_kS_lab=uicontrol (TABS (2), 'Style', 'text', 'String', 'p4', 'units', 'normalized',...

'Position', [X0+(DX*0)+0.67 Y0-(DY* (numy-0.8)) XI Y1],'FontSize',FSize, 'Visible', 'off') ;

E_kS=uicontrol(TABS (2), 'Style', 'edit', 'String', 1, 'units', 'normalized', ...

'Position', [X0+(DX*0)+0.67 Y0-(DY*numy) X1 Y1],'FontSize',FSize, 'Visible', 'off');

F_kS_lab=uicontrol (TABS (2), 'Style', 'text', 'String', 'p5', 'units', 'normalized',...

'Position', [X0+(DX*0)+0.78 Y0-(DY*(numy-0.8)) X1 Y1],'FontSize',FSize, 'Visible', 'off');

F_kS=uicontrol(TABS (2),'Style', 'edit', 'String', 1, 'units', 'normalized',...

'Position', [X0+(DX*0)+0.78 Y0-(DY*numy) X1 Y1],'FontSize',FSize, 'Visible','off');

numy=numy +2 ;

uicontrol (TABS (2), 'Style', 'text','String', 'Gas Gap', 'units', 'normalized',...

'Position', [X0+(DX*numx) Y0-(DY*numy) X1 Y1],'FontWeight','bold', 'FontSize',FSize); 
kGap check =

uicontrol (TABS (2), 'Style', 'popupmenu', 'String', \{'Constant', 'Polynomial', 'Power'\}, 'units', 'normali zed',...

'Position', [X0+(DX*numx)+0.12 Y0-(DY*numy) X1

Y1], 'FontSize', FSize, 'Callback', (akGapCHECKER);

A_kGap_lab0=uicontrol (TABS (2), 'Style', 'text', 'String', 'kGap', 'units', 'normalized',... 'Position', [X0+(DX*0)+0.23 Y0-(DY*(numy-0.8)) X1 Y1],'FontSize',FSize, 'Visible', 'on') ;

A kGap lab=uicontrol (TABS (2), 'Style', 'text', 'String', 'po', 'units', 'normalized',... 'Pósition', [X0+(DX*0)+0.23 Y0-(DY*(numy-0.8)) X1 Y1],'FontSize',FSize, 'Visible', 'off') ; A_kGap_lab2=uicontrol (TABS (2), 'Style', 'text', 'String', 'A', 'units', 'normalized',... 'Position', [X0+(DX*0)+0.23 Y0-(DY*(numy-0.8)) X1 Y1],'FontSize',FSize,'Visible', 'off');

A_kGap=uicontrol (TABS (2), 'Style', 'edit', 'String', 0.13, 'units', 'normalized',... 'Position', [X0+(DX*0)+0.23 Y0-(DY*numy) X1 Y1],'FontSize',FSize,'Visible', 'on');

B_kGap_lab=uicontrol(TABS(2), 'Style','text', 'String', 'pl', 'units', 'normalized',... 'Position', [X0+(DX*0)+0.34 Y0-(DY*(numy-0.8)) X1 Y1],'FontSize',FSize,'Visible','off');

B_kGap_lab2=uicontrol (TABS (2),'Style', 'text', 'String', 'B', 'units', 'normalized', ... 'Position', [X0+(DX*0)+0.34 Y0-(DY*(numy-0.8)) X1 Y1],'FontSize',FSize,'Visible','off');

B_kGap=uicontrol (TABS (2), 'Style', 'edit', 'String', 1, 'units', 'normalized',... 'Position', [X0+(DX*0)+0.34 Y0-(DY*numy) X1 Y1],'FontSize',FSize, 'Visible', 'off') ;

C_kGap_lab=uicontrol (TABS (2), 'Style', 'text', 'String', 'p2', 'units', 'normalized',... 'Position', [X0+(DX*0)+0.45 Y0-(DY*(numy-0.8)) X1 Y1],'FontSize',FSize, 'Visible', 'off');

C_kGap_lab2=uicontrol (TABS (2), 'Style', 'text', 'String', 'C', 'units', 'normalized',... 'Position', [X0+(DX*0)+0.45 Y0-(DY*(numy-0.8)) X1 Y1],'FontSize',FSize, 'Visible', 'off');

C_kGap=uicontrol (TABS (2), 'Style', 'edit', 'String', 1, 'units', 'normalized',... 'Position', [X0+(DX*0)+0.45 Y0-(DY*numy) X1 Y1],'FontSize',FSize, 'Visible','off');

D kGap lab=uicontrol(TABS (2), 'Style', 'text', 'String', 'p3','units', 'normalized', ... 'Position', [X0 ( DX*0)+0.56 Y0-(DY* (numy-0.8)) X1 Y1],'FontSize',FSize, 'Visible', 'off'); D kGap=uicontrol (TABS (2), 'Style', 'edit', 'String', 1, 'units', 'normalized',... 'Position', [X0+(DX*0)+0.56 Y0-(DY*numy) X1 Y1],'FontSize',FSize, 'Visible', 'off');

E kGap_lab=uicontrol(TABS(2), 'Style','text', 'String', 'p4', 'units', 'normalized',... 'Position', [X0+(DX*0)+0.67 Y0-(DY*(numy-0.8)) X1 Y1],'FontSize',FSize, 'Visible', 'off'); E_kGap=uicontrol (TABS (2), 'Style', 'edit', 'String', 1, 'units', 'normalized',... 'Position', [X0+(DX*0)+0.67 Y0-(DY*numy) X1 Y1],'FontSize',FSize, 'Visible','off');

F_kGap_lab=uicontrol(TABS (2), 'Style', 'text', 'String', 'p5', 'units', 'normalized', ... 'Position', [X0+(DX*0)+0.78 Y0-(DY*(numy-0.8)) X1 Y1],'FontSize',FSize,'Visible','off');

F_kGap=uicontrol(TABS (2), 'Style', 'edit', 'String', 1, 'units', 'normalized',... 'Position', [X0+(DX*0)+0.78 Y0-(DY*numy) X1 Y1],'FontSize',FSize, 'Visible', 'off');

numy=numy +2 ;

uicontrol (TABS (2), 'Style', 'text', 'String', 'Thermal Bond', 'units', 'normalized',... 'Position', [X0+(DX*numx) Y0-(DY*numy) X1 Y1],'FontWeight','bold', 'FontSize',FSize) ; kBond_check $=$ uicontrol (TABS (2), 'Style', 'popupmenu', 'String', \{'Constant', 'Polynomial', 'Power'\}, 'units', 'normali zed', ...

'Position', $\left[\mathrm{X} 0+\left(\mathrm{DX} \mathrm{X}^{\star} \mathrm{numx}\right)+0.12\right.$ Y0-(DY*numy) $\mathrm{X} 1$

Y1], 'FontSize', FSize, 'Callback', @kBondCHECKER);

A_kBond_labo=uicontrol (TABS (2), 'Style', 'text', 'String', 'kBond', 'units', 'normalized', ... 'Position', [X0+(DX*0)+0.23 Y0-(DY*(numy-0.8)) X1 Y1],'FontSize',FSize, 'Visible', 'on') ;

A_kBond_lab=uicontrol (TABS (2), 'Style', 'text', 'String', 'po', 'units', 'normalized',... 'Pośsition', [X0+(DX*0)+0.23 Y0-(DY*(numy-0.8)) X1 Y1],'FontSize',FSize, 'Visible', 'off');

A_kBond_lab2=uicontrol (TABS (2), 'Style', 'text', 'String', 'A', 'units', 'normalized', ... 'Pos̄ition', [X0+(DX*0)+0.23 Y0-(DY*(numy-0.8)) X1 Y1],'FontSize',FSize, 'Visible', 'off');

A_kBond=uicontrol (TABS (2), 'Style', 'edit', 'String', 60, 'units', 'normalized',... 'Position', [X0+(DX*0)+0.23 Y0-(DY*numy) X1 Y1],'FontSize',FSize, 'Visible', 'on') ;

B kBond lab=uicontrol(TABS (2), 'Style', 'text', 'String', 'pl', 'units', 'normalized', ... 'Position', [X0+(DX*0)+0.34 Y0-(DY*(numy-0.8)) X1 Y1],'FontSize',FSize,'Visible', 'off');

B kBond lab2=uicontrol (TABS (2), 'Style', 'text', 'String', 'B', 'units', 'normalized', ... 'Position', [X0+(DX*0)+0.34 Y0-(DY*(numy-0.8)) X1 Y1],'FontSize',FSize, 'Visible', 'off');

B kBond=uicontrol(TABS (2), 'Style', 'edit', 'String', 1, 'units', 'normalized',... 'Position', [XO+(DX*0)+0.34 Y0-(DY*numy) XI Y1],'FontSize',FSize, 'Visible', 'off');

C kBond lab=uicontrol (TABS (2), 'Style', 'text', 'String', 'p2', 'units', 'normalized', ... 'Position', [X0+(DX*0)+0.45 Y0-(DY*(numy-0.8)) X1 Y1],'FontSize',FSize,'Visible', 'off');

C_kBond_lab2=uicontrol (TABS (2), 'Style', 'text', 'String', 'C' ', 'units', 'normalized',... 'Position', [X0+(DX*0)+0.45 Y0-(DY*(numy-0.8)) X1 Y1],'FontSize',FSize,'Visible', 'off');

C_kBond=uicontrol(TABS (2), 'Style', 'edit', 'String', 1, 'units', 'normalized',... 'Position', [X0+(DX*0)+0.45 Y0-(DY*numy) X1 Y1],'FontSize',FSize, 'Visible','off');

D_kBond_lab=uicontrol (TABS (2), 'Style', 'text', 'String', 'p3', 'units', 'normalized',... 'Position', [X0+(DX*0)+0.56 Y0-(DY*(numy-0.8)) X1 Y1],'FontSize',FSize, 'Visible', 'off');

D_kBond=uicontrol (TABS (2), 'Style', 'edit', 'String', 1, 'units', 'normalized',... 'Position', [X0+(DX*0)+0.56 Y0-(DY*numy) X1 Y1],'FontSize',FSize, 'Visible', 'off');

E_kBond_lab=uicontrol (TABS (2), 'Style', 'text', 'String', 'p4', 'units', 'normalized',... 


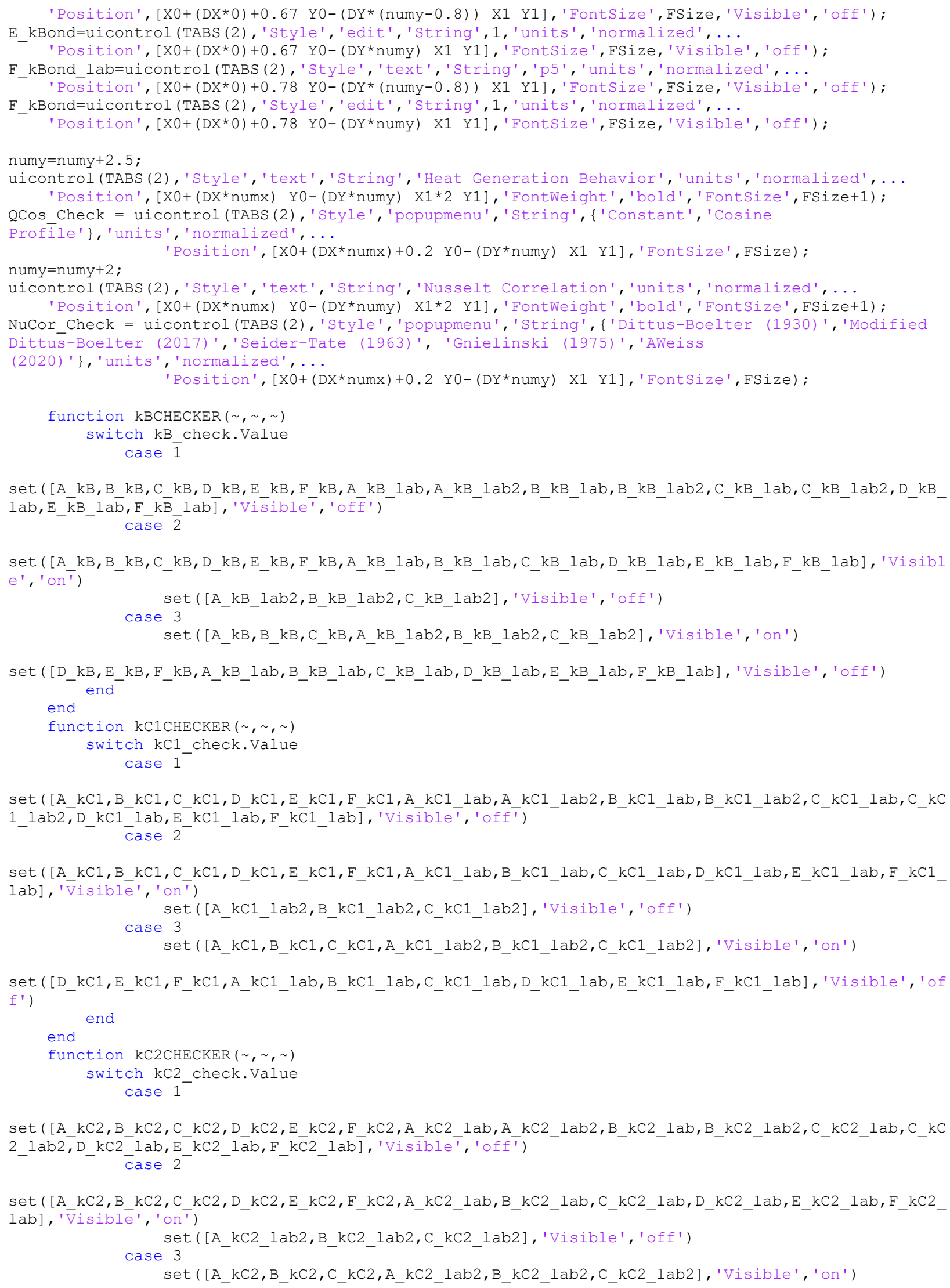




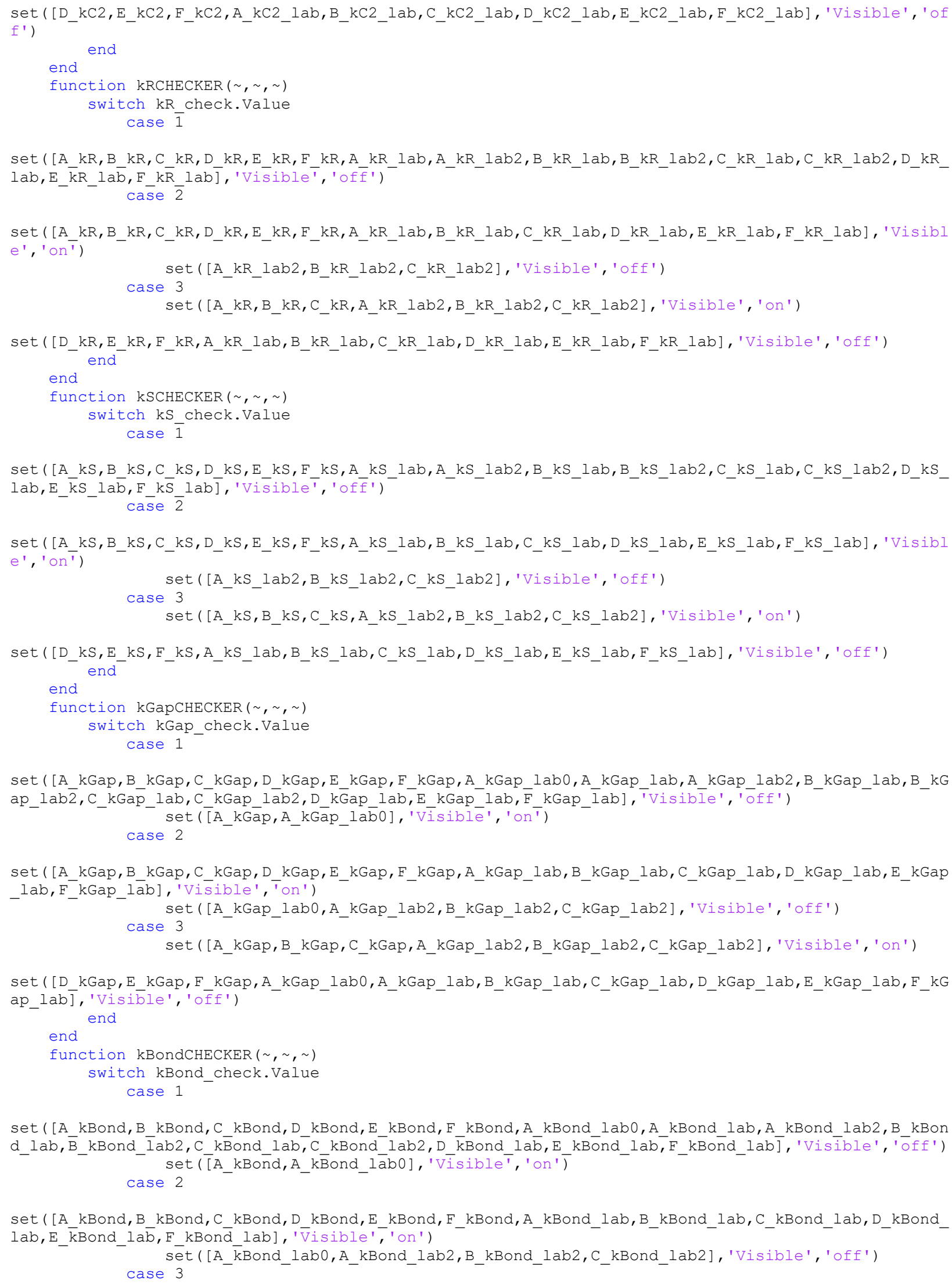


set ([A_kBond, B_kBond, C_kBond, A_kBond_lab2,B_kBond_lab2,C_kBond_lab2], 'Visible', ' on ')

set ( [D_kBond, E_kBond, F_kBond, A_kBond_labo, A_kBond_lab, B_kBond_lab, C_kBond_lab, D_kBond_lab, E_kBond _lab, F_kBond_läb], 'Visíble', 'off' ')

end

end 


\subsection{Go.m}

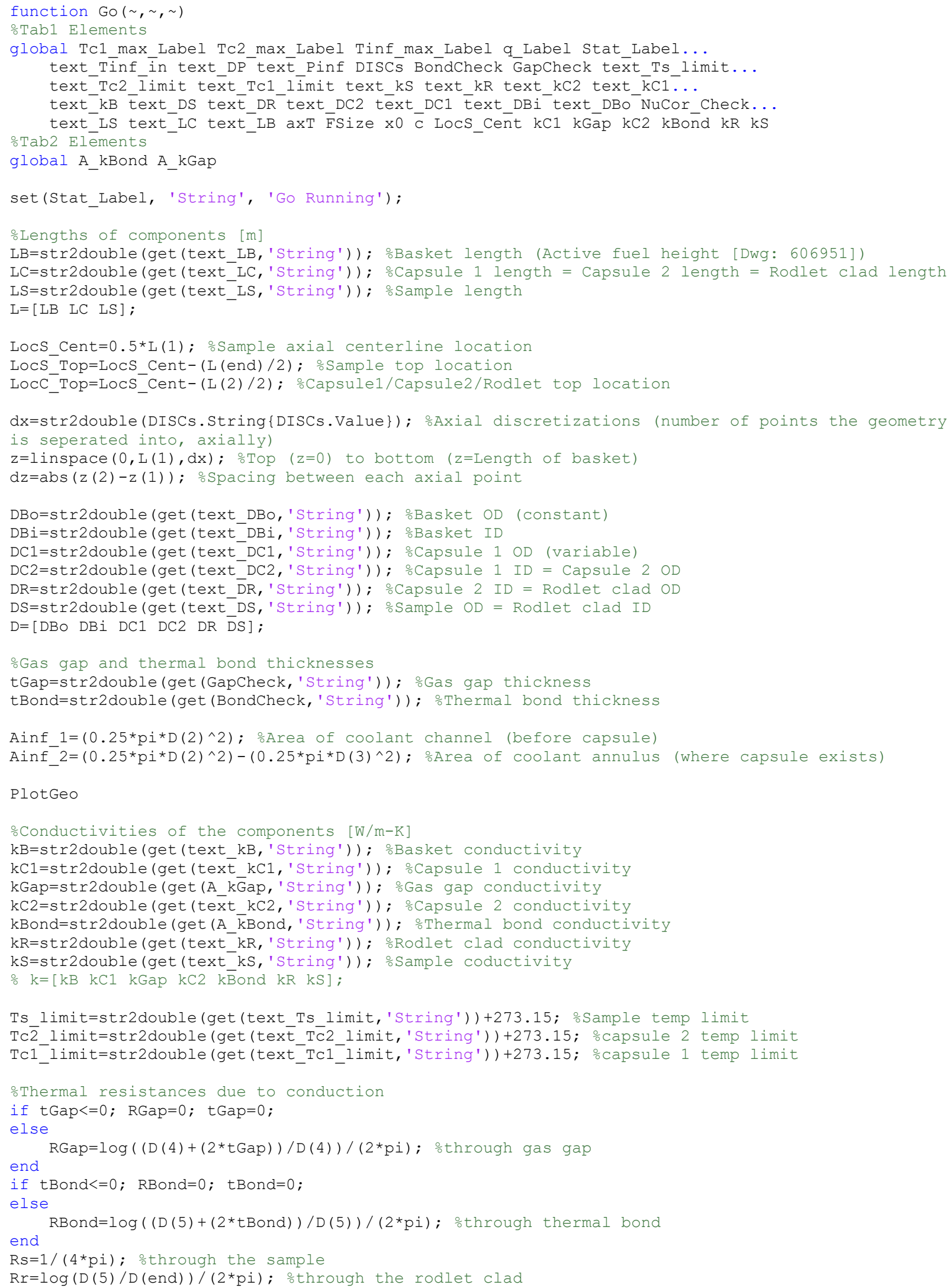


$\mathrm{RC} 2=\log (\mathrm{D}(4) /(\mathrm{D}(5)+(2 *$ tBond $))) /(2 * \mathrm{pi}) ;$ othrough capsule 2

$\operatorname{RCl}=\log (\mathrm{D}(3) /(\mathrm{D}(4)+(2 * \operatorname{tGap}))) /(2 * \mathrm{pi}) ; \%$ through capsule 1

: $\operatorname{Rr}=\log (\mathrm{D}(5) /(\mathrm{D}($ end $)+(2 * 0.0001))) /\left(2 * \mathrm{pi}{ }^{\star} \mathrm{L}(\right.$ end $\left.)\right)$; \%through the rodlet $\mathrm{Clad}$

Rxtra $=\log ((\mathrm{D}($ end $)+(2 * 0.0001)) / \mathrm{D}($ end $)) /(2 * \mathrm{pi}$ L $($ end $))$; \%through a spacer

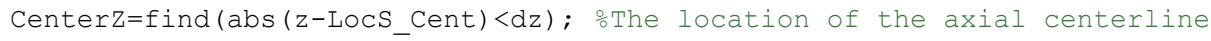

oTemperature of the water coolant in the annulus, based on in and out temps

Tinf in=str2double(get(text Tinf in, 'String')) +273.15; \%Inlet water temp (from the top)

Tinf_out $=70+273.15$; outlet water temp (at the bottom)

\% - Tinf=linspace(Tinf in, Tinf out, dx); \%Linear distribution of the axial water temp

Tinf=ones $(\mathrm{dx}, 1) \cdot{ }^{*} 273.15 ; \operatorname{Tinf}(1)=\operatorname{Tinf}$ in;

Pinf=str2double (get (text Pinf,'String')); \%Pressure of the water [MPa]

$\mathrm{DP}=$ str2double (get (text_D $\overline{\mathrm{P}}$, 'String'))*1E6; $\%$ Pressure Drop [Pa]

$\mathrm{KC}=0.5 ; \mathrm{Ke}=1 ; \operatorname{Re} \mathrm{Kf}=4 \mathrm{E} \overline{6} ;$

$\mathrm{f}=\left(-4 .{ }^{\star} \log \left(\left(0.27 .{ }^{*} 0.26 \mathrm{E}-3 . / \mathrm{D}(2)\right)+\left(7 . / \mathrm{Re} \_\mathrm{Kf}\right) \cdot{ }^{\wedge} 0.9\right)\right) \cdot{ }^{\wedge}-2$;

$\mathrm{Kf}=4 * \mathrm{f} * \mathrm{~L}(1) / \mathrm{D}(2)$;

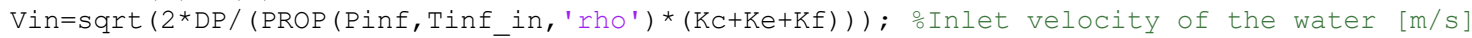

\% $\mathrm{P}=16.5 \mathrm{E} 3 ; \mathrm{C}=0.058 / 0.0254 ; \mathrm{x} 0=-0.6 * 0.0254 ; \% \mathrm{From}$ IN-1260, Table VIII (pg 137 in THAT)

$\mathrm{C}=1 ; \mathrm{x} 0=0 ;$ oCustom constants for a cosine-shaped heat profile of the sample

onitializing temperatures of the components [K]

Ts=ones (length (Tinf), 1) .*273.15; $\div$ Sample temp

Tr=ones (length $($ Tinf), 1$) * * 273.15 ;$ Rodlet clad temp

Tc2=ones (length (Tinf), 1) .*273.15; \%Capsule 2 temp

Tc1=ones $($ length $($ Tinf $), 1) * 273.15 ;$ Capsule 1 temp

Tinf max=Tinf out; Initial Guess

while $\operatorname{ebs}(\max \overline{(T i n f)}$-Tinf $\max )>0.1$

Tinf $\max =\max (\mathrm{Tinf})$;

$\mathrm{M}=\mathrm{PROP}\left(\mathrm{Pinf}, \mathrm{Tinf} \max ,{ }^{\prime} r h 0^{\prime}\right) * \operatorname{Vin} * \operatorname{Ainf} 2$;

for $i=2: \max ($ Centērz)

ological check

Checkl=(z(i) $>=$ LocC Top \&\& z(i) LLocS Top); \%checks if we reached the capsule

Check $2=\left(z(i)>=\right.$ LocS $^{-}$Top $)$; \%checks if we reached the sample

Check=num2str $([$ Check1 Check2 $])$;

switch Check

case ' 10 '

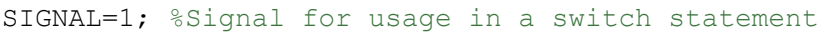

vinf $=M /($ PROP (Pinf, Tinf (i-1), 'rho')*Ainf 2) ;

Dhyd=D (2) $-\mathrm{D}(3)$;

$\mathrm{Deq}=\mathrm{D}(2)-((\mathrm{D}(3) * \mathrm{~L}(2)) / \mathrm{L}(1)) ; \quad$ Equivalent diameter of annulus

case '0 1'

SIGNAL=2; ․osignal for usage in a switch statement

vinf $=\mathrm{M} /(\operatorname{PROP}(\operatorname{Pinf}, \operatorname{Tinf}(i-1)$, 'rho')*Ainf 2);

Dhyd $=\mathrm{D}(2)-\mathrm{D}(3)$;

Deq=D (2) $-((\mathrm{D}(3) * \mathrm{~L}(2)) / \mathrm{L}(1)) ;$ Eqquivalent diameter of annulus

otherwise

SIGNAL $=0$;

vinf $=M /($ PROP (Pinf,Tinf (i-1), 'rho') *Ainf_1) ;

end

[Dhyd, Deq] =deal (D (2));

Re=PROP (Pinf, Tinf (i-1), 'rho') *vinf*Dhyd/PROP (Pinf,Tinf(i-1), 'mu'); \%Reynolds \# switch NuCor_Check.Value

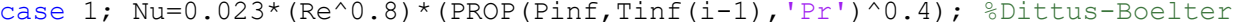
case 2; $\mathrm{Nu}=0.023 *\left(\operatorname{Re}^{\wedge} 0.8\right) *\left(\operatorname{PROP}\left(\operatorname{Pinf}, \operatorname{Tinf}(i-1), \operatorname{Pr}^{\prime}\right) \wedge 0.4\right) *\left(1+\left(0.912^{*}\left(\operatorname{Re}^{\wedge}-\right.\right.\right.$

$\left.\left.0.1) *\left(\operatorname{PROP}\left(\operatorname{Pinf}, \operatorname{Tinf}(i-1), \mathrm{Pr}^{\prime}\right) \wedge 0.4\right) *(1-(2.0043 * \exp (-\operatorname{Dhyd} / \mathrm{D}(3))))\right)\right)$; \%Corrected Dittus-Boelter case 3; $\mathrm{Nu}=0.027 *\left(\operatorname{Re}^{\wedge} 0.8\right)$ * (PROP(Pinf,Tinf (i-1), 'Pr')^(1/3))*((PROP(Pinf,Tinf (i-

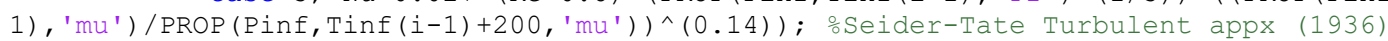

case 4; f_Nu=(0.79*log $(\mathrm{Re})-1.64)^{\wedge}-2 ; \mathrm{Nu}=\left(\left(f \_\mathrm{Nu} / 8\right) *(\mathrm{Re}-1000) * \operatorname{PROP}(\operatorname{Pinf}, \mathrm{Tinf}(i-\right.$

$\left.1),(\operatorname{Pr})) /\left(1+\left(12.7 *\left(\left(\bar{f} \_N u / 8\right) \wedge 0.5\right) *\left(\left(\operatorname{PROP}\left(\operatorname{Pinf}, \operatorname{Tinf}(i-1),{ }^{\prime} \operatorname{Pr}\right)^{\prime}\right)(2 / 3)\right)-1\right)\right)\right)$; $\% \mathrm{Gnielinski}(1975)$

case 5; $\mathrm{Nu}=0.027 *\left(\operatorname{Re}^{\wedge} 0.9\right) *\left(\operatorname{PROP}\left(\operatorname{Pinf}, \mathrm{Tinf}(i-1), \mathrm{Pr}^{\prime}\right) \wedge(0.7)-1\right) *((\operatorname{PROP}(\mathrm{Pinf}, \mathrm{Tinf}(\mathrm{i}-$

1), 'mu')/PROP (Pinf, Tinf (i-1)+200, 'mu'))^(0.14))/abs (1+(20*((f/8)^0.3)*((PROP(Pinf, Tinf (i-

1), 'Pr')^(1/4))))); My custom correlation

end

hinf=Nu*PROP (Pinf,Tinf (i-1), 'k')/Dhyd; oconvection heat transfer coeff.

Rinf=1/(hinf*pi*Deq); othermal resistance due to convection 


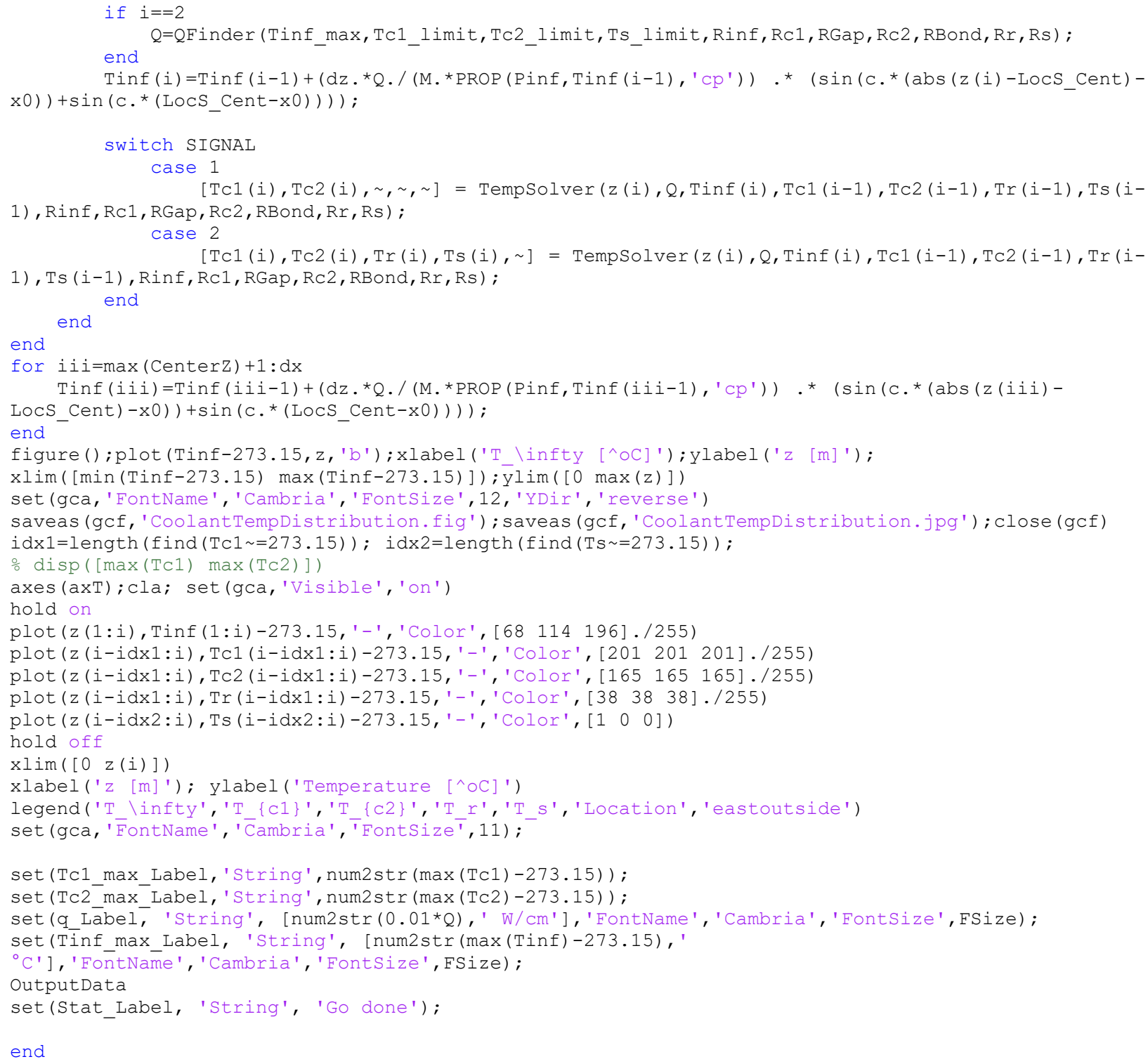




\subsection{Go2.m}

function Go2 ( , , , )

global Tc1_max_Label Tc2_max_Label Tinf_max_Label q_Label Stat_Label...

text_Tinf_in text_DP text_Pinf BondCheck Gapcheck text_Ts_limit...

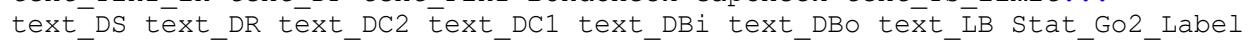

set(Stat_Label, 'String', 'Go2 Running');

op1 $=[$ pwd, ' $\backslash$ Heat_and_Pressure_Limits $\backslash$ ' $]$;

Op2 $=[\mathrm{pwd}$, ' \Coolānt $\overline{\mathrm{M}} \mathrm{ax}$ Temps $\backslash$ '] ;

op3 $=[$ pwd, ' $\backslash$ Samplestats $\backslash ']$;

Op4 $=[$ pwd, ' $\backslash$ tBondstats $\backslash$ ' $]$;

DBi=get (text DBi, 'String'); Basket ID (variable)

DC1=get (text_DC1,'String'); \%Capsule 1 OD (variable)

DC2=get (text_DC2,' String'); \%Capsule 2 OD (variable)

DS=get (text DS, 'String'); \%Sample OD (variable)

tBond=get (BōndCheck, 'String'); othermal bond thickness (variable)

Ts=get (text_Ts_limit,'String'); \%sample temperature limit (variable)

if $\mathrm{DB} i==$ '?'

thik=0.0008; \%Minimum thickness of basket [m]

thikAn=0.0001; \%Minimum thickness of the annulus [m]

$\mathrm{Sy}=170$; $\%$ Yield Strength of Basket [MPa]

$\mathrm{DBo}=$ str2double (get (text_DBo, 'String')) ;

$\mathrm{DC} 1=$ str 2 double $(\mathrm{DC} 1)$;

Frac $=100 *$ round $((\mathrm{DC} 1+(2 * \operatorname{thikAn})) /(\mathrm{DBo}-(2 * \operatorname{thik})), 2)$;

$\mathrm{ct}=0$;

for $q=$ Frac: 99

set (Stat_Go2_Label, 'String', [num2str(100*ct/(99-Frac)), '\% Done']);

$\mathrm{ct}=\mathrm{ct}+1$;

set(text_DBi,' 'String', $\operatorname{num} 2 \operatorname{str}(q * 0.01 *(D B o-(2 * \operatorname{thik}))))$;

Go

Dint (ct) =str2double (get (text DBi, 'String')) ;

QLim (ct) = cell2mat (textscan (get (q_Label, 'String'), '\%f')) ;

$\operatorname{PLim}(\mathrm{ct})=(\mathrm{Sy} * 0.5 *(\operatorname{DBo}-\operatorname{Dint}(\mathrm{ct}))) /((0.5 * \operatorname{Dint}(\mathrm{ct}))+(0.6 * 0.5 *(\operatorname{DBo}-\operatorname{Dint}(\mathrm{ct})))) ; \% \operatorname{Pressure}$

limit of Basket $[\mathrm{MPa}]$

Tinf_max (ct) =cell2mat (textscan (get (Tinf_max_Label, 'String'), '⿳亠口冋'));

end

figure('Units','pixels', 'Position', [308 102862 570])

axl=axes ('Position', [ $\left.\left.\begin{array}{llll}0.1 & 0.1 & 0.8 & 0.8\end{array}\right]\right) ; a=p l o t(a x 1$, Dint, QLim, 'k') ;

xlabel (axl, 'ID_\{Basket\} [m] '); ylabel (axl, 'q' ' $[\mathrm{W} / \mathrm{cm}]$ ');

$\operatorname{ylim}([\min (\mathrm{QLim}), \max (\mathrm{QLim})]) ; x \lim ([\min (\mathrm{Dint}), \max (\mathrm{Dint})])$

set (axl, 'YColor', 'k', 'XColor', 'k', 'Box', 'off', 'Color', 'w', 'XAxisLocation', 'bottom', 'YAxisLocation ', 'left') ;

ax2=axes ('Position', ax1.Position) ; b=plot (ax2, Dint./0.0254,PLim, '-. k') ;

xlabel (ax2,'ID_\{Basket\} [in]'); ylabel(ax2,'Pressure [MPa]');

ylim([min (PLim), $\max (\mathrm{PLim})]) ; x \lim ([\min (\operatorname{Dint} . / 0.0254), \max (\operatorname{Dint./0.0254)])}$

set (ax2,' 'YColor', 'k', 'XColor', 'k', 'Box', 'off', 'Color', 'none', 'XAxisLocation', 'top', 'YAxisLocation ', 'right')

legend([a b], \{'q'' Limit', 'Pressure Limit'\}, 'Location', 'south')

set ([ax1 ax2],' FontName', 'Cambria', 'FontSize', 12);

saveas(gcf,[Op1, 'Basket_Sens.fig']); saveas(gcf,[Op1,'Basket_Sens.jpg']);

figure('Units','pixels','Position', [308 102862 570])

ax1=axes ('Position', [0.1 0.10 .80 .8$]$ ) ; plot(ax1,Dint,Tinf_max, 'k') ;

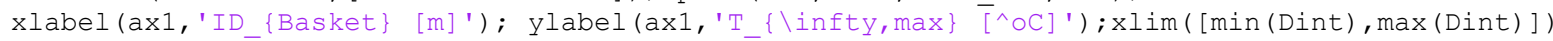

set (axl, 'YColor', 'k', 'XColor', 'k', 'Box', 'off', 'Color', 'w', 'XAxisLocation', 'bottom', 'YAxisLocation ', 'left');

ax2=axes ('Position', ax1.Position); plot(ax2,Dint./0.0254,Tinf_max,'Color', 'none');

xlabel (ax2,' ID_\{Basket\} [in]');xlim([min(Dint./0.0254), max (Diñt./0.0254)])

set (ax2, 'YColor', 'k', 'XColor', 'k', 'Box', 'off', 'Color', 'none', 'XAxisLocation', 'top', 'YAxisLocation ', 'right', 'YTick', []) 
set ([ax1 ax2],'FontName', 'Cambria', 'FontSize', 12);

saveas (gcf, [Op2, 'Basket_Tinf.fig']); saveas(gcf, [Op2, 'Basket_Tinf.jpg']); elseif $\mathrm{DC} 1==$ '?'

thik=0.0008; \%Minimum thickness of capsule 1 [m]

thikAn=0.0001; \%Minimum thickness of annulus [m]

Sy=170; \%Yield Strength of Capsule 1 [MPa]

DBi=str2double (get (text DBi, 'string'));

tGap=str2double (get (GapCheck, 'String')) ;

DC2=str2double (get (text DC2, 'string'));

Frac $=100 *$ round $((\mathrm{DC} 2+(2 *($ tGap+thik $))) /(\operatorname{DBi}-(2 *$ thikAn $)), 2)$;

$\mathrm{ct}=0$;

for $q=$ Frac: 99

set (Stat_Go2_Label, 'String', [num2str(100*ct/(99-Frac)), '\% Done']); $c t=c t+1$

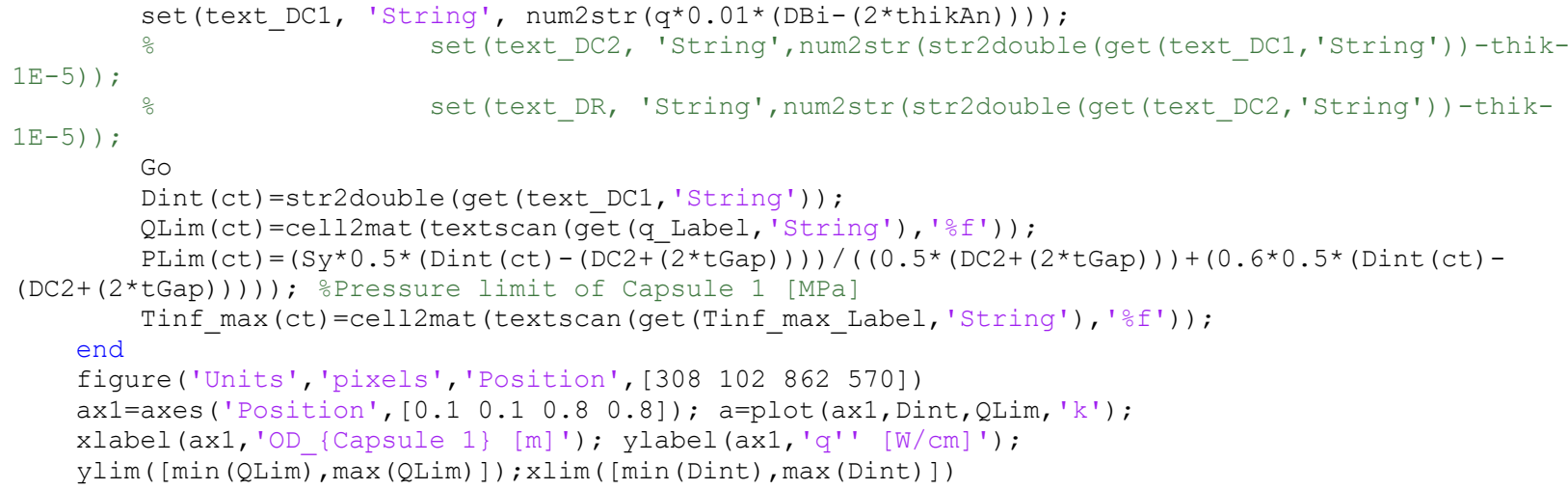




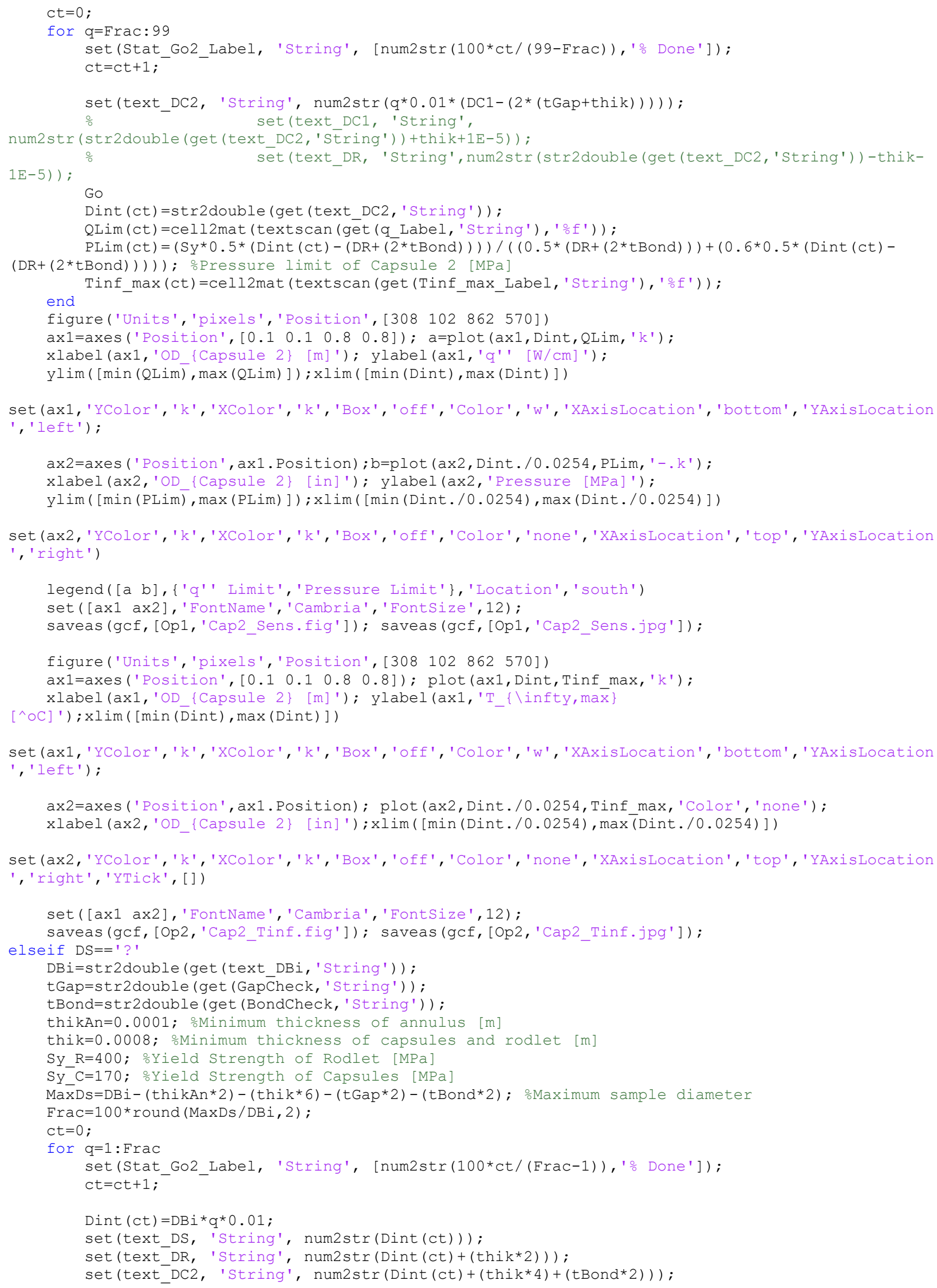


set(text_DC1, 'String', num2str(Dint (ct) $+($ thik*6) $+($ tBond*2) $+($ tGap*2)));

Go

QLim (ct) = cell2mat (textscan (get (q_Label, 'String'), 'ㄷ' ')) ;

Tinf max (ct) =cell2mat (textscan (get (Tinf max Label, 'String'), '⿳f'));

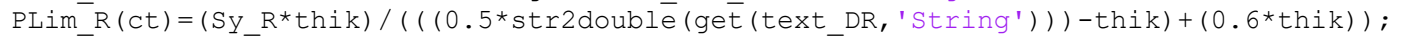

PLim ${ }^{-} 2(\mathrm{ct})=\left(\mathrm{Sy} C^{*}\right.$ thik $) /(((0.5 * \operatorname{str} 2$ double (get (text DC2,'String' $)))-$ thik $\left.)+(0.6 * t h i k)\right)$;

end

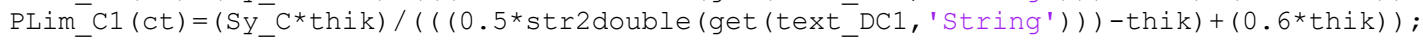

figure('Units', 'pixels','Position', [308 102862 570])

axl=axes ('Position', [0.1 0.10 .80 .8$]$ ); a=plot (ax1, Dint, QLim, 'k') ;

xlabel (axl, 'OD_\{Sample\} [m] '); ylabel (axl, 'q' ' [W/cm] ') ;

$\operatorname{ylim}([\min (\mathrm{QLim}), \max (\mathrm{QLim})]) ; x \lim ([\min (\mathrm{Dint}), \max (\mathrm{Dint})])$

set (axl, 'YColor', 'k', 'XColor', 'k', 'Box', 'off', 'Color', 'w', 'XAxisLocation', 'bottom', 'YAxisLocation ', 'left');

ax2=axes ('Position', ax1.Position); b=plot (ax2, Dint./0.0254, Tinf max,' -.k');

xlabel (ax2,'OD_\{Sample\} [in]'); ylabel(ax2,'T_linfty');

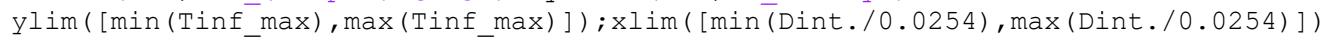

set (ax2,' 'YColor', 'k', 'XColor', 'k', 'Box', 'off', 'Color', 'none', 'XAxisLocation', 'top', 'YAxisLocation ', 'right')

legend([a b], \{'q'' Limit','T \{\infty, max\}'\},'Location', 'best')

set ([ax1 ax2], 'FontName', 'Cambria','FontSize', 12);

saveas(gcf, [Op3, 'Sample Sens.fig']); saveas (gcf, [Op3, 'Sample Sens.jpg']);

figure('Units','pixels','Position', [308 102862 570])

plot (Dint,PLim_R, 'k', Dint,PLim_C2, '--k', Dint,PLim_C1, '-.k')

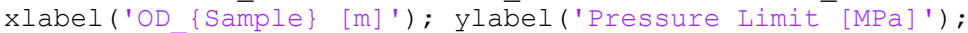

xlim([min(Dint), $\max ($ Dint) ]) ; legend ('Rodlet', 'Capsule 2', 'Capsule 1')

set (gca, 'FontName' , 'Cambria', 'FontSize', 12);

saveas (gcf, [Op3, 'Sample PlimsComps.fig']); saveas (gcf, [Op3, 'Sample PlimsComps.jpg']) ;

elseif tBond=='?'

DBi=str2double (get (text_DBi, 'string')) ;

tGap=str2double (get (Gap Check, 'String')) ;

$\mathrm{DR}=$ str2double (get (text_DR, 'String'));

Sy $\mathrm{C}=170$; \%Yield Strenḡth of Capsules [MPa]

thikAn=0.0002; \%Minimum thickness of annulus [m]

thik=0.0008; Minimum thickness of capsules and rodlet [m]

Min_tBond=1E-5; $\%$ Minimum bond thickness $[\mathrm{m}]$

Max_tBond=(DBi- $(2 * t G a p)-(4 *$ thik $)-(2 *$ thikAn $)-D R) / 2$; $\%$ Max bond thickness

FracH $=$ round (Max tBond/DBi, 2) / (100*Min tBond) ;

FracL=round (Min_tBond/DBi, 2) / (100*Min_tBond);

if $\mathrm{TS}==$ ' ?'

$\mathrm{TS}=500: 100: 2000$;

for $q q=1:$ length (Ts)

set (text Ts limit,' String', num2str(Ts (qq))) ;

$c t=0$;

for $q=$ FracL: $\mathrm{FracH}$

set (Stat Go2 Label, 'String', [num2str(qq), '/', num2str(length(Ts)),

', num2str $\left(100{ }^{*} \mathrm{ct} /((\right.$ FracH-FracL $\left.))\right)$,' 'o Done' ]);

$c t=c t+1$

set (BondCheck, 'String', num2str(DBi*q*Min tBond*100));

tInt (ct, qq) =str2double (get (BondCheck, 'string')) ;

set(text DC1, 'String', num2str (DR+(2*tInt (ct)) +(2*tGap) $+(4 *$ thik)) );

set(text_DC2,' 'String', num $2 \operatorname{str}(\mathrm{DR}+(2 * \operatorname{tn} t(\mathrm{ct}))+(2 *$ thik) $))$;

Go

Tc1 max (ct, qq) =str2double (get (Tc1 max Label, 'String')) ;

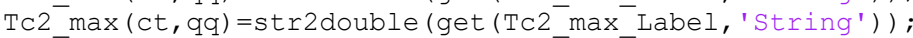

QLim (ct, qq) = cell2mat (textscan (get (q Label, 'String'), '⿳亠䒑 ') ) ;

Tinf_max (ct, qq) =cell2mat (textscan (get (Tinf_max_Label, 'String'), '⿳亠䒑 '));

PLim_C2 $(\mathrm{ct})=\left(\right.$ Sy_C $\left.^{*} \operatorname{thik}\right) /\left(\left(\left(0.5^{*} \operatorname{str} 2 \mathrm{double}(\operatorname{get}(\right.\right.\right.$ text_DC2,'String' $\left.))\right)-$ thik $\left.)+(0.6 * t h i k)\right)$; 


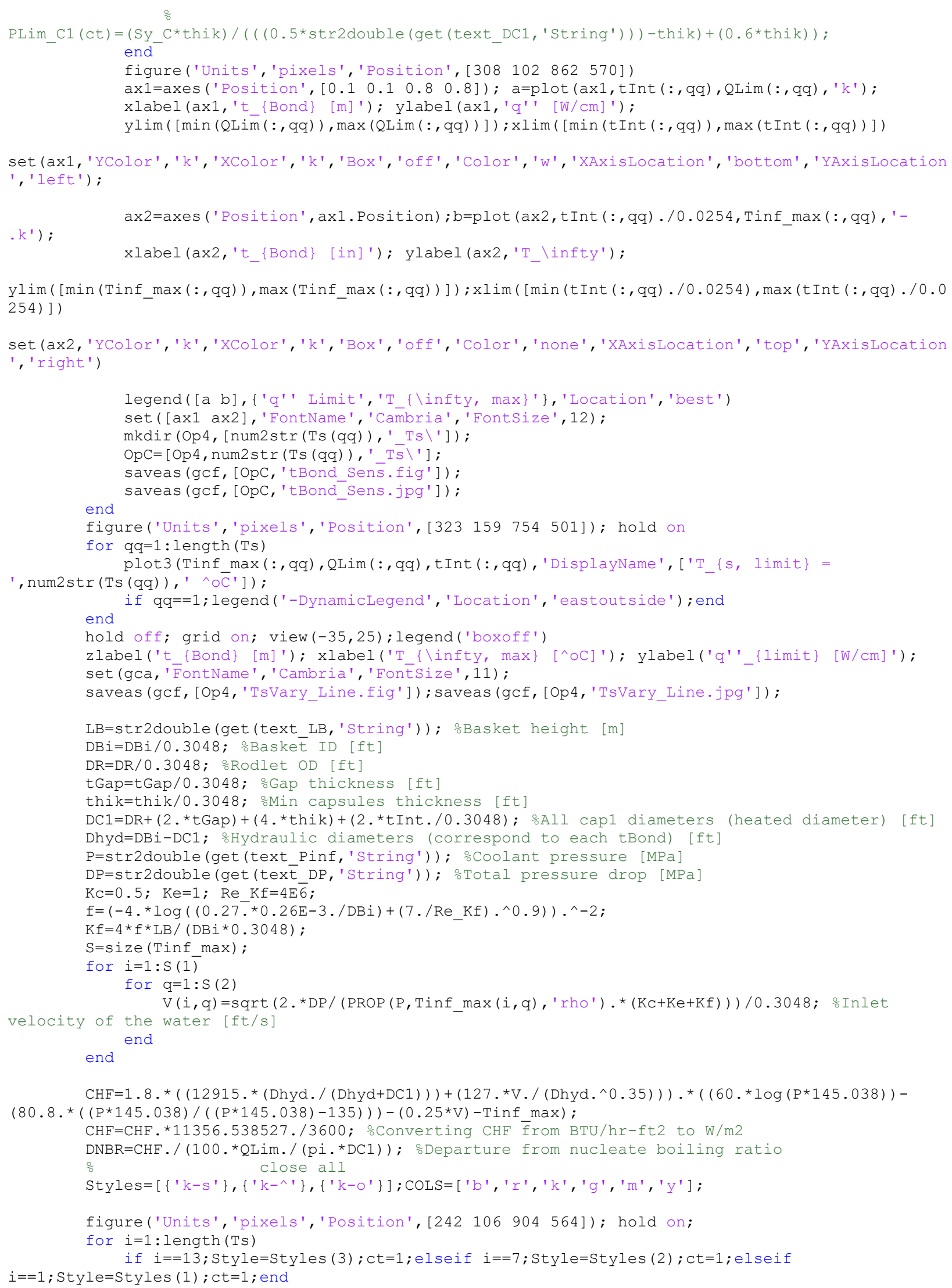




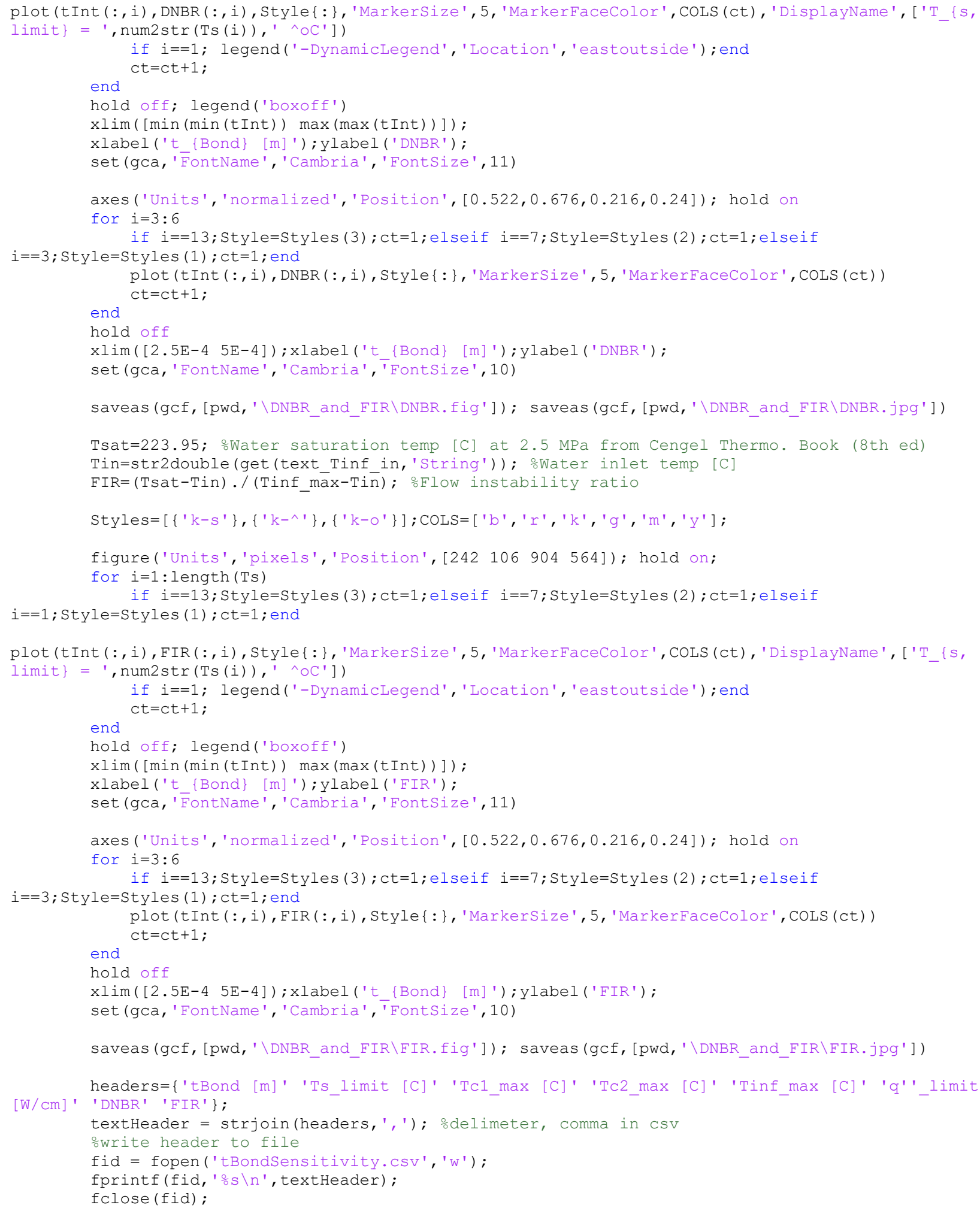

dlmwrite ('tBondSensitivity.CSV', [reshape (tInt', [prod(S),1]), repmat (TS', [S (1), 1]), reshape (Tc1_max' , [prod(S),1]), reshape (TC2 max', [prod(S),1] ), reshape (Tinf max', [prod(S), 1] ), reshape (QLim', [prod (S)

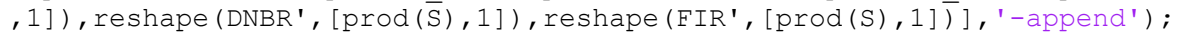




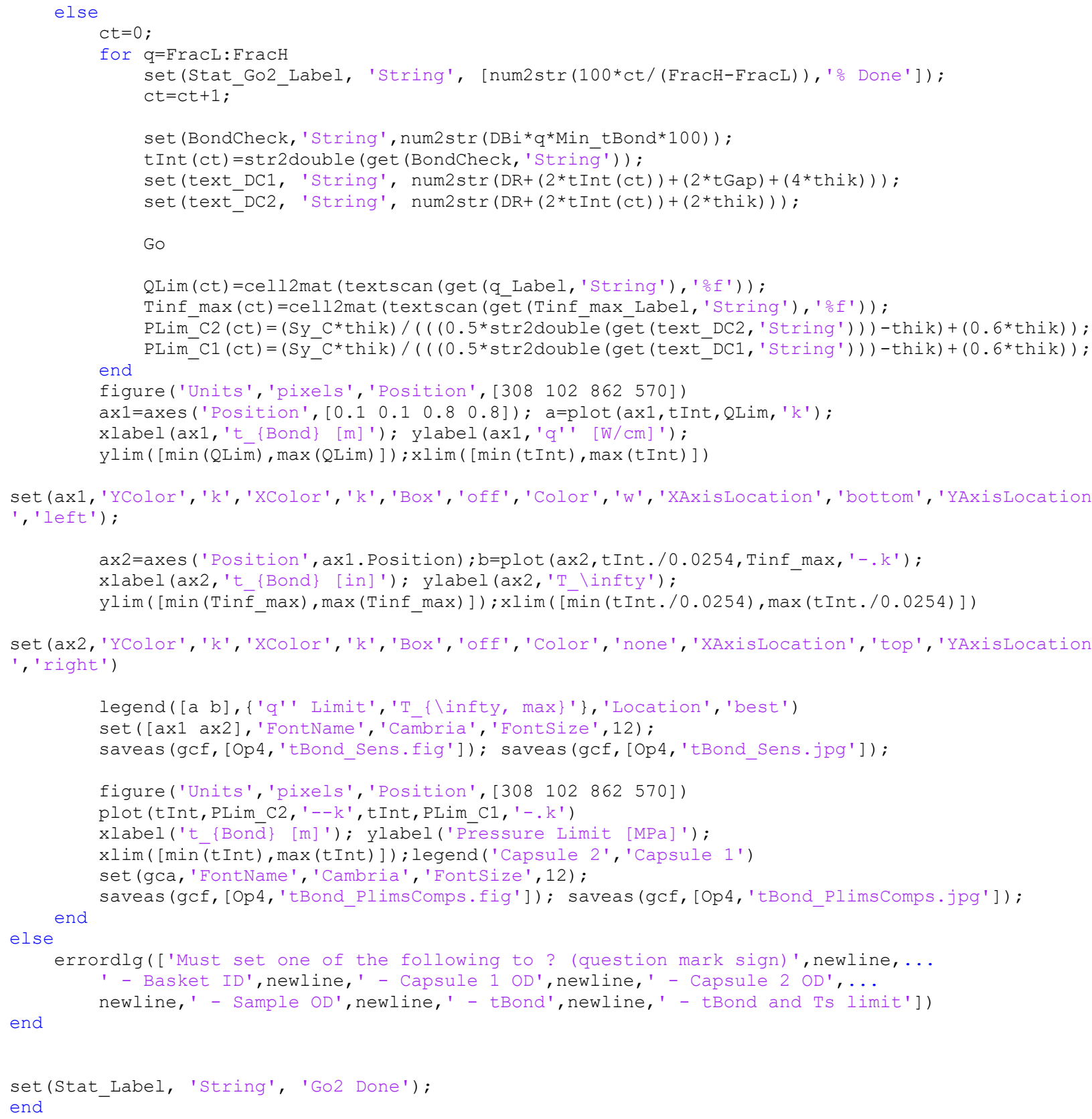




\subsection{Go3.m}

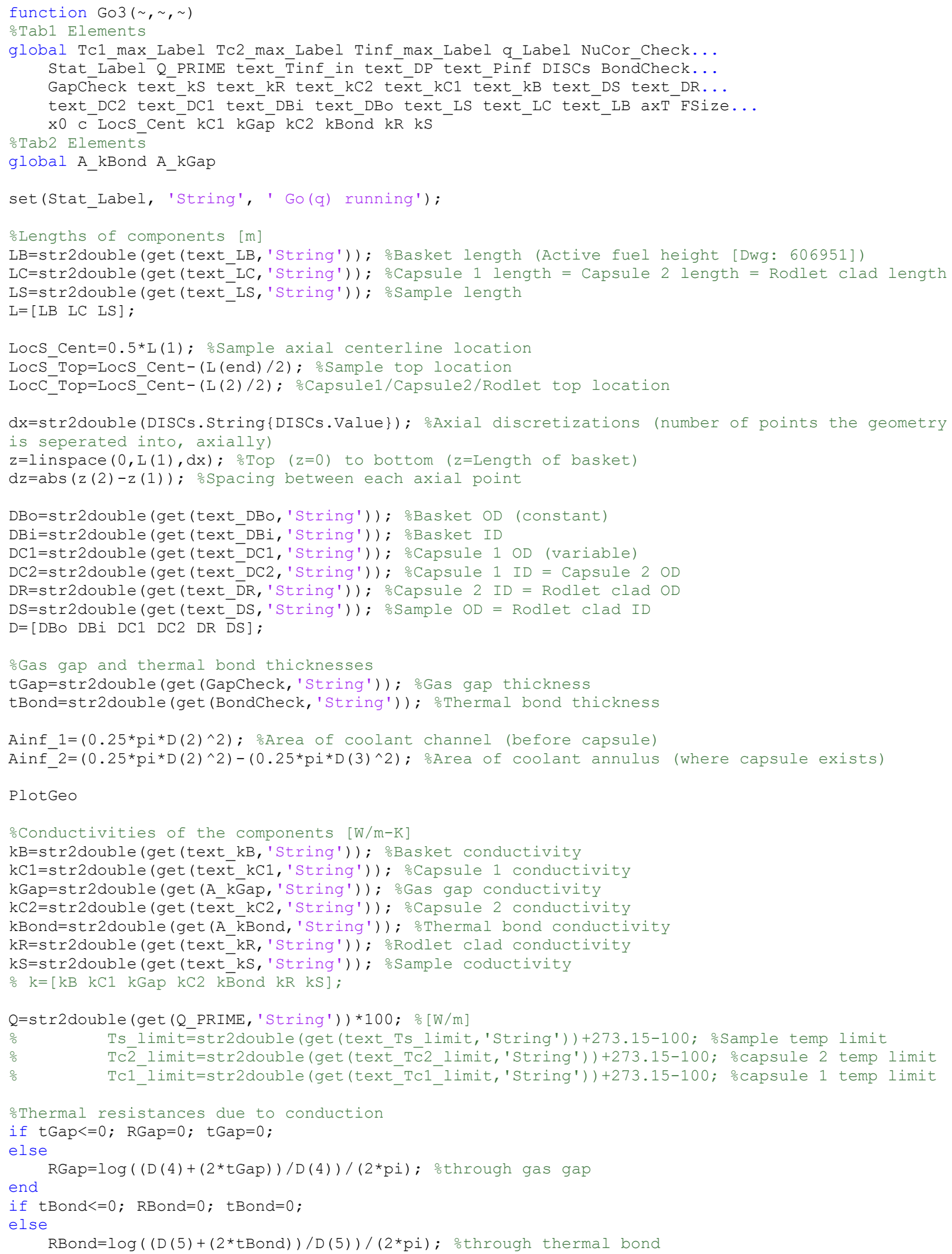


end

$\mathrm{Rs}=1 /(4 * \mathrm{pi})$; othrough the sample

$\mathrm{Rr}=\log (\mathrm{D}(5) / \mathrm{D}($ end $)) /(2 * \mathrm{pi}) ;$ othrough the rodlet clad

$\mathrm{RC} 2=\log (\mathrm{D}(4) /(\mathrm{D}(5)+(2 *$ tBond $))) /(2 * \mathrm{pi}) ;$ \%through capsule 2

$\mathrm{RCl}=\log (\mathrm{D}(3) /(\mathrm{D}(4)+(2 * \operatorname{tGap}))) /(2 * \mathrm{pi}) ;$ othrough capsule 1

\% $\operatorname{Rr}=\log (\mathrm{D}(5) /(\mathrm{D}(\mathrm{end})+(2 * 0.0001))) /\left(2 * \mathrm{pi}{ }^{\star} \mathrm{L}(\mathrm{end})\right)$; \%through the rodlet $\mathrm{Clad}$

Rxtra $=\log ((\mathrm{D}($ end $)+(2 * 0.0001)) / \mathrm{D}($ end $)) /\left(2 * \mathrm{pi}{ }^{\star} \mathrm{L}(\mathrm{end})\right) ;$ \% through a spacer

Centerz=find (abs $\left.\left(z-L o c S \_C e n t\right)<d z\right)$; othe location of the axial centerline

oTemperature of the water coolant in the annulus, based on in and out temps

Tinf_in=str2double (get (text_Tinf_in,'String')) +273.15; \%Inlet water temp (from the top)

Tinf_out $=70+273.15$; outlet water temp (at the bottom)

\% Tinf=linspace(Tinf in, Tinf out, dx); $\frac{\circ}{\circ}$ Linear distribution of the axial water temp Tinf=ones $(d x, 1) . * 273.15 ; \operatorname{Tinf}(1)=\operatorname{Tin} \bar{f}_{-}$in;

Pinf=str2double (get (text_Pinf, 'String')); \%Pressure of the water [MPa]

$\mathrm{DP}=$ str2double (get (text DP, 'String'))*1E6; \%Pressure Drop [Pa]

$\mathrm{KC}=0.5 ; \mathrm{Ke}=1 ; \mathrm{Re} \mathrm{Kf}=4 \mathrm{E} \overline{6}$;

$\mathrm{f}=\left(-4 .{ }^{*} \log \left(\left(0.27 .{ }^{\star} 0.26 \mathrm{E}-3 . / \mathrm{D}(2)\right)+(7 . / \mathrm{Re} \mathrm{Kf}) \cdot{ }^{\wedge} 0.9\right)\right) .^{\wedge}-2$;

$\mathrm{Kf}=4 * \mathrm{f} * \mathrm{~L}(1) / \mathrm{D}(2)$

$\operatorname{Vin}=\operatorname{sqrt}(2 * \mathrm{DP} /(\mathrm{PROP}(\mathrm{Pinf}, \mathrm{Tinf}$ in, 'rho')*(Kc+Ke+Kf)$))$; \%Inlet velocity of the water [m/s]

$\mathrm{P}=\mathrm{Q}^{*} \mathrm{dz}$;

$\mathrm{c}=1 ; \mathrm{x} 0=0 ; \%$ Custom cosine fit for sample power

OInitializing temperatures of the components [K]

Ts=ones (length (Tinf), 1) .*273.15; :Sample temp

Tr=ones (length $($ Tinf) 1 ) $* * 273.15$; Rodlet clad temp

Tc2=ones (length (Tinf), 1) *273.15; \%Capsule 2 temp

TCl=ones (length (Tinf), 1).*273.15; \%Capsule 1 temp

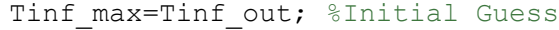

while $\operatorname{ebs}(\max \overline{(T i n f})-T i n f \max )>0.1$

Tinf $\max =\max (\operatorname{Tinf})$;

$\mathrm{M}=\mathrm{PR} \overline{\mathrm{O} P}\left(\mathrm{Pinf}, \mathrm{Tinf} \max\right.$, 'rho' $^{*}{ }^{*} \mathrm{Vin}{ }^{*} \mathrm{Ainf} 2$;

for $i=2: \max ($ Centerz)

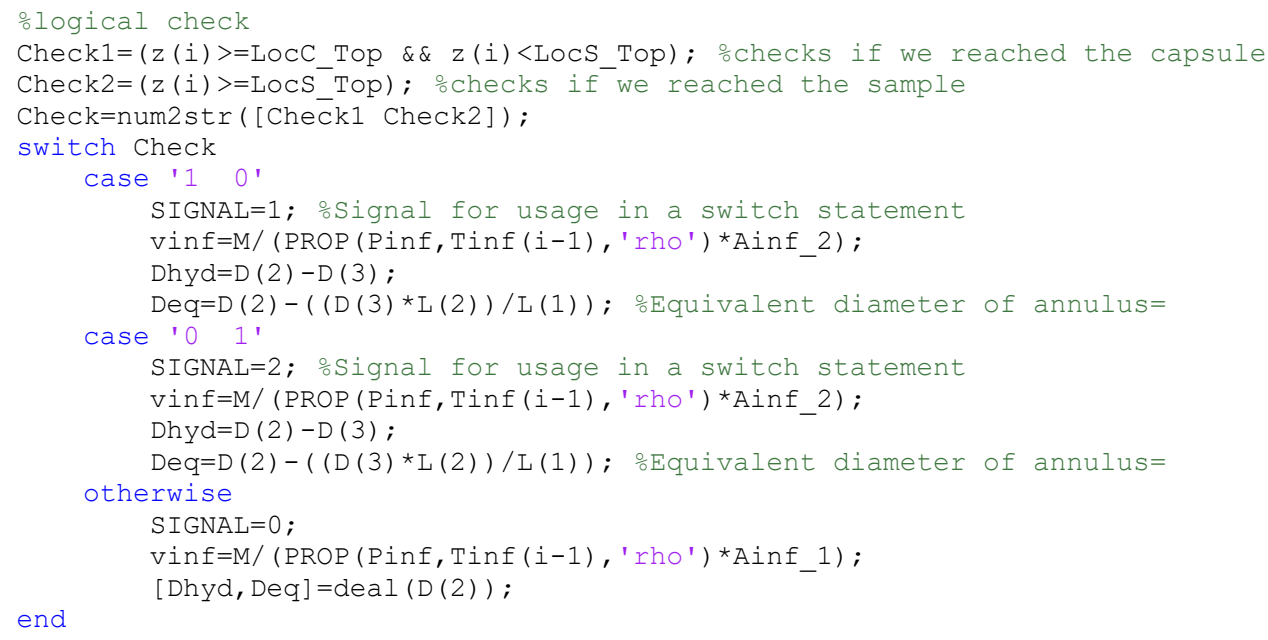




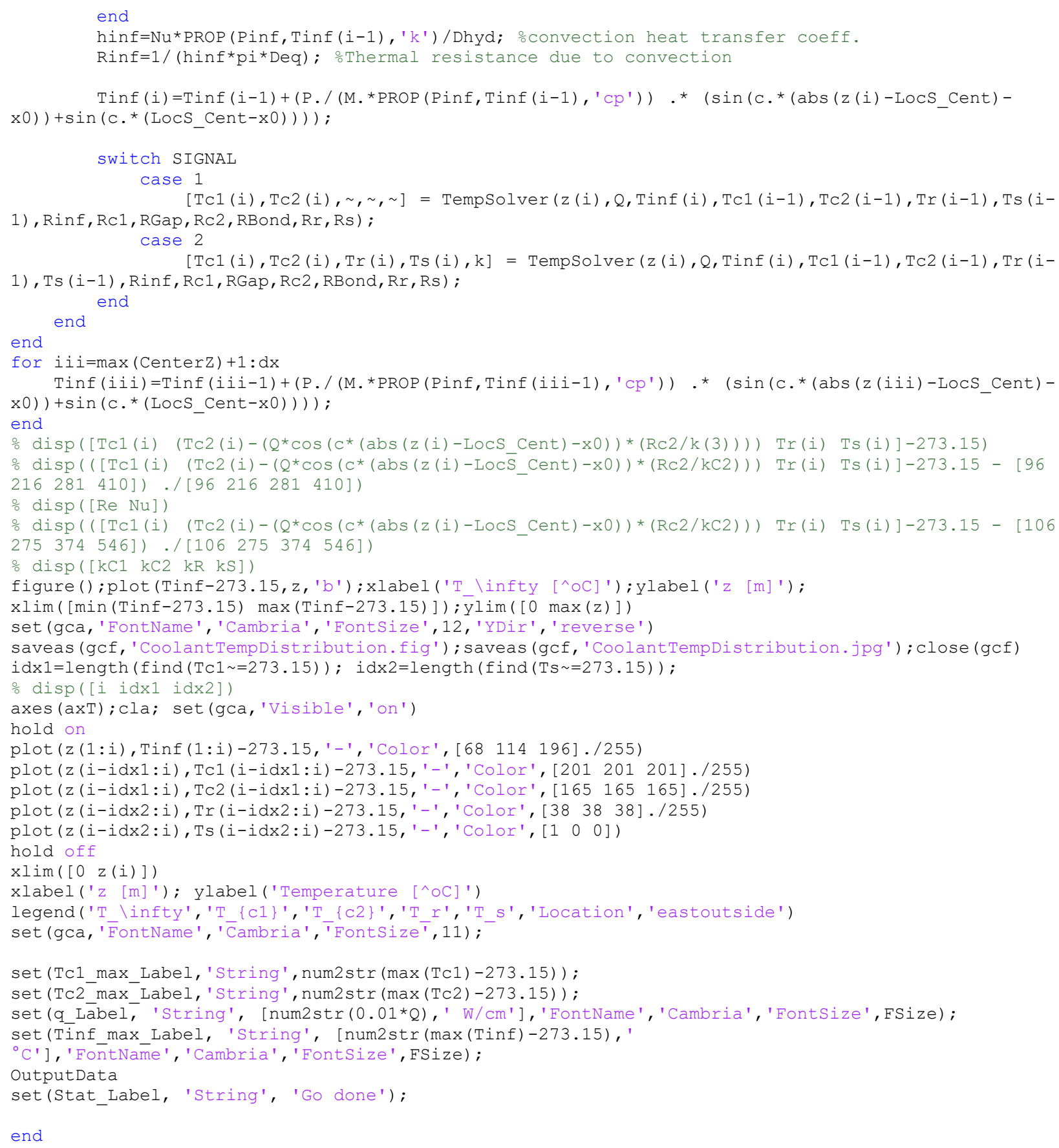




\subsection{PlotGeo.m}

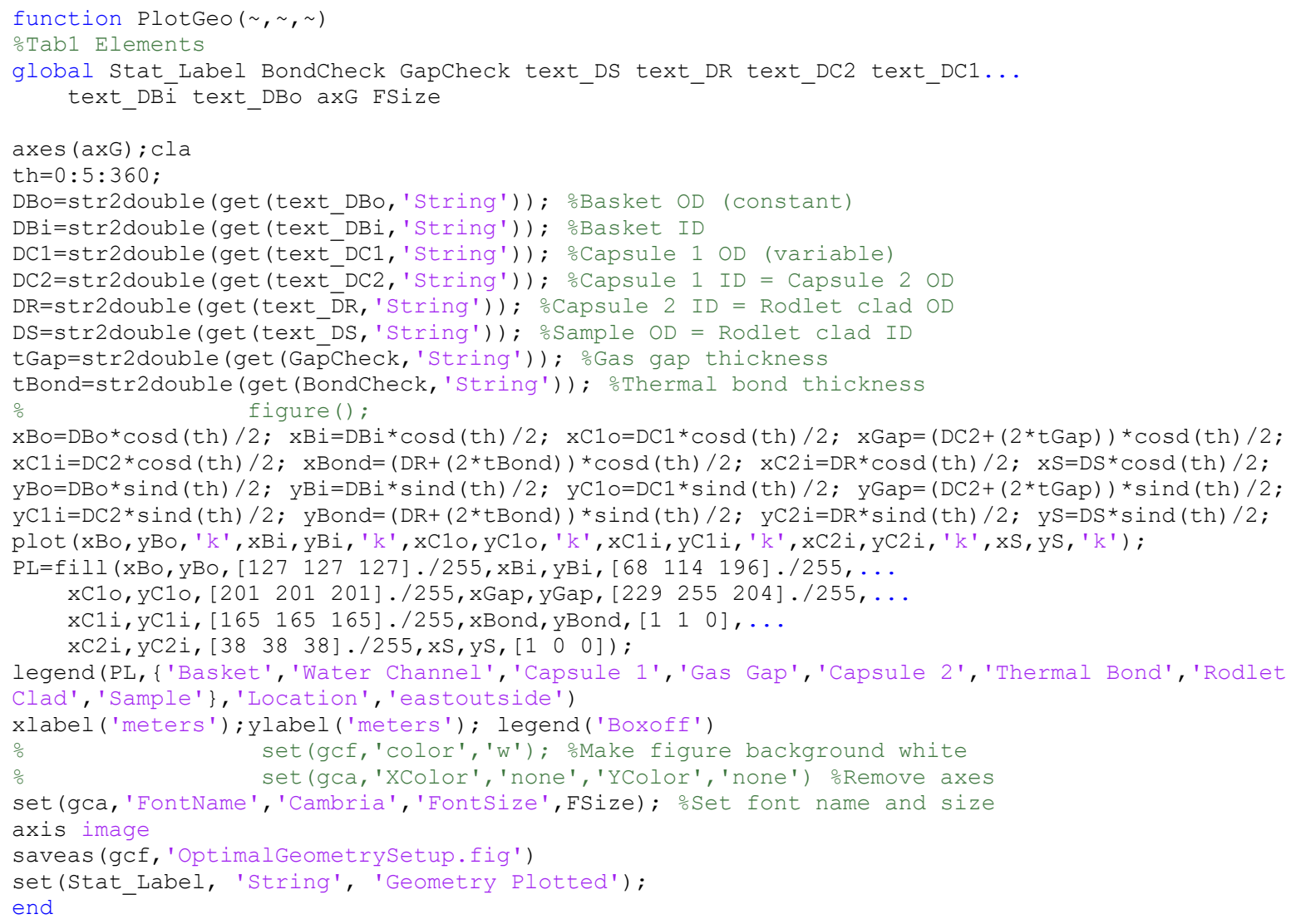




\subsection{OutputData.m}

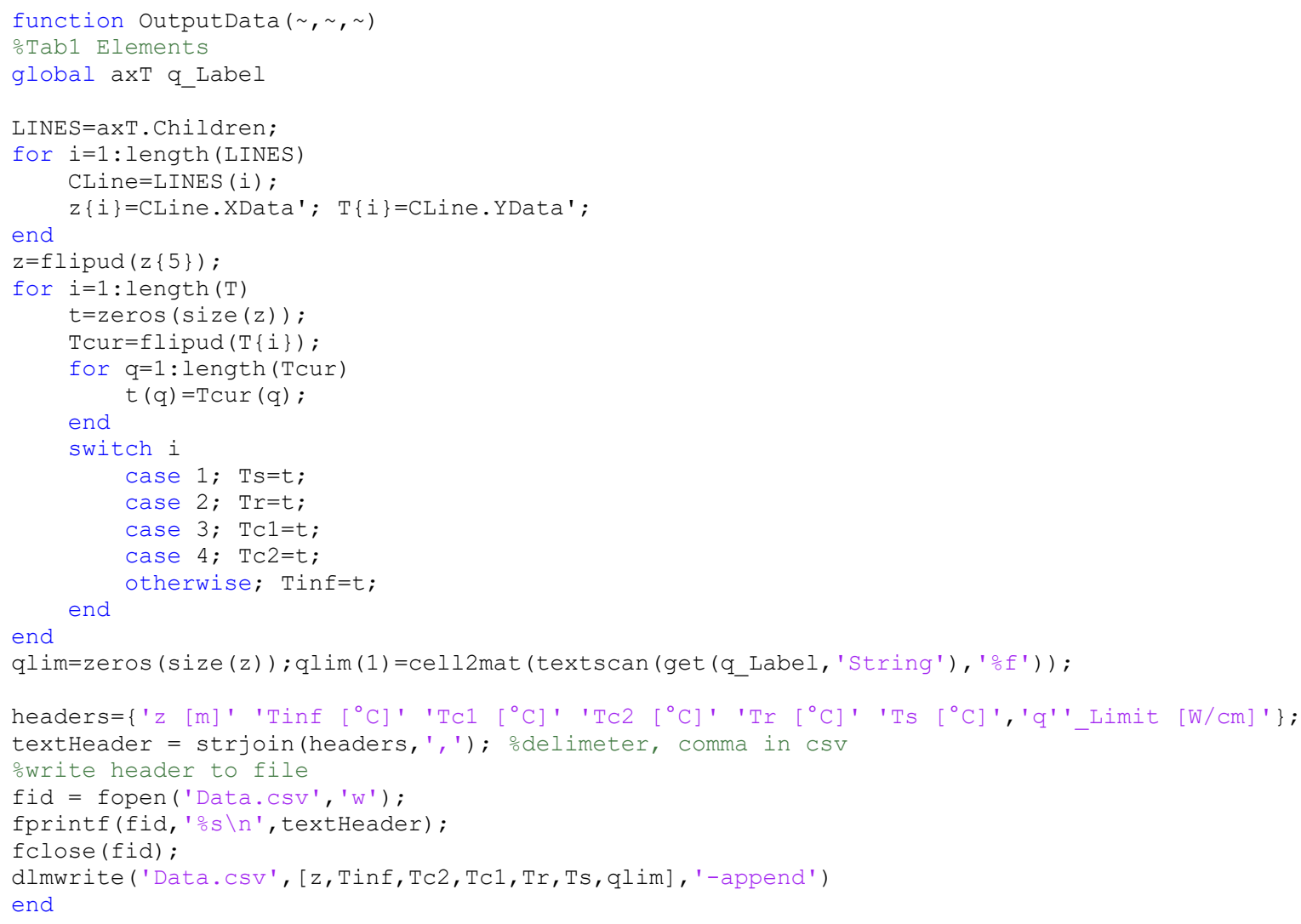




\subsection{TempSolver.m}

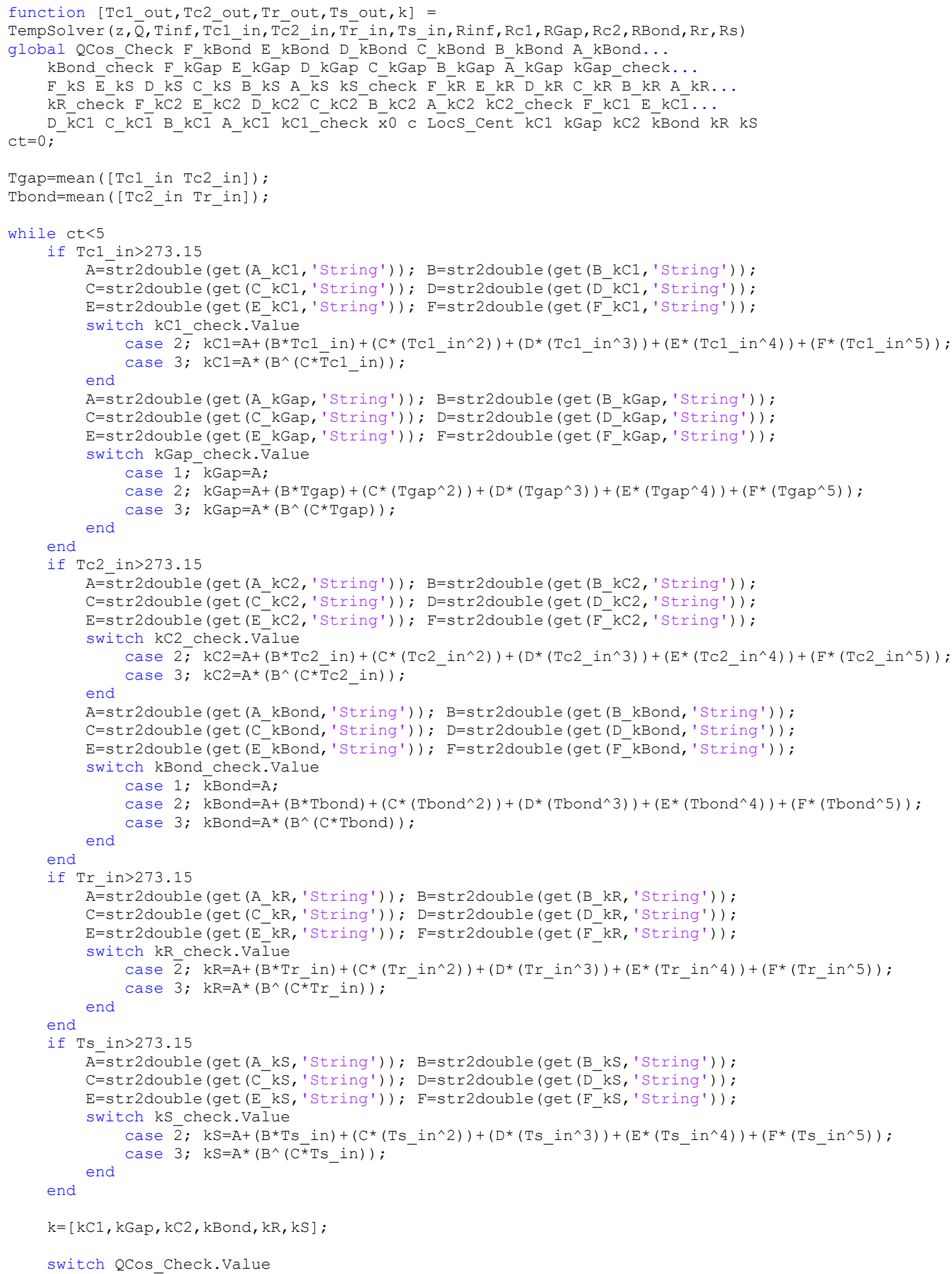




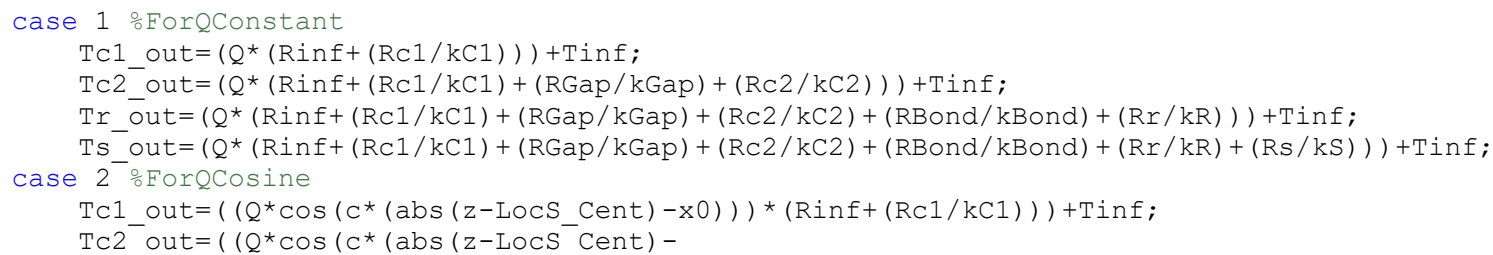




\subsection{QFinder.m}

function $\mathrm{Q}=$ QFinder(Tinf_max, Tc1_limit, Tc2_limit, Ts_limit,Rinf,Rc1, RGap, Rc2,RBond, Rr, Rs)

global $\mathrm{F}$ kBond $\mathrm{E}$ kBond $\mathrm{D} \overline{\mathrm{k} B o n d} \mathrm{C} \overline{\mathrm{k}} \mathrm{B}$ ond $\mathrm{B}$ kBōnd $\mathrm{A}$ kBoñd...

kBond_check F_kGap E_kGap D_k kap C_kGap B_kGap A_kGap kGap_check...

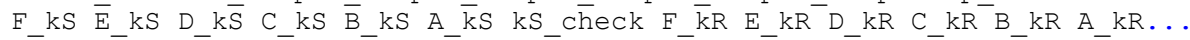

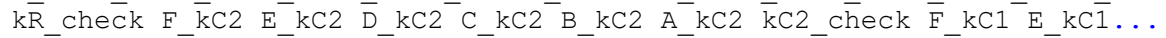

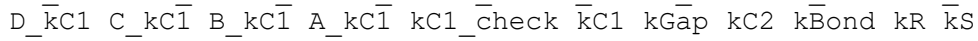

Tc1_in=Tc1_limit;

Tc2 ${ }^{-}$in=Tc2 ${ }^{-}$limit;

Tr_in=mean ([TC2_limit,Ts_limit]);

Ts_in=Ts_limit;

if TC1 in>273.15

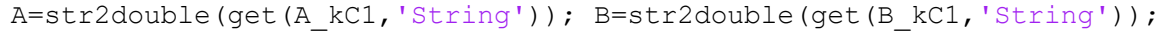

$\mathrm{C}=\operatorname{str} 2$ double (get $\left(\mathrm{C}_{-}^{-} \mathrm{kC1}\right.$, 'String')); $\mathrm{D}=\mathrm{str} 2 \operatorname{double}\left(\operatorname{get}\left(\mathrm{D}_{-}^{-} \mathrm{kC1}\right.\right.$, 'String'));

$\mathrm{E}=\mathrm{str} 2 \mathrm{double}($ get (E kC1, 'String')); $\mathrm{F}=\mathrm{str} 2 \mathrm{double}(\operatorname{get}(\mathrm{F} \mathrm{kC1}$, 'String')) ; switch kC1_check. Välue

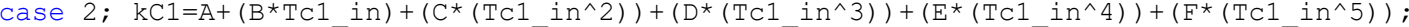

end

case $3 ; \mathrm{kCl}=\mathrm{A}^{*}\left(\mathrm{~B}^{\wedge}\left(\mathrm{C} * \overline{\mathrm{T}} \mathrm{C}{ }_{-}\right.\right.$in $\left.)\right)$;

A=str2double (get (A_kGap, 'String')); B=str2double (get (B_kGap, 'String')) ;

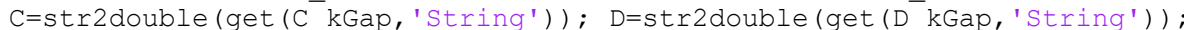

E=str2double (get (E_kGap, 'String')); F=str2double (get (F_kGap, 'String')) ;

switch kGap check. $\bar{V}$ alue

case $1 ; \mathrm{kGap}=\mathrm{A}$;

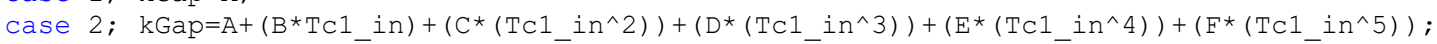

case $3 ; \mathrm{kGap}=\mathrm{A}^{*}\left(\mathrm{~B}^{\wedge}\left(\mathrm{C}^{\star} \mathrm{T} \mathrm{T} 1\right.\right.$ in $\left.)\right)$;

end end

if TC2 in $>273.15$

$\mathrm{A}=\overline{\mathrm{s}} \operatorname{tr} 2$ double (get (A kC2, 'String')); $\mathrm{B}=\mathrm{str} 2 \operatorname{double}(\operatorname{get}(\mathrm{B} \mathrm{kC} 2$, 'String'));

$\mathrm{C}=\operatorname{str} 2$ double (get $\left(\mathrm{C}_{-} \mathrm{kC2}\right.$, 'String')); $\mathrm{D}=\operatorname{str} 2 \operatorname{double}\left(\operatorname{get}\left(\mathrm{D}_{-} \mathrm{kC} 2\right.\right.$, 'String' $\left.)\right)$;

$\mathrm{E}=\operatorname{str} 2$ double (get $\left(\mathrm{E}^{-} \mathrm{kC2}\right.$, 'String')); $\mathrm{F}=\operatorname{str} 2 \operatorname{double}\left(\operatorname{get}\left(\mathrm{F}^{-} \mathrm{kC2}\right.\right.$, 'String'));

switch kC2 check. Value

case $2 \bar{i} \mathrm{kC} 2=\mathrm{A}+\left(\mathrm{B}^{\star} \mathrm{TC} 2 \mathrm{in}\right)+\left(\mathrm{C}^{\star}\left(\mathrm{TC} 2 \mathrm{In}^{\wedge} 2\right)\right)+\left(\mathrm{D}^{\star}\left(\mathrm{TC} 2 i \mathrm{n}^{\wedge} 3\right)\right)+\left(\mathrm{E}^{\star}\left(\mathrm{TC} 2 \mathrm{in}^{\wedge} 4\right)\right)+\left(\mathrm{F}^{\star}\left(\mathrm{TC} 2 \mathrm{in}^{\wedge} 5\right)\right) ;$

end case $3 ; \mathrm{kC} 2=\mathrm{A}^{\star}\left(\mathrm{B}^{\wedge}\left(\mathrm{C}^{\star} \mathrm{TC} 2 \mathrm{in}\right)\right)$;

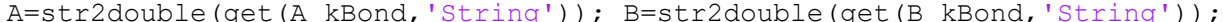

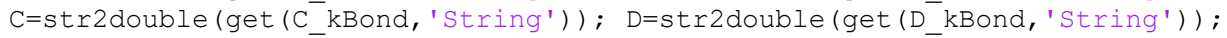

$\mathrm{E}=\mathrm{str} 2$ double (get $\left(\mathrm{E}^{-} \mathrm{kBond}\right.$, 'String')); $\mathrm{F}=\mathrm{str} 2 \mathrm{double}\left(\operatorname{get}\left(\mathrm{F}^{-} \mathrm{kBond}\right.\right.$, 'String')) ;

switch kBond_check. Value

case $1 ; \overline{\mathrm{k} B}$ ond $=\mathrm{A}$;

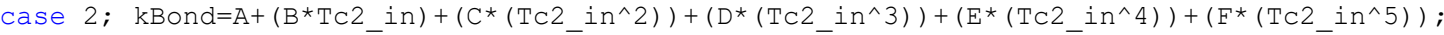
end

case $3 ; \mathrm{kBond}=\mathrm{A}^{*}\left(\mathrm{~B}^{\wedge}(\mathrm{C} * \overline{\mathrm{T}} \mathrm{C} 2\right.$ in $\left.)\right)$

end

if $\operatorname{Tr}$ in $>273.15$

$\mathrm{A}=\operatorname{str} 2$ double (get (A_kR, 'String')); $B=\operatorname{str} 2 \operatorname{double}(\operatorname{get}(\mathrm{B}$ kR, 'String'));

$\mathrm{C}=$ str2double (get ( $\mathrm{C}^{-} \mathrm{kR}$, 'String')); $\mathrm{D}=$ str2double (get ( ${ }^{-} \mathrm{kR}$, 'String'));

$\mathrm{E}=\operatorname{str} 2$ double $\left(\operatorname{get}\left(\mathrm{E}_{-}^{-} \mathrm{kR}\right.\right.$, 'String')); $\mathrm{F}=\operatorname{str} 2 \operatorname{double}\left(\operatorname{get}\left(\mathrm{F}_{-}^{-} \mathrm{kR}\right.\right.$, 'String')); switch $\mathrm{kR}$ check. Value

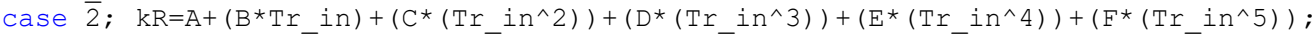

end

case $3 ; \mathrm{kR}=\mathrm{A}^{\star}\left(\mathrm{B}^{\wedge}\left(\mathrm{C}^{\star} \operatorname{Tr}\right.\right.$ in $\left.)\right)$

end

if Ts in>273.15

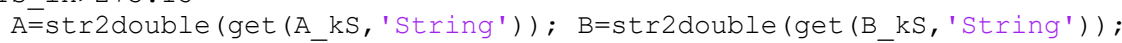
$\mathrm{C}=$ str2double (get (C kS, 'String')); $\mathrm{D}=$ str2double (get (D kS, 'String'));

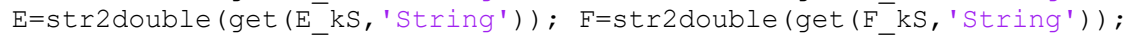
switch kS check. Value

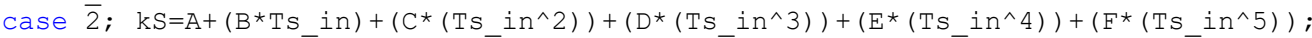
end case $3 ; \mathrm{kS}=\mathrm{A}^{*}\left(\mathrm{~B}^{\wedge}\left(\mathrm{C}^{\star} \mathrm{TS}\right.\right.$ in $\left.)\right)$

end

$q(1)=($ Tc1_limit-Tinf_max $) /(\operatorname{Rinf}+(\mathrm{Rc} 1 / \mathrm{kCl}))$; \%Heat generated by capsule 1

$\mathrm{q}(2)=(\mathrm{Tc} 2$ limit-Tinf $\max ) /($ Rinf+(Rc1/kC1) $+(\mathrm{RGap} / \mathrm{kGap})+(\mathrm{Rc} 2 / \mathrm{kC2}))$; $\%$ Heat generated by capsule 2 $\mathrm{q}(3)=\left(\mathrm{Ts} \_\bar{l}\right.$ imit-Tinf_max $) /(\operatorname{Rinf}+(\operatorname{Rc} 1 / \mathrm{kCl})+(\mathrm{RGap} / \mathrm{kGap})+(\mathrm{Rc} 2 / \mathrm{kC} 2)+(\mathrm{RBond} / \mathrm{kBond})+(\mathrm{Rr} / \mathrm{kR})+(\mathrm{Rs} / \mathrm{kS}))$; \%Heat generated by sample 
$\mathrm{Q}=\min (\mathrm{q})$;

end 


\subsection{PROP.m}

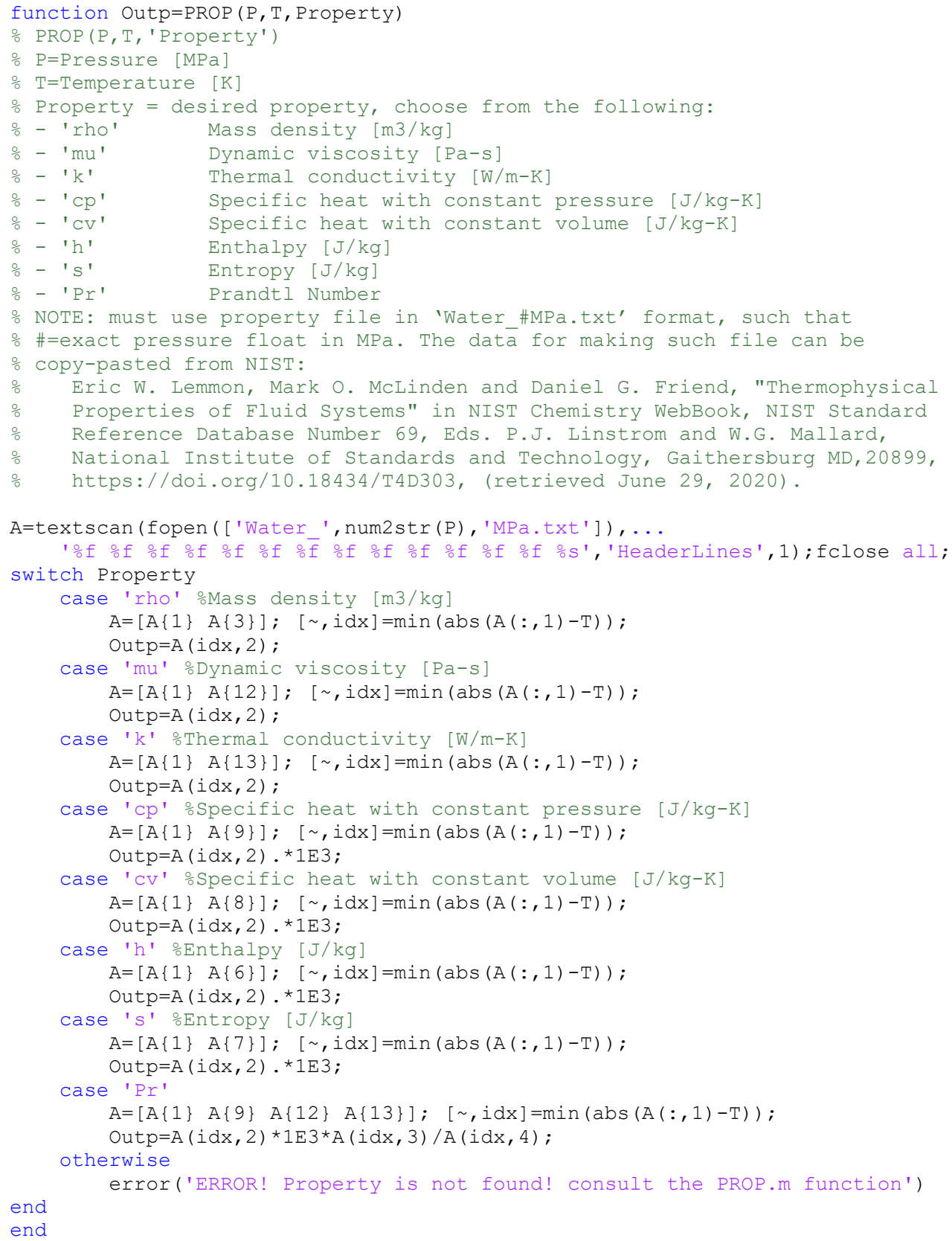




\subsection{Water_2.5MPa.txt [From Reference 19]}

\begin{tabular}{|c|c|c|c|c|c|c|c|}
\hline $\begin{array}{l}\text { Tempera } \\
\left(\mathrm{J} / \mathrm{g}^{\star} \mathrm{K}\right)\end{array}$ & $\begin{aligned} \text { ure } & (\mathrm{K}) \\
\mathrm{Cp} & (\mathrm{J}\end{aligned}$ & $\begin{array}{l}\text { Pressu } \\
\left.g^{\star} \mathrm{K}\right) \quad \mathrm{S}\end{array}$ & $\begin{array}{cc}\text { (MPa) } & \text { Der } \\
\text { and Spd. } & (\mathrm{m}\end{array}$ & ity $(\mathrm{kg}$ & $\begin{array}{l}\text { (m3) Vo- } \\
\text { ale-Thon }\end{array}$ & $\begin{array}{ll}\text { ame } & (\mathrm{m} 3 / \mathrm{k} \\
\text { son } & (\mathrm{K} / \mathrm{MP}\end{array}$ & \\
\hline 274.00 & 2.5000 & 1001.1 & 0.00099890 & 3.5781 & 6.0754 & 0.013073 & $4.2 \cdot \ln (2)$ \\
\hline 275.66 & 2.5000 & 1001.2 & 0.00099885 & 10.555 & 13.052 & 0.038459 & \\
\hline 277.32 & 2.5000 & 1001.2 & 0.00099884 & 17.525 & 20.022 & 0.063666 & \\
\hline 278.98 & 2.5000 & 1001.1 & 0.00099888 & 24.488 & 26.985 & 0.088701 & \\
\hline 280.64 & 2.5000 & 1001.0 & 0.00099896 & 31.446 & 33.943 & 0.11357 & 4.1880 \\
\hline 282.30 & 2.5000 & 1000.9 & 0.00099908 & 38.399 & 40.896 & 0.13827 & 4.1834 \\
\hline 283.96 & 2.5000 & 1000.8 & 0.00099923 & 45.347 & 47.846 & 0.16282 & 4.1785 \\
\hline 285.62 & 2.5000 & 1000.6 & 0.00099943 & 52.292 & 54.791 & 0.18720 & 4.1735 \\
\hline 287.28 & 2.5000 & 1000.4 & 0.00099965 & 59.234 & 61.733 & 0.21144 & 4.1682 \\
\hline 288.94 & 2.5000 & 1000.1 & 0.00099991 & 66.173 & 68.673 & 0.23553 & 4.1628 \\
\hline 290.60 & 2.5000 & 999.80 & 0.0010002 & 73.109 & 75.610 & 0.25947 & 4.1571 \\
\hline 292.26 & 2.5000 & 999.49 & 0.0010005 & 80.043 & 82.545 & 0.28326 & 4.1513 \\
\hline 293.92 & 2.5000 & 999.14 & 0.0010009 & 86.976 & 89.478 & 0.30692 & 4.1454 \\
\hline 295.58 & 2.5000 & 998.76 & 0.0010012 & 93.907 & 96.410 & 0.33044 & 4.1393 \\
\hline 297.24 & 2.5000 & 998.36 & 0.0010016 & 100.84 & 103.34 & 0.35382 & 4.1330 \\
\hline 298.90 & 2.5000 & 997.93 & 0.0010021 & 107.76 & 110.27 & 0.37707 & 4.1266 \\
\hline 300.56 & 2.5000 & 997.47 & 0.0010025 & 114.69 & 117.20 & 0.40018 & 4.1201 \\
\hline 302.22 & 2.5000 & 997.00 & 0.0010030 & 121.62 & 124.13 & 0.42317 & 4.1134 \\
\hline 303.88 & 2.5000 & 996.49 & 0.0010035 & 128.55 & 131.05 & 0.44603 & 4.1066 \\
\hline 305.54 & 2.5000 & 995.96 & 0.0010041 & 135.47 & 137.98 & 0.46876 & 4.0997 \\
\hline 307.20 & 2.5000 & 995.41 & 0.0010046 & 142.40 & 144.91 & 0.49138 & 4.0927 \\
\hline 308.86 & 2.5000 & 994.84 & 0.0010052 & 149.32 & 151.84 & 0.51387 & 4.0856 \\
\hline 310.52 & 2.5000 & 994.25 & 0.0010058 & 156.25 & 158.76 & 0.53623 & 4.0783 \\
\hline 312.18 & 2.5000 & 993.64 & 0.0010064 & 163.18 & 165.69 & 0.55849 & 4.0709 \\
\hline 313.84 & 2.5000 & 993.00 & 0.0010070 & 170.10 & 172.62 & 0.58062 & 4.0635 \\
\hline 315.50 & 2.5000 & 992.35 & 0.0010077 & 177.03 & 179.55 & 0.60264 & 4.0559 \\
\hline 317.16 & 2.5000 & 991.67 & 0.0010084 & 183.96 & 186.48 & 0.62454 & 4.0483 \\
\hline 318.82 & 2.5000 & 990.98 & 0.0010091 & 190.88 & 193.41 & 0.64633 & 4.0406 \\
\hline 320.48 & 2.5000 & 990.26 & 0.0010098 & 197.81 & 200.34 & 0.66801 & 4.0328 \\
\hline 322.14 & 2.5000 & 989.53 & 0.0010106 & 204.74 & 207.27 & 0.68959 & 4.0249 \\
\hline 323.80 & 2.5000 & 988.78 & 0.0010113 & 211.67 & 214.20 & 0.71105 & 4.0169 \\
\hline 325.46 & 2.5000 & 988.02 & 0.0010121 & 218.60 & 221.13 & 0.73240 & 4.0089 \\
\hline 327.12 & 2.5000 & 987.23 & 0.0010129 & 225.53 & 228.07 & 0.75365 & 4.0008 \\
\hline 328.78 & 2.5000 & 986.43 & 0.0010138 & 232.47 & 235.00 & 0.77480 & 3.9927 \\
\hline 330.44 & 2.5000 & 985.61 & 0.0010146 & 239.40 & 241.94 & 0.79584 & 3.9845 \\
\hline 332.10 & 2.5000 & 984.78 & 0.0010155 & 246.34 & 248.87 & 0.81678 & 3.9762 \\
\hline 333.76 & 2.5000 & 983.93 & 0.0010163 & 253.27 & 255.81 & 0.83762 & 3.9679 \\
\hline 335.42 & 2.5000 & 983.06 & 0.0010172 & 260.21 & 262.75 & 0.85836 & 3.9595 \\
\hline 337.08 & 2.5000 & 982.18 & 0.0010181 & 267.15 & 269.69 & 0.87900 & 3.9511 \\
\hline 338.74 & 2.5000 & 981.28 & 0.0010191 & 274.09 & 276.63 & 0.89955 & 3.9427 \\
\hline 340.40 & 2.5000 & 980.37 & 0.0010200 & 281.03 & 283.58 & 0.92000 & 3.9342 \\
\hline 342.06 & 2.5000 & 979.44 & 0.0010210 & 287.97 & 290.52 & 0.94035 & 3.9257 \\
\hline 343.72 & 2.5000 & 978.50 & 0.0010220 & 294.91 & 297.47 & 0.96061 & 3.9172 \\
\hline 345.38 & 2.5000 & 977.54 & 0.0010230 & 301.86 & 304.42 & 0.98077 & 3.9086 \\
\hline 347.04 & 2.5000 & 976.56 & 0.0010240 & 308.81 & 311.37 & 1.0008 & 3.9001 \\
\hline 348.70 & 2.5000 & 975.58 & 0.0010250 & 315.76 & 318.32 & 1.0208 & 3.8915 \\
\hline 350.36 & 2.5000 & 974.58 & 0.0010261 & 322.71 & 325.27 & 1.0407 & 3.8828 \\
\hline 352.02 & 2.5000 & 973.56 & 0.0010272 & 329.66 & 332.23 & 1.0605 & 3.8742 \\
\hline 353.68 & 2.5000 & 972.53 & 0.0010282 & 336.62 & 339.19 & 1.0803 & 3.8656 \\
\hline 355.34 & 2.5000 & 971.49 & 0.0010293 & 343.57 & 346.15 & 1.0999 & 3.8569 \\
\hline 357.00 & 2.5000 & 970.43 & 0.0010305 & 350.53 & 353.11 & 1.1194 & 3.8483 \\
\hline 358.66 & 2.5000 & 969.36 & 0.0010316 & 357.49 & 360.07 & 1.1389 & 3.8397 \\
\hline 360.32 & 2.5000 & 968.28 & 0.0010328 & 364.46 & 367.04 & 1.1583 & 3.8310 \\
\hline 361.98 & 2.5000 & 967.18 & 0.0010339 & 371.42 & 374.01 & 1.1776 & 3.8224 \\
\hline 363.64 & 2.5000 & 966.08 & 0.0010351 & 378.39 & 380.98 & 1.1968 & 3.8137 \\
\hline 365.30 & 2.5000 & 964.95 & 0.0010363 & 385.36 & 387.95 & 1.2159 & 3.8051 \\
\hline 366.96 & 2.5000 & 963.82 & 0.0010375 & 392.33 & 394.93 & 1.2350 & 3.7965 \\
\hline 368.62 & 2.5000 & 962.67 & 0.0010388 & 399.31 & 401.91 & 1.2539 & 3.7879 \\
\hline 370.28 & 2.5000 & 961.51 & 0.0010400 & 406.29 & 408.89 & 1.2728 & 3.7793 \\
\hline 371.94 & 2.5000 & 960.34 & 0.0010413 & 413.27 & 415.88 & 1.2917 & 3.7707 \\
\hline 373.60 & 2.5000 & 959.15 & 0.0010426 & 420.26 & 422.86 & 1.3104 & 3.7622 \\
\hline 375.26 & 2.5000 & 957.95 & 0.0010439 & 427.25 & 429.85 & 1.3291 & 3.7536 \\
\hline 376.92 & 2.5000 & 956.74 & 0.0010452 & 434.24 & 436.85 & 1.3477 & 3.7451 \\
\hline 378.58 & 2.5000 & 955.52 & 0.0010466 & 441.23 & 443.85 & 1.3662 & 3.7366 \\
\hline 380.24 & 2.5000 & 954.28 & 0.0010479 & 448.23 & 450.85 & 1.3847 & 3.7281 \\
\hline 381.90 & 2.5000 & 953.03 & 0.0010493 & 455.23 & 457.85 & 1.4030 & 3.7197 \\
\hline 383.56 & 2.5000 & 951.77 & 0.0010507 & 462.24 & 464.86 & 1.4214 & 3.7113 \\
\hline 385.22 & 2.5000 & 950.50 & 0.0010521 & 469.24 & 471.88 & 1.4396 & 3.7029 \\
\hline 386.88 & 2.5000 & 949.22 & 0.00 & 476.26 & 478.89 & 1.4578 & 3.6946 \\
\hline 388.54 & 2.5000 & 947.92 & 0.0010549 & 483.27 & 485.91 & 1.4759 & 3.6862 \\
\hline 390.20 & 2.5000 & 946.61 & 0.0010564 & 490.30 & 492.94 & 1.4939 & 3.6779 \\
\hline 391.86 & 2.5000 & 945.29 & 0.0010579 & 497.32 & 499.97 & 1.5119 & 3.6697 \\
\hline 393.52 & 2.5000 & 943.96 & 0.0010594 & 504.35 & 507.00 & 1.5298 & 3.6615 \\
\hline 395.18 & 2.5000 & 942.61 & 0.0010609 & 511.38 & 514.04 & 1.5477 & 3.6533 \\
\hline 396.84 & 2.5000 & 941.26 & 0.0010624 & 518.42 & 521.08 & 1.5654 & 3.6451 \\
\hline 398.50 & 2.5000 & 939.89 & 0.0010640 & 525.47 & 528.13 & 1.5832 & 3.6370 \\
\hline 400.16 & 2.5000 & 938.51 & 0.0010655 & 532.51 & 535.18 & 1.6008 & 3.6289 \\
\hline 401.82 & 2.5000 & 937.11 & 0.0010671 & 539.57 & 542.24 & 1.6184 & 3.6209 \\
\hline 403.48 & 2.5000 & 935.71 & 0.0010687 & 546.63 & 549.30 & 1.6360 & 3.6129 \\
\hline 405.14 & 2.5000 & 934.29 & 0.0010703 & 553.69 & 556.37 & 1.6534 & 3.6049 \\
\hline 406.80 & 2.5000 & 932.87 & 0.0010720 & 560.76 & 563.44 & 1.6709 & 3.5970 \\
\hline 408.46 & 2.5000 & 931.43 & 0.0010736 & 567.83 & 570.52 & 1.6882 & 3.5891 \\
\hline 410.12 & 2.5000 & 929.97 & 0.0010753 & 574.91 & 577.60 & 1.7055 & 3.5813 \\
\hline 411.78 & 2.5000 & 928.51 & 0.0010770 & 582.00 & 584.69 & 1.7228 & 3.5735 \\
\hline 413.44 & 2.5000 & 927.03 & 0.0010787 & 589.09 & 591.79 & 1.7400 & 3.5658 \\
\hline 415.10 & 2.5000 & 925.54 & 0.0010804 & 596.19 & 598.89 & 1.7571 & 3.5581 \\
\hline 416.76 & 2.5000 & 924.04 & 0.0010822 & 3.30 & 606.00 & 1.7742 & 3.5504 \\
\hline 418.42 & 2.5000 & 922.53 & 0.0010840 & 610.41 & 613.12 & 1.7913 & 3.5428 \\
\hline 420.08 & 2.5000 & 921.01 & 0.0010858 & 617.53 & 620.24 & 1.8083 & 3.5352 \\
\hline 421.74 & 2.5000 & 919.47 & 0.0010876 & 624.65 & 627.37 & 1.8252 & 3.5277 \\
\hline
\end{tabular}


$\begin{array}{llll}423.40 & 2.5000 & 917.92 & 0.0010894\end{array}$ $\begin{array}{llll}425.06 & 2.5000 & 916.36 & 0.0010913 \\ 426.72 & 2.5000 & 914.78 & 0.0010932\end{array}$ $\begin{array}{lllll}428.38 & 2.5000 & 913.20 & 0.0010951\end{array}$ $\begin{array}{llll}430.04 & 2.5000 & 911.60 & 0.0010970\end{array}$ $\begin{array}{llll}431.70 & 2.5000 & 909.99 & 0.0010989\end{array}$ $\begin{array}{lllll}433.36 & 2.5000 & 908.36 & 0.0011009\end{array}$ $\begin{array}{llll}435.02 & 2.5000 & 906.73 & 0.0011029\end{array}$ $\begin{array}{llll}436.68 & 2.5000 & 905.08 & 0.0011049\end{array}$ $\begin{array}{llll}438.34 & 2.5000 & 903.42 & 0.0011069 \\ 440.00 & 2.5000 & 901.74 & 0.0011090\end{array}$ $\begin{array}{llll}441.66 & 2.5000 & 900.05 & 0.0011110\end{array}$ $\begin{array}{llll}443.32 & 2.5000 & 898.35 & 0.0011132\end{array}$ $\begin{array}{llll}444.98 & 2.5000 & 896.64 & 0.0011153 \\ 446.64 & 2.5000 & 894.91 & 0.0011174\end{array}$ $\begin{array}{llll}448.30 & 2.5000 & 893.17 & 0.0011196\end{array}$ $\begin{array}{llll}449.96 & 2.5000 & 891.41 & 0.0011218\end{array}$ $\begin{array}{lllll}451.62 & 2.5000 & 889.64 & 0.0011240\end{array}$ $\begin{array}{llll}453.28 & 2.5000 & 887.86 & 0.0011263\end{array}$ $\begin{array}{llll}454.94 & 2.5000 & 886.06 & 0.0011286 \\ 456.60 & 2.5000 & 884.25 & 0.0011309\end{array}$ $\begin{array}{llll}458.26 & 2.5000 & 882.42 & 0.0011332\end{array}$ $\begin{array}{llll}459.92 & 2.5000 & 880.58 & 0.0011356\end{array}$ $\begin{array}{llll}461.58 & 2.5000 & 878.73 & 0.0011380\end{array}$ $\begin{array}{llll}463.24 & 2.5000 & 876.86 & 0.0011404 \\ 464.90 & 2.5000 & 874.97 & 0.0011429\end{array}$ $\begin{array}{llll}464.90 & 2.5000 & 874.97 & 0.0011429 \\ 466.56 & 2.5000 & 873.07 & 0.0011454\end{array}$ $\begin{array}{llll}468.22 & 2.5000 & 871.16 & 0.0011479\end{array}$ $\begin{array}{llll}469.88 & 2.5000 & 869.23 & 0.0011504\end{array}$ $\begin{array}{lllll}471.54 & 2.5000 & 867.28 & 0.0011530\end{array}$ $\begin{array}{llll}473.20 & 2.5000 & 865.32 & 0.0011556\end{array}$ $\begin{array}{llll}474.86 & 2.5000 & 863.34 & 0.0011583\end{array}$ $\begin{array}{llll}476.52 & 2.5000 & 861.34 & 0.0011610 \\ 478.18 & 2.5000 & 859.33 & 0.0011637\end{array}$ $\begin{array}{llll}478.18 & 2.5000 & 859.33 & 0.0011637 \\ 479.84 & 2.5000 & 857.30 & 0.0011665\end{array}$ $\begin{array}{llll}481.50 & 2.5000 & 855.25 & 0.0011692\end{array}$ $\begin{array}{llll}483.16 & 2.5000 & 853.19 & 0.0011721 \\ 484.82 & 2.5000 & 851.11 & 0.0011749\end{array}$ $\begin{array}{llll}484.82 & 2.5000 & 851.11 & 0.0011749 \\ 486.48 & 2.5000 & 849.01 & 0.0011778\end{array}$ $\begin{array}{lllll}486.48 & 2.5000 & 849.01 & 0.0011778\end{array}$ $\begin{array}{llll}488.14 & 2.5000 & 846.89 & 0.0011808 \\ 489.80 & 2.5000 & 844.75 & 0.0011838\end{array}$ $\begin{array}{llll}489.80 & 2.5000 & 844.75 & 0.0011838 \\ 491.46 & 2.5000 & 842.60 & 0.0011868\end{array}$ $\begin{array}{llll}493.12 & 2.5000 & 840.42 & 0.0011899\end{array}$ $\begin{array}{llll}494.78 & 2.5000 & 838.22 & 0.0011930\end{array}$ $\begin{array}{llll}496.44 & 2.5000 & 836.01 & 0.0011962\end{array}$ $\begin{array}{llll}497.10 & 2.5000 & 835.12 & 0.0011974 \\ 497.10 & 2.5000 & 12.508 & 0.079949\end{array}$ $\begin{array}{llll}497.10 & 2.5000 & 12.508 & 0.079949 \\ 498.10 & 2.5000 & 12.462 & 0.080245\end{array}$ $\begin{array}{lllll}499.76 & 2.5000 & 12.387 & 0.080731\end{array}$ $\begin{array}{llll}501.42 & 2.5000 & 12.314 & 0.081209\end{array}$ $\begin{array}{llll}503.08 & 2.5000 & 12.243 & 0.081682\end{array}$ $\begin{array}{llll}504.74 & 2.5000 & 12.173 & 0.082149\end{array}$ $\begin{array}{llll}506.40 & 2.5000 & 12.105 & 0.082611\end{array}$ $\begin{array}{lllll}508.06 & 2.5000 & 12.038 & 0.083069\end{array}$ $\begin{array}{llll}509.72 & 2.5000 & 11.973 & 0.083522\end{array}$ $\begin{array}{llll}511.38 & 2.5000 & 11.909 & 0.083971\end{array}$ $\begin{array}{llll}513.04 & 2.5000 & 11.846 & 0.084416\end{array}$ $\begin{array}{llll}514.70 & 2.5000 & 11.785 & 0.084857\end{array}$ $\begin{array}{llll}516.36 & 2.5000 & 11.724 & 0.085295 \\ 518.02 & 2.5000 & 11.665 & 0.085729\end{array}$ $\begin{array}{lllll}519.68 & 2.5000 & 11.606 & 0.086161\end{array}$ $\begin{array}{llll}521.34 & 2.5000 & 11.549 & 0.086589\end{array}$ $\begin{array}{llll}523.00 & 2.5000 & 11.492 & 0.08701\end{array}$ $\begin{array}{llll}524.66 & 2.5000 & 11.437 & 0.087438\end{array}$ $\begin{array}{llll}526.32 & 2.5000 & 11.382 & 0.087858\end{array}$ $\begin{array}{llll}527.98 & 2.5000 & 11.328 & 0.088276\end{array}$ $\begin{array}{llll}529.64 & 2.5000 & 11.275 & 0.08869\end{array}$ $\begin{array}{llll}531.30 & 2.5000 & 11.223 & 0.089104 \\ 532.96 & 2.5000 & 11.171 & 0.089515\end{array}$ $\begin{array}{llll}532.96 & 2.5000 & 11.171 & 0.089515 \\ 534.62 & 2.5000 & 11.121 & 0.089924\end{array}$ $\begin{array}{llll}534.62 & 2.5000 & 11.121 & 0.089924 \\ 536.28 & 2.5000 & 11.070 & 0.090330\end{array}$ $\begin{array}{llll}537.94 & 2.5000 & 11.021 & 0.090735\end{array}$ $\begin{array}{llll}539.60 & 2.5000 & 10.972 & 0.091137\end{array}$ $\begin{array}{llll}541.26 & 2.5000 & 10.924 & 0.091538\end{array}$ $\begin{array}{lllll}542.92 & 2.5000 & 10.877 & 0.091937\end{array}$ $\begin{array}{lllll}544.58 & 2.5000 & 10.830 & 0.092334\end{array}$ $\begin{array}{llll}546.24 & 2.5000 & 10.784 & 0.092729\end{array}$ $\begin{array}{llll}4.90 & 2.5000 & 10.738 & 0.093123\end{array}$ $\begin{array}{llll}549.56 & 2.5000 & 10.693 & 0.093515\end{array}$ $\begin{array}{llll}551.22 & 2.5000 & 10.649 & 0.09390\end{array}$ $\begin{array}{llll}552.88 & 2.5000 & 10.605 & 0.094295\end{array}$ $\begin{array}{llll}554.54 & 2.5000 & 10.562 & 0.094682 \\ 556.20 & 2.5000 & 10.519 & 0.095068\end{array}$ $\begin{array}{llll}556.20 & 2.5000 & 10.519 & 0.095068\end{array}$ $\begin{array}{llll}557.86 & 2.5000 & 10.476 & 0.095453\end{array}$ $\begin{array}{lll}559.52 & 2.5000 & 10.434\end{array}$ $\begin{array}{llll}561.18 & 2.5000 & 10.393 & 0.096218\end{array}$ $\begin{array}{llll}562.84 & 2.5000 & 10.352 & 0.096599\end{array}$ $\begin{array}{lllll}564.50 & 2.5000 & 10.312 & 0.096978\end{array}$ $\begin{array}{llll}566.16 & 2.5000 & 10.272 & 0.097357\end{array}$ $\begin{array}{llll}567.82 & 2.5000 & 10.232 & 0.09773\end{array}$ $\begin{array}{llll}569.48 & 2.5000 & 10.193 & 0.09811\end{array}$ $\begin{array}{llll}571.14 & 2.5000 & 10.154 & 0.098484 \\ 572.80 & 2.5000 & 10.116 & 0.098858\end{array}$ $\begin{array}{llll}572.80 & 2.5000 & 10.116 & 0.098858 \\ 574.46 & 2.5000 & 10.078 & 0.099231\end{array}$ $\begin{array}{lllll}576.12 & 2.5000 & 10.040 & 0.099602\end{array}$ $\begin{array}{lllllll}631.78 & 634.51 & 1.8421 & 3.5202 & 4.3012 & 1470.8 & -0.14382\end{array}$ $\begin{array}{lllllll}638.92 & 641.65 & 1.8589 & 3.5128 & 4.3056 & 1467.2 & -0.14243\end{array}$ $\begin{array}{lllllll}646.07 & 648.80 & 1.8757 & 3.5054 & 4.3101 & 1463.5 & -0.14101\end{array}$ $\begin{array}{lllllll}653.22 & 655.96 & 1.8925 & 3.4980 & 4.3147 & 1459.7 & -0.13958\end{array}$ $\begin{array}{lllllll}660.38 & 663.13 & 1.9092 & 3.4907 & 4.3195 & 1455.9 & -0.13812\end{array}$ $\begin{array}{lllllll}667.55 & 670.30 & 1.9258 & 3.4834 & 4.3243 & 1452.1 & -0.13664\end{array}$ $\begin{array}{lllllll}674.73 & 677.48 & 1.9424 & 3.4762 & 4.3293 & 1448.1 & -0.13514\end{array}$ $\begin{array}{llllllll}681.92 & 684.67 & 1.9590 & 3.4691 & 4.3344 & 1444.1 & -0.13362\end{array}$ $\begin{array}{lllllll}689.11 & 691.87 & 1.9755 & 3.4619 & 4.3396 & 1440.1 & -0.13207\end{array}$ $\begin{array}{lllllll}696.31 & 699.08 & 1.9920 & 3.4549 & 4.3449 & 1436.0 & -0.13049\end{array}$ $\begin{array}{lllllll}703.52 & 706.30 & 2.0084 & 3.4478 & 4.3503 & 1431.8 & -0.12889\end{array}$ $\begin{array}{lllllll}710.75 & 713.52 & 2.0248 & 3.4409 & 4.3559 & 1427.5 & -0.12726\end{array}$ $\begin{array}{lllllll}717.98 & 720.76 & 2.0411 & 3.4339 & 4.3616 & 1423.2 & -0.12561 \\ 725.22 & 728.00 & 2.0575 & 3.4270 & 4.3675 & 1418.9 & -0.12393\end{array}$ $\begin{array}{lllllll}725.22 & 728.00 & 2.0575 & 3.4270 & 4.3675 & 1418.9 & -0.12393 \\ 732.47 & 735.26 & 2.0737 & 3.4202 & 4.3734 & 1414.5 & -0.12222\end{array}$ $\begin{array}{lllllll}739.73 & 742.52 & 2.0900 & 3.4134 & 4.3796 & 1410.0 & -0.12048\end{array}$ $\begin{array}{lllllll}746.99 & 749.80 & 2.1062 & 3.4067 & 4.3858 & 1405.4 & -0.11871 \\ 754.28 & 757.09 & 2.1223 & 3.4000 & 4.3922 & 1400.8 & -0.11691\end{array}$ $\begin{array}{lllllll}761.57 & 764.38 & 2.1385 & 3.3933 & 4.3988 & 1396.2 & -0\end{array}$ $\begin{array}{lllllll}761.57 & 764.38 & 2.1385 & 3.3933 & 4.3988 & 1396.2 & -0.11508 \\ 768.87 & 771.69 & 2.1545 & 3.3867 & 4.4055 & 1391.4 & -0.11322\end{array}$ $\begin{array}{lllllll}776.18 & 779.01 & 2.1706 & 3.3801 & 4.4124 & 1386.6 & -0.11132\end{array}$ $\begin{array}{lllllll}783.51 & 786.34 & 2.1866 & 3.3736 & 4.4194 & 1381.8 & -0.10939\end{array}$ $\begin{array}{lllllll}790.84 & 793.68 & 2.2026 & 3.3672 & 4.4266 & 1376.9 & -0.10742\end{array}$ $\begin{array}{lllllll}798.19 & 801.03 & 2.2186 & 3.3607 & 4.4340 & 1371.9 & -0.10541\end{array}$ $\begin{array}{lllllll}805.55 & 808.40 & 2.2345 & 3.3544 & 4.4415 & 1366.9 & -0.10337 \\ 812.92 & 815.78 & 2.2504 & 3.3481 & 4.4492 & 1361.8 & -0.10129\end{array}$ $\begin{array}{lllllll}812.92 & 815.78 & 2.2504 & 3.3481 & 4.4492 & 1361.8 & -0.10129\end{array}$ $\begin{array}{lllllll}820.31 & 823.17 & 2.2663 & 3.3418 & 4.4571 & 1356.6 & -0.099168 \\ 827.71 & 830.58 & 2.2821 & 3.3355 & 4.4652 & 1351.4 & -0.097005\end{array}$ $\begin{array}{llllllll}835.12 & 838.00 & 2.2979 & 3.3294 & 4.4735 & 1346.1 & -0.094800\end{array}$ $\begin{array}{lllllll}842.55 & 845.43 & 2.3137 & 3.3232 & 4.4819 & 1340.8 & -0.092551\end{array}$ $\begin{array}{lllllll}849.99 & 852.88 & 2.3295 & 3.3171 & 4.4906 & 1335.3 & -0.090256\end{array}$ $\begin{array}{llllllll}857.44 & 860.34 & 2.3452 & 3.3111 & 4.4995 & 1329.9 & -0.087916\end{array}$ $\begin{array}{llllllll}864.91 & 867.82 & 2.3610 & 3.3051 & 4.5086 & 1324.3 & -0.085526\end{array}$ $\begin{array}{llllllll}872.40 & 875.31 & 2.3767 & 3.2992 & 4.5179 & 1318.7 & -0.083088 \\ 879.90 & 882.82 & 2.3923 & 3.2933 & 4.5275 & 1313.1 & -0.080598\end{array}$

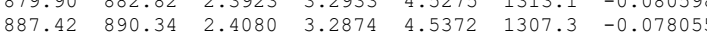
$\begin{array}{llllllll}894.95 & 897.88 & 2.4236 & 3.2816 & 4.5473 & 1301.5 & -0.075458 \\ 902.50 & 905.44 & 2.4392 & 3.2759 & 4.5575 & 1295.7 & -0.072804\end{array}$ $\begin{array}{llllllll}910.07 & 913.01 & 2.4548 & 3.2702 & 4.5681 & 1289.8 & -0.070092\end{array}$ $\begin{array}{llllllll}917.65 & 920.60 & 2.4704 & 3.2645 & 4.5789 & 1283.8 & -0.067320\end{array}$ $\begin{array}{lllllll}925.25 & 928.21 & 2.4860 & 3.2589 & 4.5900 & 1277.7 & -0.064485\end{array}$ $\begin{array}{llllllll}932.87 & 935.84 & 2.5015 & 3.2534 & 4.6013 & 1271.6 & -0.061586\end{array}$ $\begin{array}{lllllll}940.51 & 943.49 & 2.5171 & 3.2479 & 4.6130 & 1265.4 & -0.058620\end{array}$ $\begin{array}{lllllll}948.17 & 951.16 & 2.5326 & 3.2424 & 4.6249 & 1259.1 & -0.055584\end{array}$ $\begin{array}{lllllll}955.85 & 958.84 & 2.5481 & 3.2370 & 4.6372 & 1252.8 & -0.052477\end{array}$ $\begin{array}{lllllll}958.91 & 961.91 & 2.5543 & 3.2349 & 4.6422 & 1250.2 & -0.051221\end{array}$ $\begin{array}{lllllll}2602.1 & 2801.9 & 6.2558 & 2.2483 & 3.4050 & 504.67 & 19.935 \\ 2604.7 & 2805.3 & 6.2626 & 2.2228 & 3.3595 & 505.86 & 19.794\end{array}$ $\begin{array}{llllllllll}2604.7 & 2805.3 & 6.2626 & 2.2228 & 3.3595 & 505.86 & 19.794 & 1.6550 \mathrm{e}-05 & 0.045025 & \text { vapor }\end{array}$ $\begin{array}{llllllllll}2609.0 & 2810.8 & 6.2736 & 2.1849 & 3.2915 & 507.76 & 19.563 & 1.6597 e-05 & 0.045004 & \text { vapor } \\ \end{array}$ $\begin{array}{llllllllll}2613.2 & 2816.2 & 6.2844 & 2.1517 & 3.2313 & 509.59 & 19.335 & 1.6754 \mathrm{e}-05 & 0.044951 & \text { vapor }\end{array}$ $\begin{array}{llllllllll}2617.4 & 2821.6 & 6.2950 & 2.1224 & 3.1776 & 511.35 & 19.111 & 1.6832 \mathrm{e}-05 & 0.044935 & \text { vapor }\end{array}$ $\begin{array}{llllllllll}2621.4 & 2826.8 & 6.3054 & 2.0963 & 3.1293 & 513.05 & 18.891 & 1.6910 \mathrm{e}-05 & 0.044925 & \text { vapor }\end{array}$ $\begin{array}{llllllllll}2625.4 & 2832.0 & 6.3156 & 2.0729 & 3.0856 & 514.70 & 18.675 & 1.6987 \mathrm{e}-05 & 0.044921 & \text { vapor }\end{array}$ $\begin{array}{llllllllll}2629.4 & 2837.0 & 6.3257 & 2.0517 & 3.0457 & 516.31 & 18.463 & 1.7065 \mathrm{e}-05 & 0.044922 & \text { vapor }\end{array}$ $\begin{array}{llllllllll}2633.3 & 2842.1 & 6.3355 & 2.0325 & 3.0091 & 517.88 & 18.254 & 1.7142 \mathrm{e}-05 & 0.044929 & \text { vapor }\end{array}$ $\begin{array}{llllllllll}2637.1 & 2847.0 & 6.3453 & 2.0149 & 2.9752 & 519.41 & 18.049 & 1.7219 \mathrm{e}-05 & 0.044942 & \text { vapor }\end{array}$ $\begin{array}{llllllllll}2640.9 & 2851.9 & 6.3548 & 1.9986 & 2.9438 & 520.92 & 17.848 & 1.7297 e-05 & 0.044959 & \text { vapor }\end{array}$ \begin{tabular}{llllllllll}
2644.7 & 2856.8 & 6.3643 & 1.9836 & 2.9146 & 522.39 & 17.650 & $1.7374 \mathrm{e}-05$ & 0.044981 & vapor \\
\hline
\end{tabular} $\begin{array}{llllllllll}2648.4 & 2861.6 & 6.3736 & 1.9696 & 2.8871 & 523.84 & 17.455 & 1.7450 e-05 & 0.045007 & \text { vapor } \\ 2652.1 & 2866.4 & 6.3829 & 1.9565 & 2.8614 & 525.27 & 17.264 & 1.7527 e-05 & 0.045038 & \text { vapor }\end{array}$ $\begin{array}{llllllllll}2652.1 & 2866.4 & 6.3829 & 1.9565 & 2.8614 & 525.27 & 17.264 & 1.7527 e-05 & 0.045038 & \text { vapor } \\ 2655.7 & 2871.1 & 6.3920 & 1.9443 & 2.8370 & 526.68 & 17.075 & 1.7604 e-05 & 0.045073 & \text { vapor }\end{array}$ $\begin{array}{llllllllll}2655.7 & 2871.1 & 6.3920 & 1.9443 & 2.8370 & 526.68 & 17.075 & 1.76040-05 & 0.045073 \\ 2659.3 & 2875.8 & 6.4010 & 1.9327 & 2.8141 & 528.06 & 16.890 & 1.7680 e-05 & 0.045112\end{array}$ $\begin{array}{llllllllll}2662.9 & 2880.5 & 6.4099 & 1.9218 & 2.7923 & 529.43 & 16.708 & 1.7757 \mathrm{e}-05 & 0.045155 & \text { vapor }\end{array}$ $\begin{array}{llllllllll}2666.5 & 2885.1 & 6.4187 & 1.9115 & 2.7715 & 530.78 & 16.528 & 1.7833 e-05 & 0.045202 & \text { vapor }\end{array}$

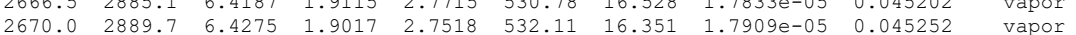
$\begin{array}{llllllllll}2670.0 & 2889.7 & 6.4275 & 1.9017 & 2.7518 & 532.11 & 16.351 & 1.7909 \mathrm{e}-05 & 0.045252 & \text { vapor } \\ 2673.5 & 2894.2 & 6.4361 & 1.8924 & 2.7330 & 533.43 & 16.177 & 1.7985 \mathrm{e}-05 & 0.045306 & \text { vapor }\end{array}$ $\begin{array}{llllllllll}2677.0 & 2898.7 & 6.4446 & 1.8836 & 2.7150 & 534.74 & 16.006 & 1.8061 \mathrm{e}-05 & 0.045364 & \text { vapor } \\ 2680.5 & 2903.2 & 6.4531 & 1.8751 & 2.6978 & 536.03 & 15.837 & 1.8137 \mathrm{p}-05 & 0.045424 & \text { vapor }\end{array}$ $\begin{array}{llllllllll}2677.0 & 2898.7 & 6.4446 & 1.8836 & 2.7150 & 534.74 & 16.006 & 1.8061 \mathrm{e}-05 & 0.045364 & \text { vapor } \\ 2680.5 & 2903.2 & 6.4531 & 1.8751 & 2.6978 & 536.03 & 15.837 & 1.8137 \mathrm{e}-05 & 0.045424 & \text { vapor }\end{array}$ $\begin{array}{llllllllll}2680.5 & 2903.2 & 6.4531 & 1.8751 & 2.6978 & 536.03 & 15.837 & 1.8137 e-05 & 0.045424 & \text { vapor } \\ 2683.9 & 2907.7 & 6.4615 & 1.8670 & 2.6814 & 537.30 & 15.671 & 1.8213 e-05 & 0.045488 & \text { vapor }\end{array}$ $\begin{array}{lllllllllll}2687.3 & 2912.1 & 6.4698 & 1.8593 & 2.6656 & 538.57 & 15.507 & 1.8288 \mathrm{e}-05 & 0.045556 & \text { vapor }\end{array}$ $\begin{array}{llllllllll}2690.7 & 2916.6 & 6.4781 & 1.8519 & 2.6504 & 539.82 & 15.345 & 1.8364 \mathrm{e}-05 & 0.045626 & \text { vapor }\end{array}$ $\begin{array}{llllllllll}2694.1 & 2920.9 & 6.4862 & 1.8448 & 2.6359 & 541.06 & 15.186 & 1.8440 \mathrm{e}-05 & 0.045699 & \text { vapor }\end{array}$ $\begin{array}{llllllllll}2697.5 & 2925.3 & 6.4943 & 1.8380 & 2.6219 & 542.30 & 15.030 & 1.8515 \mathrm{e}-05 & 0.045775 & \text { vapor }\end{array}$ $\begin{array}{llllllllll}2700.8 & 2929.6 & 6.5024 & 1.8315 & 2.6084 & 543.52 & 14.875 & 1.8590 \mathrm{e}-05 & 0.045854 & \text { vapor }\end{array}$ $\begin{array}{llllllllll}2704.1 & 2934.0 & 6.5103 & 1.8252 & 2.5955 & 544.73 & 14.723 & 1.8666 \mathrm{e}-05 & 0.045935 & \text { vapor }\end{array}$ $\begin{array}{llllllllll}2707.4 & 2938.3 & 6.5182 & 1.8192 & 2.5830 & 545.93 & 14.573 & 1.8741 \mathrm{e}-05 & 0.046019 & \text { vapor }\end{array}$ $\begin{array}{llllllllll}2710.7 & 2942.5 & 6.5261 & 1.8135 & 2.5710 & 547.12 & 14.425 & 1.8816 \mathrm{e}-05 & 0.046106 & \text { vapor }\end{array}$

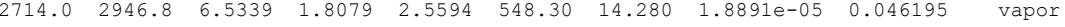
$\begin{array}{llllllllll}2717.3 & 2951.0 & 6.5416 & 1.8026 & 2.5482 & 549.47 & 14.136 & 1.8966 \mathrm{e}-05 & 0.046287 & \text { vapor }\end{array}$ $\begin{array}{llllllllll}2720.5 & 2955.3 & 6.5492 & 1.7974 & 2.5374 & 550.63 & 13.994 & 1.9041 \mathrm{e}-05 & 0.046380 & \text { vapor } \\ 2723.7 & 2959.5 & 6.5569 & 1.7925 & 2.5270 & 551.79 & 13.855 & 1.9115 \mathrm{e}-05 & 0.046477 & \text { vapor }\end{array}$ $\begin{array}{llllllllll}2726.9 & 2963.6 & 6.5644 & 1.7877 & 2.5170 & 552.93 & 13.717 & 1.9190 e-05 & 0.046575\end{array}$ $\begin{array}{llllllllll}2730.1 & 2967.8 & 6.5719 & 1.7832 & 2.5073 & 554.07 & 13.582 & 1.9265 e-05 & 0.046676 & \text { vapor }\end{array}$ $\begin{array}{llllllllll}2733.3 & 2972.0 & 6.5794 & 1.7788 & 2.4979 & 555.20 & 13.448 & 1.9339 \mathrm{e}-05 & 0.046778 & \text { vapor }\end{array}$ $\begin{array}{llllllllll}2736.5 & 2976.1 & 6.5868 & 1.7745 & 2.4888 & 556.32 & 13.317 & 1.9414 \mathrm{e}-05 & 0.046883 & \text { vapor }\end{array}$ $\begin{array}{llllllllll}2739.7 & 2980.2 & 6.5942 & 1.7704 & 2.4801 & 557.44 & 13.187 & 1.9488 \mathrm{e}-05 & 0.046990 & \text { vapor }\end{array}$ $\begin{array}{llllllllll}2742.8 & 2984.3 & 6.6015 & 1.7665 & 2.4716 & 558.55 & 13.059 & 1.9563 e-05 & 0.047099 & \text { vapor }\end{array}$ $\begin{array}{llllllllll}2746.0 & 2988.4 & 6.6087 & 1.7627 & 2.4634 & 559.65 & 12.932 & 1.9637 e-05 & 0.047209 & \text { vapor }\end{array}$ $\begin{array}{llllllllll}2749.1 & 2992.5 & 6.6160 & 1.7591 & 2.4555 & 560.74 & 12.808 & 1.9711 \mathrm{e}-05 & 0.047322 & \text { vapor }\end{array}$ $\begin{array}{llllllllll}2752.3 & 2996.6 & 6.6231 & 1.7556 & 2.4478 & 561.83 & 12.685 & 1.9785 e-05 & 0.047436 & \text { vapor }\end{array}$ $\begin{array}{llllllllll}2755.4 & 3000.7 & 6.6303 & 1.7522 & 2.4404 & 562.91 & 12.564 & 1.9860 \mathrm{e}-05 & 0.047553 & \text { vapor } \\ 2758.5 & 3004.7 & 6.6374 & 1.7489 & 2.4332 & 563.98 & 12.445 & 1.9934 \mathrm{e}-05 & 0.047670 & \text { vapor }\end{array}$ $\begin{array}{llllllllll}2761.6 & 3008.7 & 6.6444 & 1.7458 & 2.4263 & 565.05 & 12.327 & 2.0008 \mathrm{e}-05 & 0.047790 & \text { vapor }\end{array}$ $\begin{array}{llllllllll}2764.7 & 3012.8 & 6.6514 & 1.7428 & 2.4195 & 566.11 & 12.211 & 2.0082 \mathrm{e}-05 & 0.047911 & \text { vapor }\end{array}$ $\begin{array}{llllllllll}2767.8 & 3016.8 & 6.6584 & 1.7399 & 2.4130 & 567.17 & 12.097 & 2.0155 e-05 & 0.048034 & \text { vapor }\end{array}$ 


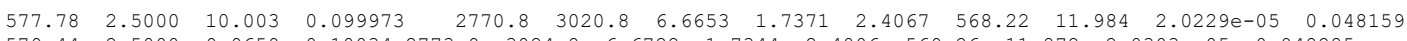
$\begin{array}{lllllllllllll}579.44 & 2.5000 & 9.9659 & 0.10034 & 2773.9 & 3024.8 & 6.6722 & 1.7344 & 2.4006 & 569.26 & 11.872 & 2.0303 e-05 & 0.048285\end{array}$ $\begin{array}{lllllllllllll}581.10 & 2.5000 & 9.9294 & 0.10071 & 2777.0 & 3028.7 & 6.6791 & 1.7318 & 2.3946 & 570.30 & 11.763 & 2.0377 \mathrm{e}-05 & 0.048412\end{array}$ $\begin{array}{lllllllllllll}582.76 & 2.5000 & 9.8933 & 0.10108 & 2780.0 & 3032.7 & 6.6859 & 1.7293 & 2.3889 & 571.33 & 11.654 & 2.0450 \mathrm{e}-05 & 0.048541\end{array}$ $\begin{array}{llllllllllllll}584.42 & 2.5000 & 9.8575 & 0.10145 & 2783.1 & 3036.7 & 6.6927 & 1.7269 & 2.3833 & 572.36 & 11.547 & 2.0524 \mathrm{e}-05 & 0.048672 & \text { vapor }\end{array}$ $\begin{array}{lllllllllllll}586.08 & 2.5000 & 9.8221 & 0.10181 & 2786.1 & 3040.6 & 6.6994 & 1.7246 & 2.3779 & 573.38 & 11.442 & 2.0598 \mathrm{e}-05 & 0.048804\end{array}$ $\begin{array}{llllllllllllll}587.74 & 2.5000 & 9.7870 & 0.10218 & 2789.1 & 3044.6 & 6.7062 & 1.7224 & 2.3727 & 574.40 & 11.338 & 2.0671 \mathrm{e}-05 & 0.048937 & \end{array}$

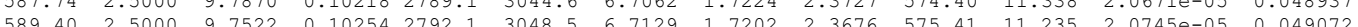

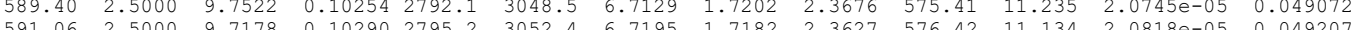
$\begin{array}{llllllllllllll}591.06 & 2.5000 & 9.7178 & 0.10290 & 2795.2 & 3052.4 & 6.7195 & 1.7182 & 2.3627 & 576.42 & 11.134 & 2.0818 \mathrm{e}-05 & 0.049207 & \text { vapor }\end{array}$

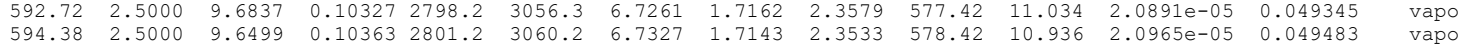
$\begin{array}{llllllllllllll}596.04 & 2.5000 & 9.6164 & 0.10399 & 2804.2 & 3064.2 & 6.7393 & 1.7125 & 2.3488 & 579.41 & 10.839 & 2.1038 \mathrm{e}-05 & 0.049623 & \text { vapor }\end{array}$

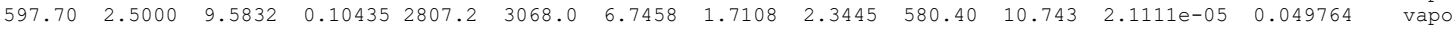
$\begin{array}{llllllllllllll}599.36 & 2.5000 & 9.5503 & 0.10471 & 2810.2 & 3071.9 & 6.7523 & 1.7091 & 2.3403 & 581.38 & 10.648 & 2.1184 \mathrm{e}-05 & 0.049906 & \text { vapor }\end{array}$

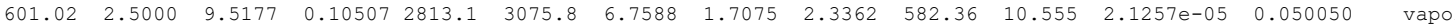
$\begin{array}{llllllllllllll}602.68 & 2.5000 & 9.4854 & 0.10543 & 2816.1 & 3079.7 & 6.7652 & 1.7059 & 2.3322 & 583.33 & 10.462 & 2.1330 \mathrm{e}-05 & 0.050194 & \text { vapor }\end{array}$ $\begin{array}{llllllllllllll}604.34 & 2.5000 & 9.4534 & 0.10578 & 2819.1 & 3083.6 & 6.7716 & 1.7045 & 2.3284 & 584.30 & 10.371 & 2.1403 \mathrm{e}-05 & 0.050340 & \text { vapor }\end{array}$ $\begin{array}{llllllllllllll}606.00 & 2.5000 & 9.4216 & 0.10614 & 2822.1 & 3087.4 & 6.7780 & 1.7030 & 2.3246 & 585.27 & 10.281 & 2.1476 \mathrm{e}-05 & 0.050487 & \text { vap }\end{array}$ $\begin{array}{llllllllllllll}607.66 & 2.5000 & 9.3901 & 0.10649 & 2825.0 & 3091.3 & 6.7843 & 1.7017 & 2.3210 & 586.23 & 10.193 & 2.1549 \mathrm{e}-05 & 0.050635 & \text { vapor }\end{array}$

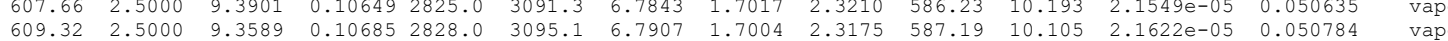

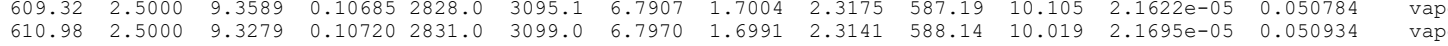

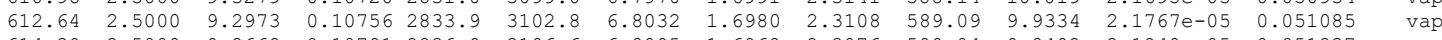
$\begin{array}{llllllllllllll}614.30 & 2.5000 & 9.2668 & 0.10791 & 2836.9 & 3106.6 & 6.8095 & 1.6968 & 2.3076 & 590.04 & 9.8492 & 2.1840 \mathrm{e}-05 & 0.051237 & \text { vapor }\end{array}$

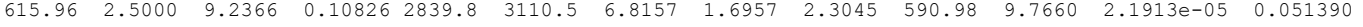

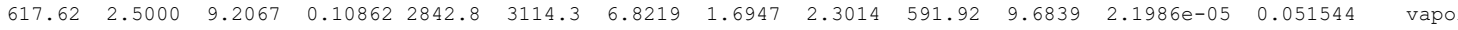

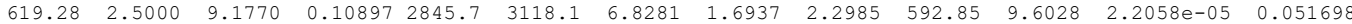
$\begin{array}{lllllllllllll}620.94 & 2.5000 & 9.1475 & 0.10932 & 2848.6 & 3121.9 & 6.8342 & 1.6928 & 2.2957 & 593.79 & 9.5227 & 2.2131 \mathrm{e}-05 & 0.051854\end{array}$

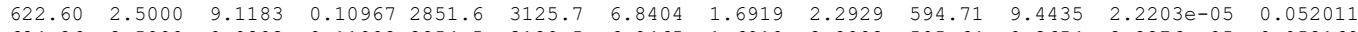
$\begin{array}{lllllllllllll}624.26 & 2.5000 & 9.0893 & 0.11002 & 2854.5 & 3129.5 & 6.8465 & 1.6910 & 2.2902 & 595.64 & 9.3654 & 2.2276 \mathrm{e}-05 & 0.052169\end{array}$ $\begin{array}{lllllllllllll}625.92 & 2.5000 & 9.0605 & 0.11037 & 2857.4 & 3133.3 & 6.8525 & 1.6902 & 2.2877 & 596.56 & 9.2883 & 2.2348 \mathrm{e}-05 & 0.052327\end{array}$

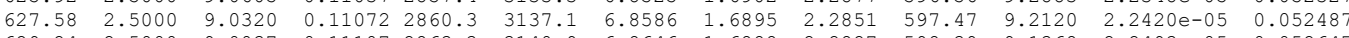

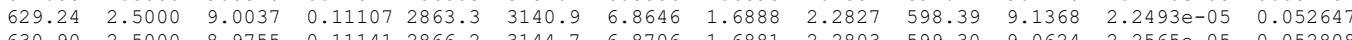
$\begin{array}{lllllllllllll}630.90 & 2.5000 & 8.9755 & 0.11141 & 2866.2 & 3144.7 & 6.8706 & 1.6881 & 2.2803 & 599.30 & 9.0624 & 2.2565 \mathrm{e}-05 & 0.052808\end{array}$

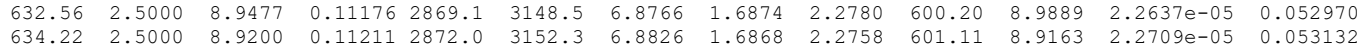

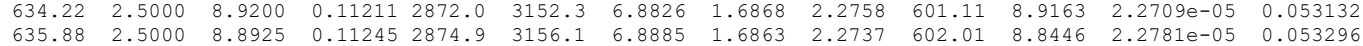
$\begin{array}{lllllllllllll}637.54 & 2.5000 & 8.8652 & 0.11280 & 2877.8 & 3159.8 & 6.8945 & 1.6857 & 2.2716 & 602.91 & 8.7738 & 2.2854 \mathrm{e}-05 & 0.053460\end{array}$ $\begin{array}{lllllllllllll}639.20 & 2.5000 & 8.8382 & 0.11315 & 2880.7 & 3163.6 & 6.9004 & 1.6852 & 2.2695 & 603.80 & 8.7037 & 2.2926 \mathrm{e}-05 & 0.053625\end{array}$

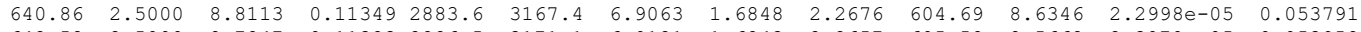
$\begin{array}{lllllllllllll}642.52 & 2.5000 & 8.7847 & 0.11383 & 2886.5 & 3171.1 & 6.9121 & 1.6843 & 2.2657 & 605.58 & 8.5662 & 2.3070 e-05 & 0.053958\end{array}$ $\begin{array}{lllllllllllll}644.18 & 2.5000 & 8.7582 & 0.11418 & 2889.4 & 3174.9 & 6.9180 & 1.6839 & 2.2638 & 606.47 & 8.4986 & 2.3142 \mathrm{e}-05 & 0.054125\end{array}$

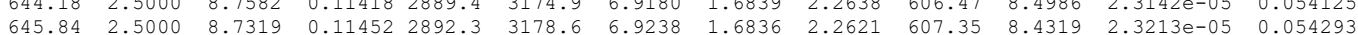

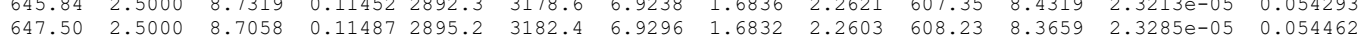
$\begin{array}{lllllllllllll}649.16 & 2.5000 & 8.6799 & 0.11521 & 2898.1 & 3186.1 & 6.9354 & 1.6829 & 2.2587 & 609.11 & 8.3006 & 2.3357 \mathrm{e}-05 & 0.054631\end{array}$

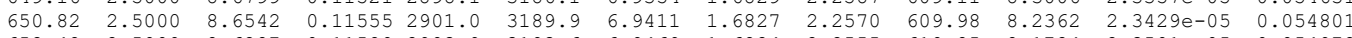
$\begin{array}{lllllllllllll}652.48 & 2.5000 & 8.6287 & 0.11589 & 2903.9 & 3193.6 & 6.9469 & 1.6824 & 2.2555 & 610.85 & 8.1724 & 2.3501 \mathrm{e}-05 & 0.054972\end{array}$

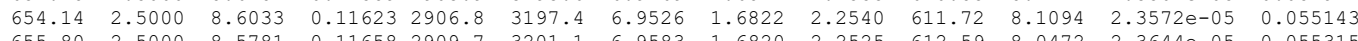

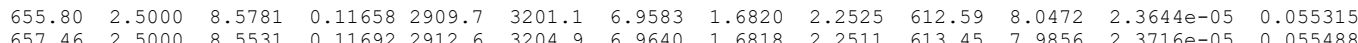
$\begin{array}{lllllllllllll}657.46 & 2.5000 & 8.5531 & 0.11692 & 2912.6 & 3204.9 & 6.9640 & 1.6818 & 2.2511 & 613.45 & 7.9856 & 2.3716 \mathrm{e}-05 & 0.055488 \\ 659.12 & 2.5000 & 8.5283 & 0.11726 & 2915.5 & 3208.6 & 6.9697 & 1.6817 & 2.2497 & 614.31 & 7.9247 & 2.3787 \mathrm{e}-05 & 0.055661\end{array}$

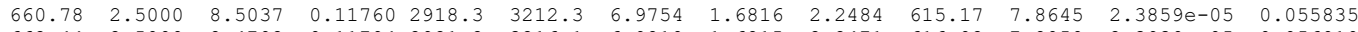
$\begin{array}{lllllllllllll}662.44 & 2.5000 & 8.4792 & 0.11794 & 2921.2 & 3216.1 & 6.9810 & 1.6815 & 2.2471 & 616.02 & 7.8050 & 2.3930 \mathrm{e}-05 & 0.056010\end{array}$ $\begin{array}{lllllllllllll}664.10 & 2.5000 & 8.4548 & 0.11828 & 2924.1 & 3219.8 & 6.9866 & 1.6814 & 2.2459 & 616.88 & 7.7461 & 2.4002 \mathrm{e}-05 & 0.056185\end{array}$

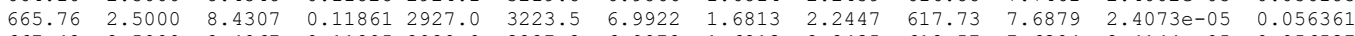
$\begin{array}{lllllllllllll}667.42 & 2.5000 & 8.4067 & 0.11895 & 2929.9 & 3227.2 & 6.9978 & 1.6813 & 2.2435 & 618.57 & 7.6304 & 2.4144 \mathrm{e}-05 & 0.056537\end{array}$ $\begin{array}{lllllllllllll}669.08 & 2.5000 & 8.3828 & 0.11929 & 2932.7 & 3231.0 & 7.0034 & 1.6813 & 2.2424 & 619.42 & 7.5734 & 2.4216 e-05 & 0.056714\end{array}$

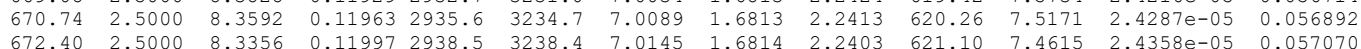

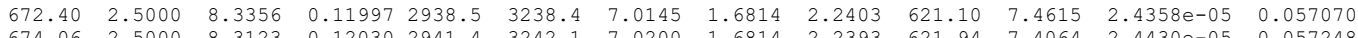
$\begin{array}{lllllllllllll}674.06 & 2.5000 & 8.3123 & 0.12030 & 2941.4 & 3242.1 & 7.0200 & 1.6814 & 2.2393 & 621.94 & 7.4064 & 2.4430 e-05 & 0.057248 \\ 675.72 & 2.5000 & 8.2891 & 0.12064 & 2944.2 & 3245.8 & 7.0255 & 1.6815 & 2.2383 & 622.78 & 7.3519 & 2.4501 \mathrm{e}-05 & 0.057428\end{array}$ $\begin{array}{lllllllllllll}677.38 & 2.5000 & 8.2660 & 0.12098 & 2947.1 & 3249.6 & 7.0310 & 1.6816 & 2.2374 & 623.61 & 7.2980 & 2.4572 \mathrm{e}-05 & 0.057607\end{array}$

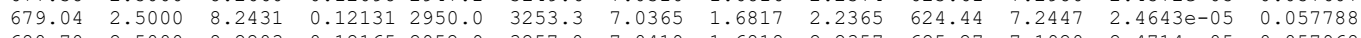
$\begin{array}{lllllllllllll}680.70 & 2.5000 & 8.2203 & 0.12165 & 2952.9 & 3257.0 & 7.0419 & 1.6818 & 2.2357 & 625.27 & 7.1920 & 2.4714 \mathrm{e}-05 & 0.057968\end{array}$

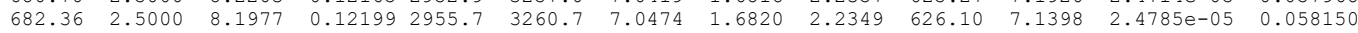
$\begin{array}{lllllllllllll}684.02 & 2.5000 & 8.1752 & 0.12232 & 2958.6 & 3264.4 & 7.0528 & 1.6822 & 2.2341 & 626.92 & 7.0882 & 2.4856 \mathrm{e}-05 & 0.058331\end{array}$ $\begin{array}{lllllllllllll}685.68 & 2.5000 & 8.1529 & 0.12266 & 2961.5 & 3268.1 & 7.0582 & 1.6823 & 2.2333 & 627.75 & 7.0371 & 2.4927 e-05 & 0.058514\end{array}$

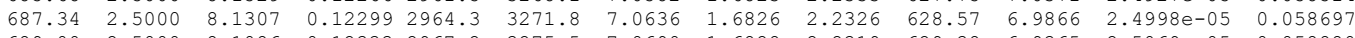

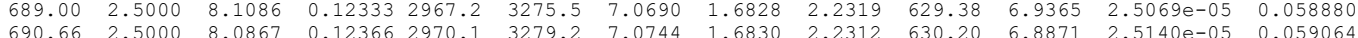
$\begin{array}{lllllllllllll}690.66 & 2.5000 & 8.0867 & 0.12366 & 2970.1 & 3279.2 & 7.0744 & 1.6830 & 2.2312 & 630.20 & 6.8871 & 2.5140 e-05 & 0.059064 \\ 6\end{array}$ $\begin{array}{lllllllllllll}692.32 & 2.5000 & 8.0649 & 0.12399 & 2972.9 & 3282.9 & 7.0797 & 1.6833 & 2.2306 & 631.01 & 6.8381 & 2.5210 \mathrm{e}-05 & 0.059248 \\ 693.98 & 2.5000 & 8.0433 & 0.12433 & 2975.8 & 3286.6 & 7.0851 & 1.6835 & 2.2300 & 631.83 & 6.7896 & 2.5281 e-05 & 0.059433\end{array}$

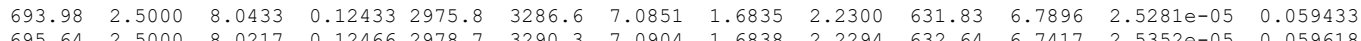
$\begin{array}{lllllllllllll}695.64 & 2.5000 & 8.0217 & 0.12466 & 2978.7 & 3290.3 & 7.0904 & 1.6838 & 2.2294 & 632.64 & 6.7417 & 2.5352 e-05 & 0.059618 \\ 697.30 & 2.5000 & 8.0004 & 0.12499 & 2981.5 & 3294.0 & 7.0957 & 1.6841 & 2.2289 & 633.44 & 6.6942 & 2.5423 e-05 & 0.059804\end{array}$ $\begin{array}{lllllllllllll}698.96 & 2.5000 & 7.9791 & 0.12533 & 2984.4 & 3297.7 & 7.1010 & 1.6844 & 2.2284 & 634.25 & 6.6472 & 2.5493 e-05 & 0.059990\end{array}$

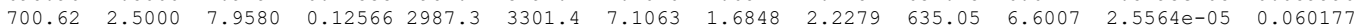

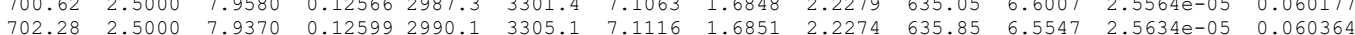

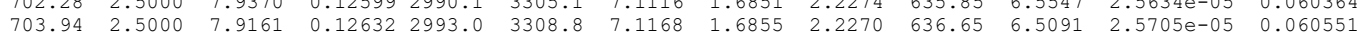

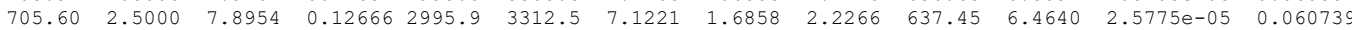
$\begin{array}{lllllllllllll}707.26 & 2.5000 & 7.8747 & 0.12699 & 2998.7 & 3316.2 & 7.1273 & 1.6862 & 2.2262 & 638.25 & 6.4193 & 2.5846 e-05 & 0.060928\end{array}$

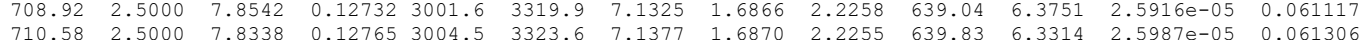

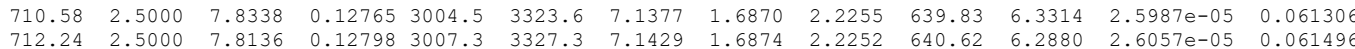
$\begin{array}{lllllllllllll}713.90 & 2.5000 & 7.7934 & 0.12831 & 3010.2 & 3331.0 & 7.1481 & 1.6879 & 2.2249 & 641.41 & 6.2451 & 2.6127 e-05 & 0.061686\end{array}$

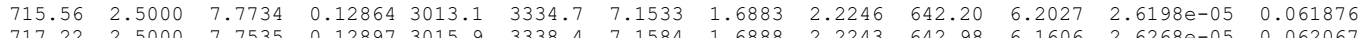

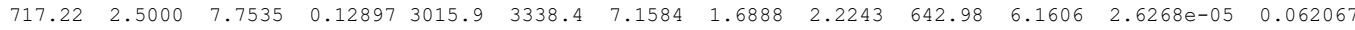

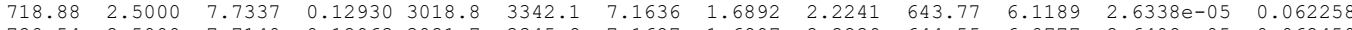
$\begin{array}{lllllllllllll}720.54 & 2.5000 & 7.7140 & 0.12963 & 3021.7 & 3345.8 & 7.1687 & 1.6897 & 2.2239 & 644.55 & 6.0777 & 2.6408 \mathrm{e}-05 & 0.062450\end{array}$

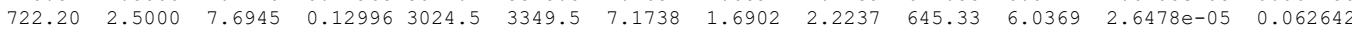
$\begin{array}{lllllllllllll}723.86 & 2.5000 & 7.6750 & 0.13029 & 3027.4 & 3353.1 & 7.1789 & 1.6907 & 2.2235 & 646.11 & 5.9964 & 2.6549 \mathrm{e}-05 & 0.062835\end{array}$ $\begin{array}{lllllllllllll}725.52 & 2.5000 & 7.6557 & 0.13062 & 3030.3 & 3356.8 & 7.1840 & 1.6912 & 2.2234 & 646.88 & 5.9563 & 2.6619 \mathrm{e}-05 & 0.063028\end{array}$ $\begin{array}{lllllllllllll}727.18 & 2.5000 & 7.6364 & 0.13095 & 3033.1 & 3360.5 & 7.1891 & 1.6917 & 2.2232 & 647.66 & 5.9167 & 2.6689 \mathrm{e}-05 & 0.063221 \\ 728.84 & 2.5000 & 7.6173 & 0.13128 & 3036.0 & 3364.2 & 7.1942 & 1.6922 & 2.2231 & 648.43 & 5.8774 & 2.6759 e-05 & 0.063415\end{array}$ $\begin{array}{llllllllllllll}728.84 & 2.5000 & 7.6173 & 0.13128 & 3036.0 & 3364.2 & 7.1942 & 1.6922 & 2.2231 & 648.43 & 5.8774 & 2.6759 \mathrm{e}-05 & 0.063415 \\ 7330.50 & 2.5000 & 7.5983 & 0.13161 & 3038.9 & 3367.9 & 7.1992 & 1.6928 & 2.2230 & 649.20 & 5.8384 & 2.6829 e-05 & 0.063609\end{array}$

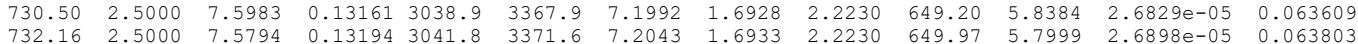

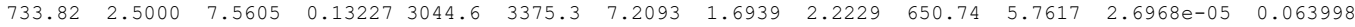




\begin{tabular}{|c|c|c|c|}
\hline 735.48 & .5000 & .5418 & 0.13259 \\
\hline 737.14 & 2.5000 & 7.5232 & 0.13292 \\
\hline 738.80 & 2.5000 & 7.5047 & 0.13325 \\
\hline 740.46 & 2.5000 & 7.4863 & 0.13358 \\
\hline 742.12 & 2.5000 & 7.4680 & 0.13390 \\
\hline 43.78 & .5000 & 7.4498 & 0.13423 \\
\hline 745.44 & 2.5000 & 7.4317 & 0.13456 \\
\hline 747.10 & 2.5000 & 7.4137 & 0.13489 \\
\hline 748.76 & 2.5000 & 7.3958 & 0.13521 \\
\hline 750.42 & 2.5000 & 7.3780 & 0.13554 \\
\hline 752.08 & 2.5000 & 7.3603 & 0.13586 \\
\hline 753.74 & 2.5000 & 7.3426 & 0.13619 \\
\hline 755.40 & 2.5000 & 7.3251 & 0.13652 \\
\hline 757.06 & 2.5000 & 7.3077 & 0.13684 \\
\hline 758.72 & 2.5000 & 7.2903 & 0.13717 \\
\hline 760.38 & 2.5000 & 7.2731 & 0.13749 \\
\hline 762.04 & 2.5000 & 7.2559 & 0.13782 \\
\hline 763.70 & 2.5000 & 7.2388 & 0.13814 \\
\hline 765.36 & 2.5000 & 7.2218 & 0.13847 \\
\hline 767.02 & 2.5000 & 7.2049 & 0.13879 \\
\hline 768.68 & 2.5000 & 7.1881 & 0.13912 \\
\hline 770.34 & 2.5000 & 7.1714 & 0.13944 \\
\hline 772.00 & 2.5000 & 7.1547 & 0.13977 \\
\hline 773.66 & 2.5000 & 7.1382 & 0.14009 \\
\hline 775.32 & 2.5000 & 7.1217 & 0.14042 \\
\hline 776.98 & 2.5000 & 7.1053 & 0.14074 \\
\hline 778.64 & 2.5000 & 7.0890 & 0.14106 \\
\hline 780.30 & 2.5000 & 7.0728 & 0.14139 \\
\hline 781.96 & 2.5000 & 7.0566 & 0.14171 \\
\hline 783.62 & 2.5000 & 7.0405 & 0.14203 \\
\hline 785.28 & 2.5000 & 7.0246 & 0.14236 \\
\hline 786.94 & 2.5000 & 7.0086 & 0.14268 \\
\hline 788.60 & 2.5000 & 6.9928 & 0.14300 \\
\hline 790.26 & 2.5000 & 6.9771 & 0.14333 \\
\hline 791.92 & 2.5000 & 6.9614 & 0.14365 \\
\hline 793.58 & 2.5000 & 6.9458 & 0.14397 \\
\hline 795.24 & 2.5000 & 6.9303 & 0.14429 \\
\hline 796.90 & 2.5000 & 6.9148 & 0.14462 \\
\hline 798.56 & 2.5000 & 6.8994 & 0.14494 \\
\hline 800.22 & 2.5000 & 6.8841 & 0.14526 \\
\hline 801.88 & 2.5000 & 6.8689 & 0.14558 \\
\hline 803.54 & 2.5000 & 6.8537 & 0.14591 \\
\hline 805.20 & 2.5000 & 6.8387 & 0.14623 \\
\hline 806.86 & 2.5000 & 6.8236 & 0.14655 \\
\hline 808.52 & 2.5000 & 6.8087 & 0.14687 \\
\hline 810.18 & 2.5000 & 6.7938 & 0.14719 \\
\hline 811.84 & 2.5000 & 6.7790 & 0.14751 \\
\hline 813.50 & 2.5000 & 6.7643 & 0.14783 \\
\hline 815.16 & 2.5000 & 6.7496 & 0.14816 \\
\hline 816.82 & 2.5000 & 6.7350 & 0.14848 \\
\hline 818.48 & 2.5000 & 6.7205 & 0.14880 \\
\hline 820.14 & 2.5000 & 6.7061 & 0.14912 \\
\hline 821.80 & 2.5000 & 6.6917 & 0.14944 \\
\hline 823.46 & 2.5000 & 6.6773 & 0.14976 \\
\hline 825.12 & 2.5000 & 6.6631 & 0.15008 \\
\hline 826.78 & 2.5000 & 6.6489 & 0.15040 \\
\hline 828.44 & 2.5000 & 6.6348 & 0.15072 \\
\hline 830.10 & 2.5000 & 6.6207 & 0.15104 \\
\hline 831.76 & 2.5000 & 6.6067 & 0.15136 \\
\hline 833.42 & 2.5000 & 6.5928 & 0.15168 \\
\hline 835.08 & 2.5000 & 6.5789 & 0.15200 \\
\hline 836.74 & 2.5000 & 6.5651 & 0.15232 \\
\hline 838.40 & 2.5000 & 6.5513 & 0.15264 \\
\hline 840.06 & 2.5000 & 6.5376 & 0.15296 \\
\hline 841.72 & 2.5000 & 6.5240 & 0.15328 \\
\hline 843.38 & 2.5000 & 6.5104 & 0.15360 \\
\hline 845.04 & 2.5000 & 6.4969 & 0.15392 \\
\hline 846.70 & 2.5000 & 6.4835 & 0.15424 \\
\hline 848.36 & 2.5000 & 6.4701 & 0.15456 \\
\hline 850.02 & 2.5000 & 6.4567 & 0.15488 \\
\hline 851.68 & 2.5000 & 6.4435 & 0.15520 \\
\hline 853.34 & 2.5000 & 6.4303 & 0.15551 \\
\hline 855.00 & 2.5000 & 6.4171 & 0.15583 \\
\hline 856.66 & 2.5000 & 6.4040 & 0.15615 \\
\hline 858.32 & 2.5000 & 6.3910 & 0.15647 \\
\hline 859.98 & 2.5000 & 6.3780 & 0.15679 \\
\hline 861.64 & 2.5000 & 6.3651 & 0.15711 \\
\hline 863.30 & 2.5000 & 6.3522 & 0.15743 \\
\hline 864.96 & 2.5000 & 6.3394 & 0.15774 \\
\hline 866.62 & 2.5000 & 6.3266 & 0.15806 \\
\hline 868.28 & 2.5000 & 6.3139 & 0.15838 \\
\hline 869.94 & 2.5000 & 6.3012 & 0.15870 \\
\hline 871.60 & 2.5000 & 6.2886 & 0.15902 \\
\hline 873.26 & 2.5000 & 6.2761 & 0.15934 \\
\hline 874.92 & 2.5000 & 6.2636 & 0.15965 \\
\hline 876.58 & 2.5000 & 6.2511 & 0.15997 \\
\hline 878.24 & 2.5000 & 6.2388 & 0.16029 \\
\hline 879.90 & 2.5000 & 6.2264 & 0.16061 \\
\hline 881.56 & 2.5000 & 6.2141 & 0.16092 \\
\hline 883.22 & 2.5000 & 6.2019 & 0.16124 \\
\hline 884.88 & 2.5000 & 6.1897 & 0.16156 \\
\hline 886.54 & 2.5000 & 6.1776 & 0.16188 \\
\hline 888.20 & 2.5000 & 6.1655 & 0.16219 \\
\hline 889.86 & 2.5000 & 6.1535 & 5251 \\
\hline 891 & 2.5000 & 6.1415 & 0.16283 \\
\hline
\end{tabular}




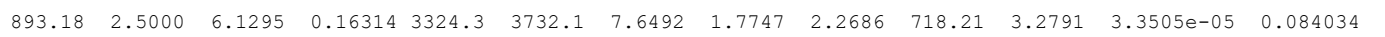
$\begin{array}{lllllllllllllll}896.50 & 2.5000 & 6.1058 & 0.16378 & 3330.2 & 3739.7 & 7.6576 & 1.7767 & 2.2694 & 718.86 & 3.2618 & 3.3571 \mathrm{e}-05 & 0.084253 & \text { vapor }\end{array}$

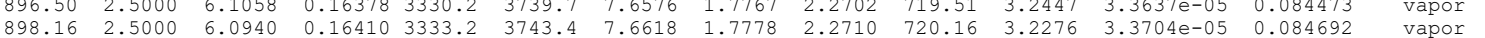
$\begin{array}{llllllllllllll}899.82 & 2.5000 & 6.0823 & 0.16441 & 3336.2 & 3747.2 & 7.6660 & 1.7788 & 2.2719 & 720.80 & 3.2107 & 3.3770 \mathrm{e}-05 & 0.084912 & \text { vapor }\end{array}$

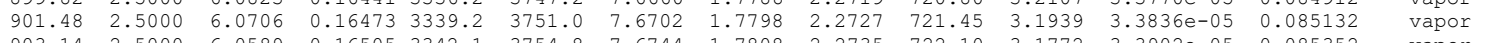
$\begin{array}{llllllllllllll}903.14 & 2.5000 & 6.0589 & 0.16505 & 3342.1 & 3754.8 & 7.6744 & 1.7808 & 2.2735 & 722.10 & 3.1772 & 3.3902 \mathrm{e}-05 & 0.085352 & \text { vapor }\end{array}$ $\begin{array}{llllllllllllll}904.80 & 2.5000 & 6.0473 & 0.16536 & 3345.1 & 3758.5 & 7.6786 & 1.7818 & 2.2743 & 722.74 & 3.1606 & 3.3968 \mathrm{e}-05 & 0.085572 & \text { vapor } \\ 906.46 & 2.5000 & 6.0358 & 0.16568 & 3348.1 & 3762.3 & 7.6827 & 1.7829 & 2.2751 & 723.38 & 3.1442 & 3.4034 \mathrm{e}-05 & 0.085793 & \text { vapor }\end{array}$ $\begin{array}{llllllllllllll}906.46 & 2.5000 & 6.0358 & 0.16568 & 3348.1 & 3762.3 & 7.6827 & 1.7829 & 2.2751 & 723.38 & 3.1442 & 3.4034 \mathrm{e}-05 & 0.085793 & \text { vapor } \\ 908.12 & 2.5000 & 6.0243 & 0.16600 & 3351.1 & 3766.1 & 7.6869 & 1.7839 & 2.2760 & 724.03 & 3.1279 & 3.4100 \mathrm{e}-05 & 0.086013 & \text { vapor }\end{array}$ $\begin{array}{llllllllllllll}909.78 & 2.5000 & 6.0128 & 0.16631 & 3354.1 & 3769.9 & 7.6911 & 1.7849 & 2.2768 & 724.67 & 3.1116 & 3.4166 \mathrm{e}-05 & 0.086234 & \text { vapor }\end{array}$ $\begin{array}{llllllllllllll}911.44 & 2.5000 & 6.0014 & 0.16663 & 3357.1 & 3773.6 & 7.6952 & 1.7860 & 2.2777 & 725.31 & 3.0955 & 3.4232 e-05 & 0.086455 & \text { vapor }\end{array}$

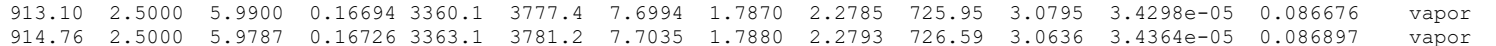
$\begin{array}{lllllllllllllll}916.42 & 2.5000 & 5.9674 & 0.16758 & 3366.1 & 3785.0 & 7.7076 & 1.7891 & 2.2802 & 727.23 & 3.0479 & 3.4430 \mathrm{e}-05 & 0.087118 & \text { vapor }\end{array}$

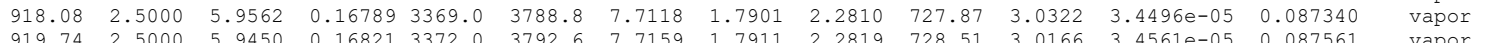

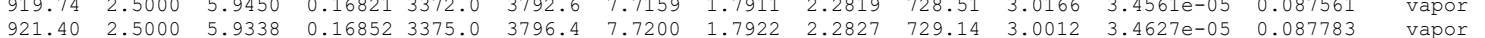

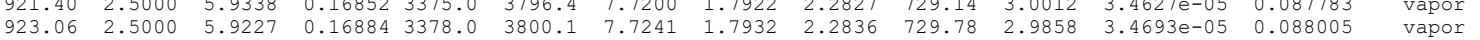

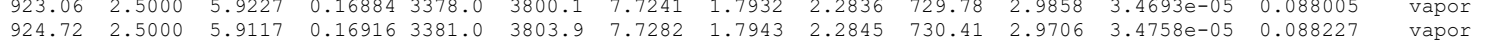

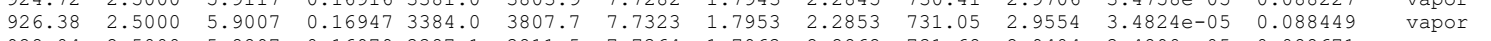

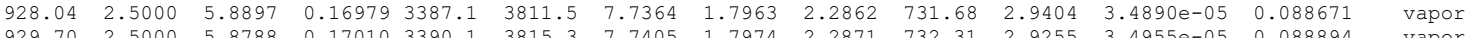

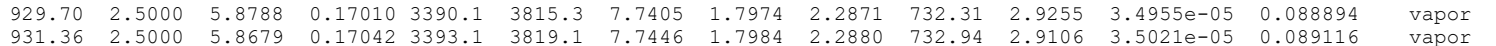
$\begin{array}{llllllllllllll}933.02 & 2.5000 & 5.8570 & 0.17074 & 3396.1 & 3822.9 & 7.7486 & 1.7995 & 2.2888 & 733.58 & 2.8959 & 3.5086 \mathrm{e}-05 & 0.089339 & \text { vapor }\end{array}$

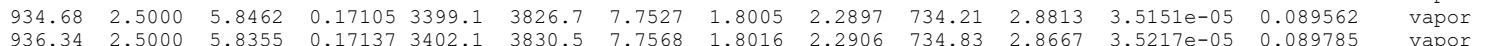

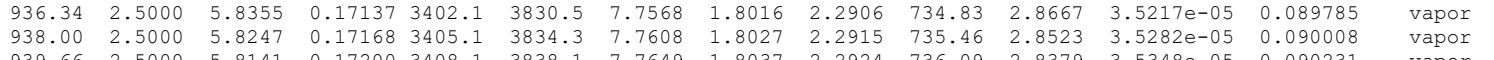
$\begin{array}{llllllllllllll}939.66 & 2.5000 & 5.8141 & 0.17200 & 3408.1 & 3838.1 & 7.7649 & 1.8037 & 2.2924 & 736.09 & 2.8379 & 3.5348 \mathrm{e}-05 & 0.090231 & \text { vapor }\end{array}$

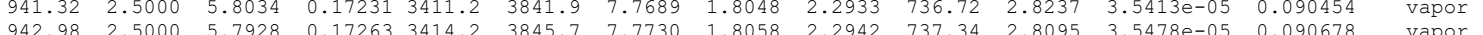
$\begin{array}{llllllllllllll}942.98 & 2.5000 & 5.7928 & 0.17263 & 3414.2 & 3845.7 & 7.7730 & 1.8058 & 2.2942 & 737.34 & 2.8095 & 3.5478 \mathrm{e}-05 & 0.090678 & \text { vapor } \\ 944.64 & 2.5000 & 5.7823 & 0.17294 & 3417.2 & 3849.5 & 7.7770 & 1.8069 & 2.2951 & 737.97 & 2.7955 & 3.5543 e-05 & 0.090902 & \text { vapor }\end{array}$ $\begin{array}{llllllllllllll}946.30 & 2.5000 & 5.7717 & 0.17326 & 3420.2 & 3853.4 & 7.7810 & 1.8079 & 2.2960 & 738.59 & 2.7815 & 3.5608 \mathrm{e}-05 & 0.091125 & \text { vapor }\end{array}$

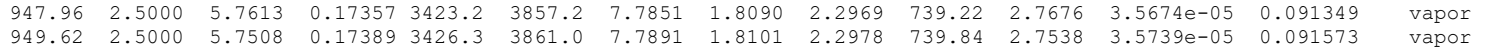
$\begin{array}{llllllllllllll}951.28 & 2.5000 & 5.7404 & 0.17420 & 3429.3 & 3864.8 & 7.7931 & 1.8111 & 2.2987 & 740.46 & 2.7402 & 3.5804 \mathrm{e}-05 & 0.091797 & \text { vapor }\end{array}$ $\begin{array}{llllllllllllll}952.94 & 2.5000 & 5.7301 & 0.17452 & 3432.3 & 3868.6 & 7.7971 & 1.8122 & 2.2996 & 741.08 & 2.7265 & 3.5869 \mathrm{e}-05 & 0.092022 & \text { vapor } \\ 954.60 & 2.5000 & 5.7197 & 0.17483 & 3435.4 & 3872.4 & 7.8011 & 1.8133 & 2.3005 & 741.70 & 2.7130 & 3.5934 \mathrm{e}-05 & 0.092246 & \text { vapor }\end{array}$

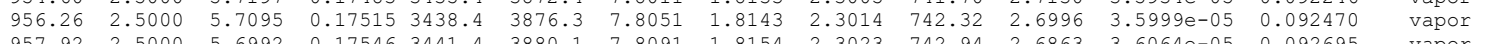
$\begin{array}{llllllllllllll}957.92 & 2.5000 & 5.6992 & 0.17546 & 3441.4 & 3880.1 & 7.8091 & 1.8154 & 2.3023 & 742.94 & 2.6863 & 3.6064 \mathrm{e}-05 & 0.092695 & \text { vapor }\end{array}$

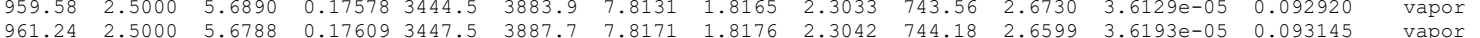

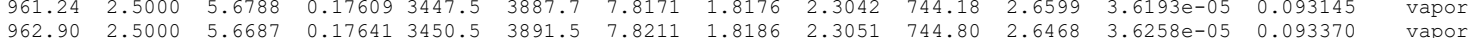
$\begin{array}{llllllllllllll}962.90 & 2.5000 & 5.6687 & 0.17641 & 3450.5 & 3891.5 & 7.8211 & 1.8186 & 2.3051 & 744.80 & 2.6468 & 3.6258 \mathrm{e}-05 & 0.093370 & \text { vapor } \\ 964.56 & 2.5000 & 5.6586 & 0.17672 & 3453.6 & 3895.4 & 7.8250 & 1.8197 & 2.3060 & 745.41 & 2.6338 & 3.6323 e-05 & 0.093595 & \text { vapor }\end{array}$

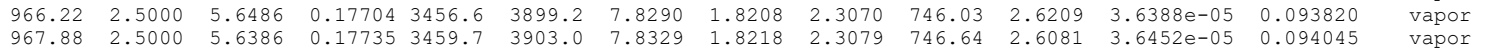

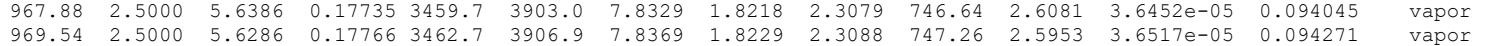

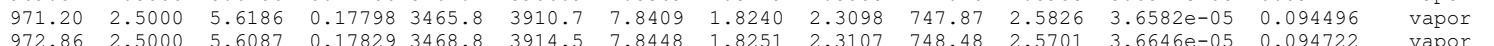

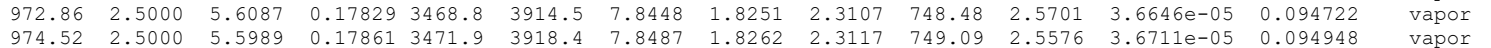

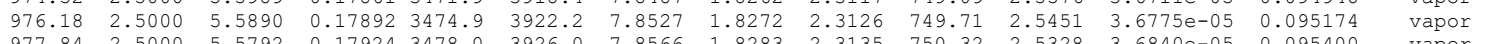

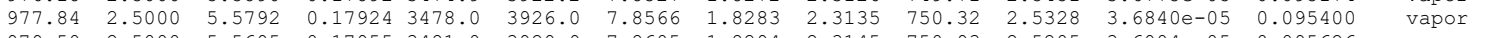

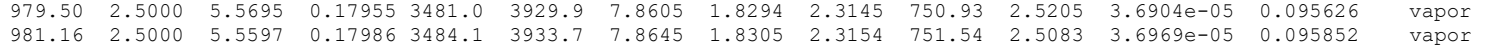

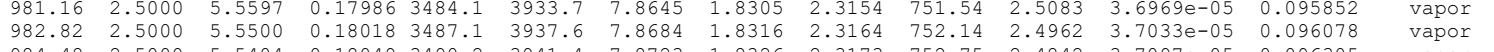

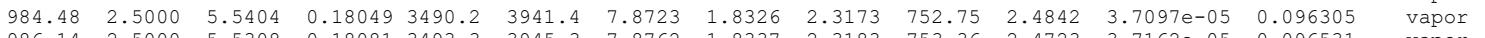

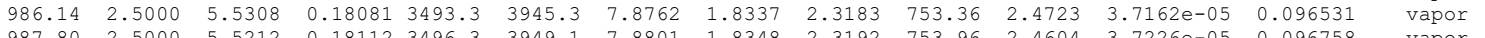
$\begin{array}{llllllllllllll}987.80 & 2.5000 & 5.5212 & 0.18112 & 3496.3 & 3949.1 & 7.8801 & 1.8348 & 2.3192 & 753.96 & 2.4604 & 3.7226 \mathrm{e}-05 & 0.096758 & \text { vapor } \\ 989.46 & 2.5000 & 5.5116 & 0.18144 & 3499.4 & 3953.0 & 7.8840 & 1.8359 & 2.3202 & 754.57 & 2.4486 & 3.7290 \mathrm{e}-05 & 0.096985 & \text { vapor }\end{array}$ $\begin{array}{llllllllllllll}991.12 & 2.5000 & 5.5021 & 0.18175 & 3502.5 & 3956.8 & 7.8879 & 1.8370 & 2.3212 & 755.17 & 2.4368 & 3.7354 \mathrm{e}-05 & 0.097212 & \text { vapor }\end{array}$ $\begin{array}{llllllllllllll}992.78 & 2.5000 & 5.4926 & 0.18206 & 3505.5 & 3960.7 & 7.8918 & 1.8381 & 2.3221 & 755.78 & 2.4252 & 3.7419 e-05 & 0.097439 & \text { vapor }\end{array}$

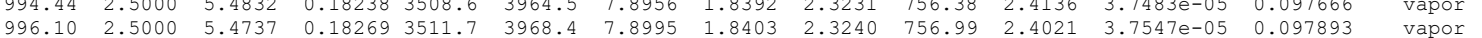

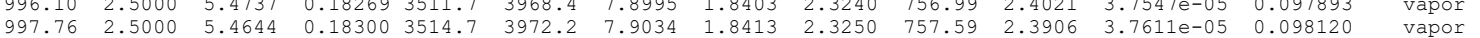

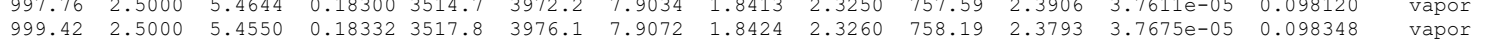
$\begin{array}{llllllllllllll}999.42 & 2.5000 & 5.4550 & 0.18332 & 3517.8 & 3976.1 & 7.9072 & 1.8424 & 2.3260 & 758.19 & 2.3793 & 3.7675 e-05 & 0.098348 & \text { vapor } \\ 1001.1 & 2.5000 & 5.4457 & 0.18363 & 3520.9 & 3980.0 & 7.9111 & 1.8435 & 2.3269 & 758.79 & 2.3679 & 3.7739 e-05 & 0.098575 & \text { vapor }\end{array}$

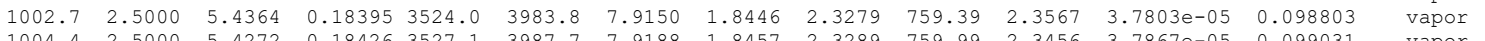

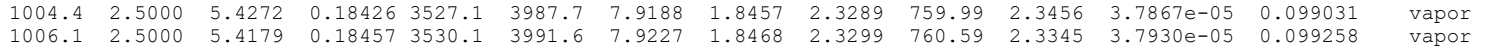
$\begin{array}{llllllllllllll}1007.7 & 2.5000 & 5.4087 & 0.18489 & 3533.2 & 3995.4 & 7.9265 & 1.8479 & 2.3308 & 761.19 & 2.3234 & 3.7994 \mathrm{e}-05 & 0.099486 & \text { vapor }\end{array}$

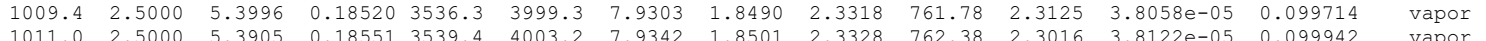
$\begin{array}{llllllllllllll}1012.7 & 2.5000 & 5.3814 & 0.18583 & 3542.5 & 4007.0 & 7.9380 & 1.8512 & 2.3338 & 762.98 & 2.2908 & 3.8186 \mathrm{e}-05 & 0.10017 \text { vapor }\end{array}$ $\begin{array}{lllllllllllll}1014.4 & 2.5000 & 5.3723 & 0.18614 & 3545.6 & 4010.9 & 7.9418 & 1.8523 & 2.3347 & 763.57 & 2.2800 & 3.8249 \mathrm{e}-05 & 0.10040 \text { vapor }\end{array}$ $\begin{array}{lllllllllllll}1016.0 & 2.5000 & 5.3633 & 0.18645 & 3548.7 & 4014.8 & 7.9456 & 1.8534 & 2.3357 & 764.17 & 2.2693 & 3.8313 e-05 & 0.10063 \\ 10 a p o r\end{array}$

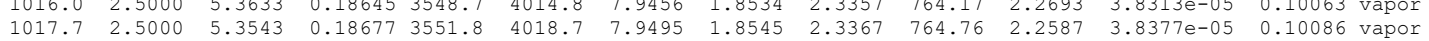
$\begin{array}{lllllllllllll}1019.3 & 2.5000 & 5.3453 & 0.18708 & 3554.9 & 4022.6 & 7.9533 & 1.8556 & 2.3377 & 765.36 & 2.2481 & 3.8440 \mathrm{e}-05 & 0.10108 \text { vapor }\end{array}$ $\begin{array}{lllllllllllll}1021.0 & 2.5000 & 5.3364 & 0.18739 & 3558.0 & 4026.4 & 7.9571 & 1.8567 & 2.3387 & 765.95 & 2.2376 & 3.8504 \mathrm{e}-05 & 0.10131 \\ 10 a p o r\end{array}$

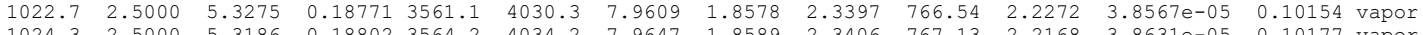

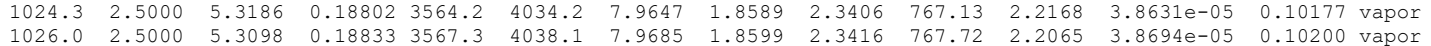
$\begin{array}{lllllllllllll}1027.6 & 2.5000 & 5.3010 & 0.18864 & 3570.4 & 4042.0 & 7.9722 & 1.8610 & 2.3426 & 768.31 & 2.1962 & 3.8757 e-05 & 0.10223 \\ \text { vapor }\end{array}$ $\begin{array}{lllllllllllll}1029.3 & 2.5000 & 5.2922 & 0.18896 & 3573.5 & 4045.9 & 7.9760 & 1.8621 & 2.3436 & 768.90 & 2.1860 & 3.8821 \mathrm{e}-05 & 0.10246 \text { vapor }\end{array}$ $\begin{array}{lllllllllllll}1031.0 & 2.5000 & 5.2834 & 0.18927 & 3576.6 & 4049.8 & 7.9798 & 1.8632 & 2.3446 & 769.49 & 2.1759 & 3.8884 \mathrm{e}-05 & 0.10269 \\ 1032.6 & 2.5000 & 5.2747 & 0.18958 & 3579.7 & 4053.7 & 7.9836 & 1.8643 & 2.3456 & 770.08 & 2.1658 & 3.8947\end{array}$

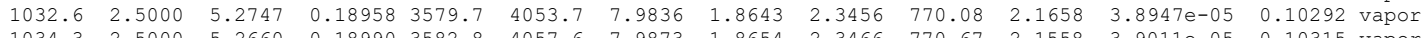
$\begin{array}{lllllllllllll}1034.3 & 2.5000 & 5.2660 & 0.18990 & 3582.8 & 4057.6 & 7.9873 & 1.8654 & 2.3466 & 770.67 & 2.1558 & 3.9011 \mathrm{e}-05 & 0.10315 \\ 103 a p o r\end{array}$ $\begin{array}{lllllllllllll}1035.9 & 2.5000 & 5.2574 & 0.19021 & 3585.9 & 4061.4 & 7.9911 & 1.8665 & 2.3476 & 771.26 & 2.1459 & 3.9074 \mathrm{e}-05 & 0.10338 \text { vapor } \\ 1037.6 & 2.5000 & 5.2487 & 0.19052 & 3589.0 & 4065.3 & 7.9949 & 1.8676 & 2.3486 & 771.84 & 2.1360 & 3.9137 \mathrm{e}-05 & 0.10361 \text { vapor }\end{array}$ $\begin{array}{lllllllllllll}1037.6 & 2.5000 & 5.2487 & 0.19052 & 3589.0 & 4065.3 & 7.9949 & 1.8676 & 2.3486 & 771.84 & 2.1360 & 3.9137 \mathrm{e}-05 & 0.10361 \text { vapor } \\ 1039.3 & 2.5000 & 5.2401 & 0.19084 & 3592.2 & 4069.2 & 7.9986 & 1.8687 & 2.3496 & 772.43 & 2.1262 & 3.9200 \mathrm{e}-05 & 0.10384 \text { vapor }\end{array}$ $\begin{array}{lllllllllllll}1039.3 & 2.5000 & 5.2401 & 0.19084 & 3592.2 & 4069.2 & 7.9986 & 1.8687 & 2.3496 & 772.43 & 2.1262 & 3.9200 e-05 & 0.10384 \\ 1040.9 & 2.5000 & 5.2315 & 0.19115 & 3595.3 & 4073.1 & 8.0024 & 1.8698 & 2.3506 & 773.02 & 2.1164 & 3.9263 \mathrm{e}-05 & 0.10407 \text { vapor }\end{array}$ $\begin{array}{llllllllllllll}1042.6 & 2.5000 & 5.2230 & 0.19146 & 3598.4 & 4077.0 & 8.0061 & 1.8709 & 2.3516 & 773.60 & 2.1067 & 3.9326 \mathrm{e}-05 & 0.10430 & \text { vapor }\end{array}$ $\begin{array}{lllllllllllll}1044.2 & 2.5000 & 5.2145 & 0.19177 & 3601.5 & 4081.0 & 8.0099 & 1.8720 & 2.3526 & 774.19 & 2.0970 & 3.9389 \mathrm{e}-05 & 0.10453 \\ \text { vapor }\end{array}$ $\begin{array}{lllllllllllll}1045.9 & 2.5000 & 5.2060 & 0.19209 & 3604.6 & 4084.9 & 8.0136 & 1.8732 & 2.3536 & 774.77 & 2.0874 & 3.9452 \mathrm{e}-05 & 0.10476 \text { vapor } \\ 1047.6 & 2.5000 & 5.1975 & 0.19240 & 3607.8 & 4088.8 & 8.0173 & 1.8743 & 2.3546 & 775.35 & 2.0779 & 3.9515 \mathrm{e}-05 & 0.10499 \text { vapor }\end{array}$

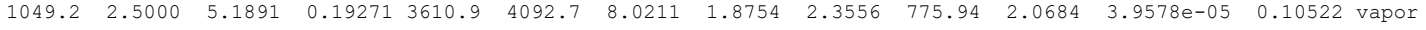




\begin{tabular}{|c|c|c|c|}
\hline 1050.9 & .5000 & .1807 & 0.19302 \\
\hline 1052.5 & .5000 & .1723 & 0.19334 \\
\hline 1054.2 & .5000 & .1640 & . 19365 \\
\hline 055.9 & .5000 & .1557 & 0.19396 \\
\hline 057.5 & .5000 & .1474 & .19427 \\
\hline 59.2 & .5000 & .1391 & . 19459 \\
\hline 60.8 & .5000 & 5.1309 & .19490 \\
\hline 62.5 & & & \\
\hline 064.2 & .5000 & .1145 & 0.19552 \\
\hline 065.8 & .5000 & 5.1063 & 0.19584 \\
\hline 067.5 & .5000 & .0982 & 0.19615 \\
\hline 069.1 & .5000 & 5.0901 & 0.19646 \\
\hline 070.8 & .5000 & .0820 & 0.19677 \\
\hline 072.5 & .5000 & 5.0740 & 0.19708 \\
\hline 074.1 & .5000 & .0659 & 0.19740 \\
\hline .075 .8 & .5000 & .0579 & 0.19771 \\
\hline 077.4 & .5000 & 5.0500 & 802 \\
\hline 079.1 & .5000 & .0420 & 0.19833 \\
\hline 080.8 & .5000 & 5.0341 & 0.19865 \\
\hline 1082.4 & .5000 & 5.0262 & 0.19896 \\
\hline 1084.1 & .5000 & .0183 & 0.19927 \\
\hline 1085.7 & 2.5000 & 5.0105 & 0.19958 \\
\hline 1087.4 & .5000 & 5.0027 & 0.19989 \\
\hline 1089.1 & .5000 & 4.9949 & 0.20021 \\
\hline 90.7 & .5000 & 4.9871 & 052 \\
\hline 092.4 & .5000 & .9794 & 0.20083 \\
\hline 1094.0 & .5000 & 4.9716 & 0.20114 \\
\hline 1095.7 & .5000 & 4.9639 & 0.20145 \\
\hline 97.4 & .5000 & 4.9563 & 176 \\
\hline 1099.0 & 2.5000 & 4.9486 & 208 \\
\hline 1100.7 & .5000 & 4.9410 & 0.20239 \\
\hline 02.3 & .5000 & 4.9334 & 270 \\
\hline 104.0 & .5000 & 4.9258 & 0.20301 \\
\hline 05.7 & .5000 & 4.9183 & 0.20332 \\
\hline 1107.3 & 2.5000 & 4.9108 & 0.20363 \\
\hline 1109.0 & 2.5000 & 4.9033 & 0.20395 \\
\hline 10.6 & .5000 & 4.8958 & 426 \\
\hline 1112.3 & 2.5000 & 4.8883 & 457 \\
\hline 1114.0 & .5000 & 4.8809 & 0.20488 \\
\hline 15.6 & .5000 & 4.8735 & 519 \\
\hline 17.3 & .5000 & 4.8661 & 550 \\
\hline 18.9 & .5000 & 4.8587 & 582 \\
\hline 1120.6 & 2.5000 & 4.8514 & 613 \\
\hline 1122.3 & .5000 & 4.8441 & 0.20644 \\
\hline 23.9 & .5000 & 4.8368 & 675 \\
\hline 5.6 & .5000 & 4.8 & 706 \\
\hline 27.2 & .5000 & 4.8223 & 737 \\
\hline 1128.9 & 2.5000 & 4.8150 & 0.20768 \\
\hline 1130.6 & .5000 & 4.8078 & 0.20799 \\
\hline 1132.2 & 2.5000 & 4.8006 & 0.20831 \\
\hline 1133.9 & 2.5000 & 4.7935 & 0.20862 \\
\hline 35.5 & .5000 & 4.7 & 893 \\
\hline 1137.2 & .5000 & 4.7792 & 0924 \\
\hline 1138.9 & .5000 & 4.7721 & 0.20955 \\
\hline 40.5 & 2.5000 & 4.7650 & 0.20986 \\
\hline 1142.2 & 2.5000 & 4.7580 & 0.21017 \\
\hline 1143.8 & .5000 & 4. & 048 \\
\hline 1145.5 & .5000 & 4.7439 & 0.21080 \\
\hline 7.2 & .5000 & 4. & 111 \\
\hline 8.8 & .5000 & 4.7300 & 142 \\
\hline 1150.5 & 2.5000 & 4.7230 & 0.21173 \\
\hline 1152.1 & .5000 & 4.7161 & 0.21204 \\
\hline 1153.8 & 2.5000 & 4.7092 & 0.21235 \\
\hline 1155.5 & 2.5000 & 4.7023 & 0.21266 \\
\hline 1157.1 & .5000 & 4 & 97 \\
\hline 1158.8 & .5000 & 4.6886 & 0.21328 \\
\hline 1160.4 & .5000 & 4.6818 & 0.21359 \\
\hline 1162.1 & .5000 & 4.6750 & 0.21390 \\
\hline 1163.8 & 2.5000 & 4.6682 & 0.21422 \\
\hline 11654 & .5000 & 4. & 453 \\
\hline 1167.1 & .5000 & 4.6547 & 21484 \\
\hline & .5000 & & \\
\hline 4 & 5000 & 4 & 46 \\
\hline 1172.1 & 2.5000 & 4.6346 & 1577 \\
\hline 1173.7 & .5000 & 4.6279 & 1608 \\
\hline 1175.4 & 2.5000 & 4.6213 & 21639 \\
\hline 1177.0 & .5000 & 4.6 & 570 \\
\hline & 5000 & & \\
\hline 1180.4 & 2.5000 & 4.6014 & 1732 \\
\hline 1182.0 & .5000 & & \\
\hline 1183.7 & 2.5000 & 4.5883 & 0.21794 \\
\hline 1185.3 & 2.5000 & 4.5818 & 21826 \\
\hline 1187.0 & .5000 & 4. & 857 \\
\hline 1188.7 & 2.5000 & 4.5688 & 21888 \\
\hline 1190.3 & .5000 & & 919 \\
\hline & & & \\
\hline 1193.6 & 2.5000 & 94 & 1981 \\
\hline 1195.3 & .5000 & 4.5430 & 0.22012 \\
\hline 1197.0 & 2.5000 & & 0.22043 \\
\hline 8.6 & 2.5000 & 02 & 2074 \\
\hline & & & \\
\hline 1201.9 & 2.5000 & 4.5175 & 0.22136 \\
\hline 1203.6 & 2.5000 & 4.5112 & 0.22167 \\
\hline & & & \\
\hline & & & \\
\hline
\end{tabular}




\begin{tabular}{|c|c|c|c|c|c|c|c|c|c|c|c|c|c|}
\hline & & & 260 & & & & & & & & & & \\
\hline 0.2 & 000 & 61 & 291 & 2.7 & & 643 & & & & & & & \\
\hline 1.9 & 5000 & 4798 & 22322 & 26.0 & 84.0 & 3676 & 9826 & 4559 & 0.48 & 3533 & $5576 e-05$ & 12816 & 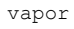 \\
\hline 3.6 & 000 & 4.4736 & 2353 & 3929.3 & 488.1 & .3710 & .9837 & 2.4569 & 831.02 & 1.3478 & 5 & 12840 & \\
\hline 15.2 & 2.5000 & 4.4674 & 22384 & 3932.6 & 492.2 & 8.3744 & .9848 & 2.4579 & 31.55 & 1.3422 & $5695 e-05$ & 12864 & \\
\hline & 5000 & & 2415 & 35.9 & 96.3 & & & & 08 & & & & \\
\hline & 5000 & & 2446 & 39.2 & 00.3 & 3811 & & 4600 & 832.61 & & & & \\
\hline 20.2 & .5000 & 4.4489 & .22477 & 3942.5 & 4504.4 & .3844 & 9880 & 2.4610 & 833.15 & 58 & & 2935 & rapor \\
\hline & & & & & 508.5 & & & & & & & & \\
\hline 23.5 & 2.5000 & 4. & 539 & .1 & 4512.6 & & & & & & & & \\
\hline 5.2 & .5000 & 306 & 0.22570 & 52.4 & 4516.7 & 8.3944 & 912 & 4640 & & 096 & & 006 & $\mathrm{apc}$ \\
\hline & & & & & & & & & & & & & \\
\hline 8.5 & 2.5000 & & 632 & & .9 & & & & & & & & P \\
\hline & 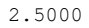 & & & & & & & & & & & & \\
\hline 8 & 2.5 & & & & .1 & & & & & & & & \\
\hline 3.5 & 2.5000 & & 0.22725 & .0 & 4537.2 & 8.4111 & & & & & & 125 & p \\
\hline & & & & & & & & & & & & & \\
\hline 5.8 & 2.5 & & & & & & & & & & & & \\
\hline & 00 & & 19 & & & & & & & & & & apor \\
\hline & 00 & & 850 & 3 & 3.6 & & & & & & & & $\mathrm{p}$ \\
\hline & 2.5 & & & & & & & & & & & & Tr \\
\hline .4 & 2.5000 & & 912 & 39.0 & 1.8 & 10 & & & & & & 68 & $\mathrm{p}$ \\
\hline 5.1 & 2.5000 & & 943 & 92.3 & & & & & & & & 292 & por \\
\hline & & & & & & & & & & & & & aps \\
\hline & 00 & & & & 4.1 & & & & & & & 40 & p \\
\hline & 25 & & & & & & & & & & & & pe \\
\hline & & & & & & & & & & & & & \\
\hline 3.4 & 2.5000 & & 098 & 4009.0 & 4586.5 & 8.4508 & 2.0093 & 13 & .70 & & & 3412 & a \\
\hline & & & & & & & & & & & & & \\
\hline & 2.5 & & & & .7 & & & & & & & & p \\
\hline & 2.5 & & & & & & & & & & & & $\mathrm{p}$ \\
\hline & & & & & & & & & & & & & \\
\hline 1.7 & 2.5000 & 4.3 & 252 & 4025.8 & 4607.1 & 8.4671 & 45 & 54 & 31 & 77 & & 531 & 年 \\
\hline & & & & & & & & & & & & & \\
\hline & & & & & & & & & & & & 579 & \\
\hline & 2.5000 & & 45 & 5.8 & & & & & & & & 503 & s \\
\hline & & & & & & & & & & & & & \\
\hline 0.0 & 00 & 4.2722 & 0.23407 & 042.6 & 4627.7 & 8.4835 & 2.0198 & 2.4 & 848.92 & 1. & $39 e-05$ & 0.13651 & \\
\hline
\end{tabular}




\section{REFERENCES}

[1] 2017. “ANS Nuclear Grand Challenges.” Special Report, American Nuclear Society. https://www.ans.org/challenges/.

[2] GenIV International Forum. 2014. "Technology Roadmap Update for Generation IV Nuclear Energy Systems.” OECD Nuclear Energy Agency for the Generation IV International Forum.

[3] 2020. "Overview of energy markets." U.S. Energy Information Administration. https://www.eia.gov/outlooks/aeo/pdf/AEO2020\%20Overview\%20of\%20Energy\%20Markets.pdf.

[4] 2020. "NRC Non-Light Water Reactor (Non-LWR) Vision and Strategy, Volume 1 - Computer Code Suite for Non-LWR Plant Systems Analysis.” Rev.1, U.S. Nuclear Regulatory Commission.

[5] Beausoleil II, G.L., G.L. Povirk, and B.J. Curnutt. 2020. "A Revised Capsule Design for the Accelerated Testing of Advanced Reactor Fuels.” Nuclear Technology, 206: 444-457.

[6] 2018. "Bounding Thermal-Hydraulic Analysis for ATF Fuels Irradiation Program.” ECAR-2298, Rev. 3, Project 28203, Idaho National Laboratory.

[7] Crawford, D. C., D. L. Porter, S. L. Hayes, M. K. Meyer, D. A. Petti, and K. Pasamehmetoglu. 2007. “An Approach to Fuel Development and Qualification.” Journal of Nuclear Materials 371: 232-242.

[8] Terrani, K. A. 2018. “Accident Tolerant Fuel Cladding Development: Promise, Status, and Challenges." Journal of Nuclear Materials 501: 13-30.

[9] S. S.97 - 115th Congress (2017-2018): Nuclear Energy Innovation Capabilities Act of 2017 | Congress.gov | Library of Congress. https://www.congress.gov/bill/115th congress/senate-bill/97.

[10] Aguiar, J.A., M. Kerr, R. Kennedy, S. Hayes, and R.A. Roach. 2020. "Nuclear Materials Discovery and Qualification Initiative." INL/EXT-20-57732, Rev.0, Idaho National Laboratory.

[11] Aguiar, J.A., A.M. Jokisaari, M. Kerr, and R.A. Roach. 2020. "Bringing Nuclear Materials Discovery and Qualification into the $21^{\text {st }}$ Century." Nature Communications, 11(2556): 1-3.

[12] 2009. “Advanced Test Reactor National Scientific User Facility User's Guide.” INL/EXT-08-14709, Idaho National Laboratory.

[13] Smith, M. 2009. ABAQUS/Standard User's Manual, Version 6.9. Dassault Systèmes Simulia Corp, Providence, RI.

[14] Beausoleil II, G.L., B.J. Curnutt, and G.L. Povirk. 2018. “A Revised Capsule Design for the Accelerated Testing of Advanced Reactor Fuels." INL/EXT-18-45933, Rev.0, Idaho National Laboratory.

[15] Nuclear Regulatory Commission. 1995. "RELAP5/MOD3 Code Manual, Volume I: Code Structure, System Models and Solution Methods.” NUREG/CR-5535, Nuclear Regulatory Commission.

[16] Nuclear Regulatory Commission. 2007. “TRACE V5.0 Theory Manual.” Nuclear Regulatory Commission.

[17] 2017. "MELCOR Computer Code Manuals, Vol. 2: Reference Manual, Version 2.2.9541," SAND 2017-0876 O (ADAMS Accession No. ML17040A420), Sandia National Laboratories.

[18] Kaufman, N., J. Tappendorf, J. Durney, and J. Willis. 1969. "Reactor Physics Reuslts from LowPower Measurements in the Advanced Test Reactor." IN-1260, Idaho Nuclear Corporation.

[19] Lemmon, Eric W., Mark O. McLinden, and Daniel G. Friend. 2018. "Thermophysical Properties of Fluid Systems" in the NIST Chemistry WebBook, NIST Standard Reference Database Number 69, 
Edited by P.J. Linstrom and W.G. Mallard, National Institute of Standards and Technology, Gaithersburg MD, 20899, https://doi.org/10.18434/T4D303.

[20] Dittus, F.W. and L.M.K. Boelter. 1930. "Heat Transfer in Automobile Radiators of the Tubular Type." University of California Publications in Engineering, 2: 443-461.

[21] Clément, S.A. and P.M. Bardet. 2017. "Surrogates for Single-Phase Conjugate Heat Transfer Validation Experiments at Light Water Reactor Prototypical Conditions.” Nuclear Technology, 199: 151-173.

[22] Sieder, E.N. and G.E. Tate. 1936. "Heat Transfer and Pressure Drop of Liquids in Tubes." Industrial and Engineering Chemistry, 28(12): 1429-1435.

[23] Gnielinski, V. 1975. "New Equations for Heat and Mass Transfer in Pipes and Ducts with a Turbulent Flow." Engineering Research A (Translated from German), 41: 8-16.

[24] 2019. “Thermal-Hydraulic Analysis Team (THAT) Guidebook.” GDE-588, Rev. 3, Idaho National Laboratory.

[25] White, F. 2009. "Fluid Mechanics." McGraw-Hill Series in Mechanical Engineering, $7^{\text {th }}$ Ed: p.371.

[26] ASME Boiler and Pressure Vessel Code, Sec. VIII, Div 1 (UG-27), 2019.

[27] Bernath, L. 1960. "A Theory of Local-Boiling Burnout and Its Application to Existing Data." Chemical Engineering Progress Symposium Series, 56(30): 95-116.

[28] Walker, V.A. 1960. "Transmittal of Burnout Survey.” United States Government Memorandum. https:/www.nrc.gov/docs/ML0217/ML021780287.pdf.

[29] Beitel, G.R. 1993. "Boiling Heat-Transfer Processes and Their Application in the Cooling of High Heat Flux Devices.” AEDC-TR-93-3, OMB 0704-0188. https://apps.dtic.mil/dtic/tr/fulltext/u2/a266086.pdf.

[30] Todreas, N.E. and M.S. Kazimi. 2011. Nuclear Systems Volume 1: Thermal Hydraulic Fundamentals. CRC Press: Taylor \& Francis Group, $2^{\text {nd }}$ Ed, Revised Printing.

[31] Çengel, Y.A. and M.A. Boles. Thermodynamics: An Engineering Approach. $8^{\text {th }}$ Ed., McGraw-Hill Education.

[32] 2019. “AFC Fission Accelerated Steady State Testing Experiment Temperature Analysis.” ECAR4968, Rev. 0, Project 28203, Idaho National Laboratory.

[33] Billone, M.C., Y.Y. Liu, E.E. Gruber, T.H. Hughes, and M.J. Kramer. 1986. "Status of Fuel Element Modeling Codes for Metallic Fuels." Proceedings of the American Nuclear Society Conference on Reliable Fuels for Liquid Metal Reactors, September 7-11, 1986: 5-77.

[34] IFR Property Evaluation Working Group. 1988. "Metallic Fuels Handbook.” ANL-NSE-3, Rev.1, Argonne National Laboratory.

[35] Umezawa, O. and K. Ishikawa. 1992. "Electrical and Thermal Conductivities and Magnetization of Some Austenitic Steels, Titanium and Titanium Alloys at Cryogenic Temperatures." Cryogenics, 32(10): 873-880.

[36] Lucks, C.F., H.B. Thimpson, A.R. Smith, F.P. Curry, H.W. Deem, and G.F. Bing. 1951. "United States Air Force Technical Report.” USAF-TR-6145-1, United States Air Force.

[37] Siefken, L.J., E.W. Coryell, E.A. Harvego, and J.K. Hohorst. 2001. "SCDAP/RELAP5/MOD 3.3 Code Manual: MATPRO - A Library of materials Properties for Light-Water-Reactor Accident Analysis.” NUREG/CR-6150, Vol. 4, Rev. 2, Nuclear Regulatory Guide. 
[38] Ho, C.Y., R.W. Powell, and P.E. Liley. 1972. "Thermal Conductivity of the Elements.” Journal of Physical and Chemical Reference Data, 1: 279-421.

[39] Newman, C., G. Hansen, and D. Gaston. 2009. "Three Dimensional Coupled Simulation of Thermomechanics, Heat, and Oxygen Diffusion in UO2 Nuclear Fuel Rods.” Journal of Nuclear Materials, 392(1): 6-15.

[40] Gaston, D., C. Newman, G. Hansen, D. Lebrun-Grandié. 2009. "MOOSE: A Parallel Computational Framework for Coupled Systems of Nonlinear Equations." Nuclear Engineering and Design 239: $1768-1778$. 